\title{
From Statistical Knowledge Bases to Degrees of Belief*
}

\author{
Fahiem Bacchus \\ Computer Science Dept. \\ University of Waterloo \\ Waterloo, Ontario \\ Canada, N2L 3G1 \\ fbacchus@logos.uwaterloo.ca \\ Joseph Y. Halpern \\ IBM Almaden Research Center \\ 650 Harry Road \\ San Jose, CA 95120-6099 \\ halpern@almaden.ibm.com
}

\author{
Adam J. Grove \\ NEC Research Institute \\ 4 Independence Way \\ Princeton, NJ 08540 \\ grove@research.nj.nec.com
}

\author{
Daphne Koller \\ Computer Science Department \\ Stanford University \\ Stanford, CA 94305 \\ koller@cs.stanford.edu
}

\begin{abstract}
An intelligent agent will often be uncertain about various properties of its environment, and when acting in that environment it will frequently need to quantify its uncertainty. For example, if the agent wishes to employ the expected-utility paradigm of decision theory to guide its actions, it will need to assign degrees of belief (subjective probabilities) to various assertions. Of course, these degrees of belief should not be arbitrary, but rather should be based on the information available to the agent. This paper describes one approach for inducing degrees of belief from very rich knowledge bases, that can include information about particular individuals, statistical correlations, physical laws, and default rules. We call our approach the random-worlds method. The method is based on the principle of indifference: it treats all of the worlds the agent considers possible as being equally likely. It is able to integrate qualitative default reasoning with quantitative probabilistic reasoning by providing a language in which both types of information can be easily expressed. Our results show that a number of desiderata that arise in direct inference (reasoning from statistical information to conclusions about individuals) and default reasoning follow directly from the semantics of random worlds. For example, random worlds captures important patterns of reasoning such as specificity, inheritance, indifference to irrelevant information, and default assumptions of independence. Furthermore, the expressive power of the language used and the intuitive semantics of random worlds allow the method to deal with problems that are beyond the scope of many other non-deductive reasoning systems.
\end{abstract}

\footnotetext{
${ }^{*}$ A preliminary version of this paper appeared in the International Joint Conference on Artificial Intelligence, 1993 [BGHK93]. This version is essentially identical to one that appears in Artificial Intelligence 87:1-2, 1996, pp. 75-143. Some of this work was performed while Adam Grove was at Stanford University and at IBM Almaden Research Center, and while Daphne Koller was at U.C. Berkeley and at IBM Almaden Research Center. This research has been supported in part by the Canadian Government through their NSERC and IRIS programs, by the Air Force Office of Scientific Research (AFOSR) under Contract F49620-91-C-0080, by an IBM Graduate Fellowship, and by a University of California President's Postdoctoral Fellowship. The United States Government is authorized to reproduce and distribute reprints for governmental purposes.
} 


\section{Introduction}

Consider an agent with a knowledge base, $K B$, who has to make decisions about its actions in the world. For example, a doctor may need to decide on a treatment for a particular patient, say Eric. The doctor's knowledge base might contain information of different types, including: statistical information, e.g., "80\% of patients with jaundice have hepatitis"; firstorder information, e.g., "all patients with hepatitis have jaundice"; default information, e.g., "patients with hepatitis typically have a fever"; and information about the particular patient at hand, e.g., "Eric has jaundice". In most cases, the knowledge base will not contain complete information about a particular individual. For example, the doctor may be uncertain about the exact disease that Eric has. Since the efficacy of a treatment will almost certainly depend on the disease, it is important for the doctor to be able to quantify the relative likelihood of various possibilities. More generally, to apply standard tools for decision making such as decision theory (see, e.g., [LR57, Sav54]), an agent must assign probabilities, or degrees of belief, to various events. For example, the doctor may wish to assign a degree of belief to an event such as "Eric has hepatitis". This paper describes one particular method that allows such an agent to use its knowledge base to assign degrees of belief in a principled manner; we call this method the random-worlds method.

There has been a great deal of work addressing aspects of this general problem. Two large bodies of work that are particularly relevant are the work on direct inference, going back to Reichenbach [Rei49], and the various approaches to nonmonotonic reasoning. Direct inference deals with the problem of deriving degrees of belief from statistical information, typically by attempting to find a suitable reference class whose statistics can be used to determine the degree of belief. For instance, a suitable reference class for the patient Eric might be the class of all patients with jaundice. While direct inference is concerned with statistical knowledge, the field of nonmonotonic reasoning, on the other hand, deals mostly with knowledge bases that contain default rules. As we shall argue, none of the systems proposed for either referenceclass reasoning or nonmonotonic reasoning can deal adequately with the large and complex knowledge bases we are interested in. In particular, none can handle rich knowledge bases that may contain first-order, default, and statistical information. The random-worlds approach, on the other hand, can deal with such complex knowledge bases, and handles several paradigmatic problems in both nonmonotonic and reference-class reasoning remarkably well.

We now provide a brief overview of the random-worlds approach. We assume that the information in the knowledge base is expressed in a variant of the language introduced by Bacchus [Bac90]. Bacchus's language augments first-order logic by allowing statements of the form $\|\operatorname{Hep}(x) \mid \operatorname{Jaun}(x)\|_{x}=0.8$, which says that $80 \%$ of patients with jaundice have hepatitis. Notice, however, that in finite models this statement has the (probably unintended) consequence that the number of patients with jaundice is a multiple of 5 . To avoid this problem, we use approximate equality rather than equality, writing $\|H e p(x) \mid \operatorname{Jaun}(x)\|_{x} \approx 0.8$, read "approximately $80 \%$ of patients with jaundice have hepatitis". Intuitively, this says that the proportion of jaundiced patients with hepatitis is close to $80 \%$ : i.e., within some tolerance $\tau$ of 0.8 .

Not only does the use of approximate equality solve the problem of unintended consequences, it has another significant advantage: it lets us express default information. We interpret a statement such as "Birds typically fly" as expressing the statistical assertion that "Almost 
all birds fly". Using approximate equality, we can represent this as $\|F l y(x) \mid \operatorname{Bird}(x)\|_{x} \approx 1$. This interpretation is closely related to various approaches applying probabilistic semantics to nonmonotonic logic; see Pearl [Pea89] for an overview of these approaches, and Section 6 for further discussion.

Having described the language in which our knowledge base is expressed, we now need to decide how to assign degrees of belief given a knowledge base. Perhaps the most widely used framework for assigning degrees of belief (which are essentially subjective probabilities) is the Bayesian paradigm. There, one assumes a space of possibilities and a probability distribution over this space (the prior distribution), and calculates posterior probabilities by conditioning on what is known (in our case, the knowledge base). To use this approach, we must specify the space of possibilities and the distribution over it. In Bayesian reasoning, there is relatively little consensus as to how this should be done in general. Indeed, the usual philosophy is that these decisions are subjective. The difficulty of making these decisions seems to have been an important reason for the historic unpopularity of the Bayesian approach in symbolic AI [MH69].

Our approach is different. We assume that the $K B$ contains all the knowledge the agent has, and we allow a very expressive language so as to make this assumption reasonable. This assumption means that any knowledge the agent has that could influence the prior distribution is already included in the $K B$. As a consequence, we give a single uniform construction of a space of possibilities and a distribution over it. Once we have this probability space, we can use the Bayesian approach: To compute the probability of an assertion $\varphi$ given $K B$, we condition on $K B$, and then compute the probability of $\varphi$ using the resulting posterior distribution.

So how do we choose the probability space? One general strategy, discussed by Halpern [Hal90], is to give semantics to degrees of belief in terms of a probability distribution over a set of possible worlds, or first-order models. This semantics clarifies the distinction between statistical assertions and degrees of belief. As we suggested above, a statistical assertion such as $\|\operatorname{Hep}(x) \mid \operatorname{Jaun}(x)\|_{x} \approx 0.8$ is true or false in a particular world, depending on how many jaundiced patients have hepatitis in that world. On the other hand, a degree of belief is neither true nor false in a particular world - it has semantics only with respect to the entire set of possible worlds and a probability distribution over them. There is no necessary connection between the information in the agent's $K B$ and the distribution over worlds that determines her degrees of belief. However, we clearly want there to be some connection. In particular, we want the agent to base her degrees of beliefs on her information about the world, including her statistical information. As this paper shows, the random-worlds method is a powerful technique for accomplishing this.

To define our probability space, we have to choose an appropriate set of possible worlds. Given some domain of individuals, we stipulate that the set of worlds is simply the set of all first-order models over this domain. That is, a possible world corresponds to a particular way of interpreting the symbols in the agent's vocabulary over the domain. In our context, we can assume that the "true world" has a finite domain, say of size $N$. In fact, without loss of generality, we assume that the domain is $\{1, \ldots, N\}$.

Having defined the probability space (the set of possible worlds), we must construct a probability distribution over this set. For this, we give perhaps the simplest possible definition: we assume that all the possible worlds are equally likely (that is, each world has the same probability). This can be viewed as an application of the principle of indifference. Since 
we are assuming that all the agent knows is incorporated in her knowledge base, the agent has no a priori reason to prefer one world over the other. It is therefore reasonable to view all worlds as equally likely. Interestingly, the principle of indifference (sometimes also called the principle of insufficient reason) was originally promoted as part of the very definition of probability when the field was originally formalized by Jacob Bernoulli and others; the principle was later popularized further and applied with considerable success by Laplace. (See [Hac75] for a historical discussion.) It later fell into disrepute as a general definition of probability, largely because of the existence of paradoxes that arise when the principle is applied to infinite or continuous probability spaces. We claim, however, that the principle of indifference can be a natural and effective way of assigning degrees of belief in certain contexts, and in particular, in the context where we restrict our attention to a finite collection of worlds.

Combining our choice of possible worlds with the principle of indifference, we obtain our prior distribution. We can now induce a degree of belief in $\varphi$ given $K B$ by conditioning on $K B$ to obtain a posterior distribution and then computing the probability of $\varphi$ according to this new distribution. It is easy to see that, since each world is equally likely, the degree of belief in $\varphi$ given $K B$ is the fraction of possible worlds satisfying $K B$ that also satisfy $\varphi$.

One problem with the approach as stated so far is that, in general, we do not know the domain size $N$. Typically, however, $N$ is known to be large. We therefore approximate the degree of belief for the true but unknown $N$ by computing the limiting value of this degree of belief as $N$ grows large. The result is our random-worlds method.

The key ideas in the approach are not new. Many of them can be found in the work of Johnson [Joh32] and Carnap [Car50, Car52], although these authors focus on knowledge bases that contain only first-order information, and for the most part restrict their attention to unary predicates. Related approaches have been used in the more recent works of Shastri [Sha89] and of Paris and Vencovska [PV89], in the context of a unary statistical language. Chuaqui's recent work [Chu91] is also relevant. His work, although technically quite different from ours, shares the idea of basing a theory of probabilistic reasoning upon notions of indifference and symmetry. The works of Chuaqui and Carnap investigate very different issues from those we examine in this paper. For example, Carnap, and others who later continued to develop his ideas, were very much interested in inductive learning (especially the problem of learning universal laws). While we believe the question of learning is very important (see Section 7.3), we have largely concentrated on understanding (and generalizing) the process of going from statistical information and default rules to inferences about particular individuals. Many of the new results we describe reflect this different emphasis.

Having defined the method, how do we judge its reasonableness? Fortunately, as we mentioned, there are two large bodies of work on related problems from which we can draw guidance: reference-class reasoning and default reasoning. While none of the solutions suggested for these problems seems entirely adequate, the years of research have resulted in some strong intuitions regarding what answers are intuitively reasonable for certain types of queries. Interestingly, these intuitions often lead to identical desiderata. In particular, most systems (of both types) espouse some form of preference for more specific information and the ability to ignore irrelevant information. We show that the random-worlds approach satisfies these desiderata. In fact, in the case of random worlds, these properties follow from two much general theorems. We prove that, in those cases where there is a specific piece of statistical information that should "obvi- 
ously" be used to determine a degree of belief, random worlds does in fact use this information. The different desiderata, such as a preference for more specific information and an indifference to irrelevant information follow as easy corollaries. We also show that random worlds provides reasonable answers in many other contexts, not covered by the standard specificity and irrelevance heuristics. Thus, the random-worlds method is indeed a powerful one, that can deal with rich knowledge bases and still produce the answers that people have identified as being the most appropriate ones.

The rest of this paper is organized as follows. In the next two sections, we outline some of the major themes and problems in the work on reference classes and on default reasoning. Since one of our major claims is that the random-worlds approach solves many of these problems, this will help set our work in context. In Section 4, we describe the random-worlds method in detail. In Section 5, we state and prove a number of general theorems about the properties of the approach, and show how various desiderata follow from these theorems. In Section 6 we discuss the problem of calculating degrees of belief. Using results from [GHK94], we demonstrate a close connection between random worlds and maximum entropy in the case of unary knowledge bases. Based on this connection, we show that in many cases of interest a maximum-entropy computation can be used to calculate an agent's degree of belief. Furthermore, we show that the maximum-entropy approach to default reasoning considered in [GMP90] can be embedded in our framework. Finally, we discuss some possible criticisms and limitations of the randomworlds method in Section 7 and the possible impact of the method in Section 8.

\section{Reference classes}

Strictly speaking, the only necessary relationship between objective knowledge about frequencies and proportions on the one hand and degrees of belief on the other hand is the simple mathematical fact that they both obey the axioms of probability. But in practice we usually hope for a deeper connection: the latter should be based on the former in some "reasonable" way. Of course, the random-worlds approach that we are advocating is precisely a theory of how this connection can be made. But our approach is far from the first to attempt to connect statistical information and degrees of belief. Most of the earlier work is based on the idea of finding a suitable reference class. In this section, we review some of this work and show why we believe that this approach, while it has some intuitively reasonable properties, is inadequate as a general methodology. (See also [BGHK94c] for further discussion of this issue.) We go into some detail here, since the issues that arise provide some motivation for the results that we prove later regarding our approach.

\subsection{The basic approach}

The earliest sophisticated attempt at clarifying the connection between objective statistical knowledge and degrees of belief, and the basis for most subsequent proposals, is due to Reichenbach [Rei49]. Reichenbach describes the idea as follows:

"If we are asked to find the probability holding for an individual future event, we must first incorporate the case in a suitable reference class. An individual thing 
or event may be incorporated in many reference classes... . We then proceed by considering the narrowest [smallest] reference class for which suitable statistics can be compiled."

Although not stated explicitly in this quote, Reichenbach's approach was to equate the degree of belief in the individual event with the statistics from the chosen reference class. As an example, suppose that we want to determine a probability (i.e., a degree of belief) that Eric, a particular patient with jaundice, has the disease hepatitis. The particular individual Eric is a member of the class of all patients with jaundice. Hence, following Reichenbach, we can use the class of all such patients as a reference class, and assign a degree of belief equal to our statistics concerning the frequency of hepatitis among this class. If we know that this frequency is $80 \%$, then we would assign a degree of belief of 0.8 to the assertion that Eric has hepatitis.

Reichenbach's approach consists of (1) the postulate that we use the statistics from a particular reference class to infer a degree of belief with the same numerical value, and (2) some guidance as to how to choose this reference class from a number of competing reference classes. We consider each point in turn.

In general, a reference class is simply a set of domain individuals ${ }^{1}$ that contains the particular individual about whom we wish to reason and for which we have "suitable statistics". In our framework, we may take the set of individuals satisfying a formula $\psi(x)$ to be a reference class. The requirement that the particular individual $c$ we wish to reason about belongs to the class is then represented by the logical assertion $\psi(c) .^{2}$ But what does the phrase "suitable statistics" mean? Suppose for now we take a "suitable statistic" to be a closed interval that is nontrivial, i.e., that is not $[0,1]$, in which the proportion or frequency lies. More precisely, consider some query $\varphi(c)$, where $\varphi$ is some logical assertion and $c$ is a constant, denoting some individual in the domain. Then, under this interpretation, $\psi(x)$ is a reference class for this query if we know both $\psi(c)$ and $\|\varphi(x) \mid \psi(x)\|_{x} \in[\alpha, \beta]$, for some nontrivial interval $[\alpha, \beta]$. That is, we know that $c$ has property $\psi$, and that among the class of individuals that possess property $\psi$, the proportion that also have property $\varphi$ is between $\alpha$ and $\beta$. If we decide that this is the appropriate reference class then, using Reichenbach's approach, we would conclude $\operatorname{Pr}(\varphi(c)) \in[\alpha, \beta]$, i.e., the probability (degree of belief) that $c$ has property $\varphi$ is between $\alpha$ and $\beta$. Note that the appropriate reference class for the query $\varphi(c)$ depends both on the formula $\varphi(x)$ and on the individual $c$.

Given a query $\varphi(c)$, there will in general be many reference classes that are arguably appropriate for it. For example, suppose we know both $\psi_{1}(c)$ and $\psi_{2}(c)$, and we have two pieces of statistical information: $\left\|\varphi(x) \mid \psi_{1}(x)\right\|_{x} \in\left[\alpha_{1}, \beta_{1}\right]$ and $\left\|\varphi(x) \mid \psi_{2}(x)\right\|_{x} \in\left[\alpha_{2}, \beta_{2}\right]$. In this case both $\psi_{1}(x)$ and $\psi_{2}(x)$ are reference classes for $\varphi(c)$ and, depending on the values of the $\alpha$ 's and $\beta$ 's, they could assign conflicting degrees of belief to $\varphi(c)$. The second part of Reichenbach's approach is intended to deal with the problem of how to choose a single reference class from a set of possible classes. Reichenbach recommended preferring the narrowest (i.e., the smallest,

\footnotetext{
${ }^{1}$ These "individuals" might be complex objects (such as sequences of coin tosses) depending on what we take as primitive in our ontology.

${ }^{2}$ Although the examples in this section deal with reasoning about single individuals, in general both referenceclass reasoning and random worlds can be applied to queries such as "Did Eric infect Tom", which involve reasoning about a number of individuals simultaneously. In such cases the reference classes will consist of sets of tuples of individuals.
} 
or most specific) class. In this example, if we know $\forall x\left(\psi_{1}(x) \Rightarrow \psi_{2}(x)\right)$, so that the class $\psi_{1}(x)$ is a subset of the class $\psi_{2}(x)$, then, using Reichenbach's approach, we would take the statistics from the more specific reference class $\psi_{1}(x)$ and conclude that $\operatorname{Pr}(\varphi(c)) \in\left[\alpha_{1}, \beta_{1}\right]$.

These two parts of Reichenbach's approach - using statistics taken from a class as a degree of belief about an individual and preferring statistics from more specific classes - are generally reasonable and intuitively compelling when applied to simple examples. Of course, even on the simplest examples Reichenbach's strategy cannot be said to be "correct" in any absolute sense. Nevertheless, it is impressive that there is such widespread agreement as to the reasonableness of the answers. As we show later, the random-worlds approach agrees with both aspects of Reichenbach's approach when applied to simple (and uncontroversial) examples. Unlike that approach, however, the random-worlds approach derives these intuitive answers from more basic principles. As a result, it is able to deal well with more complex examples that defeat Reichenbach's approach.

Despite its successes, Reichenbach's approach has several serious problems. For one thing, defining what counts as a "suitable statistic" is not easy. For another, it is clear that the principle of preferring more specific information rarely suffices to deal with the cases that arise with a rich knowledge base. Nevertheless, much of the work on connecting statistical information and degrees of belief, including that of Kyburg [Kyb83, Kyb74] and of Pollock [Pol90], has built on Reichenbach's ideas of reference classes by elaborating the manner in which choices are made between reference classes. As a result, these later approaches all suffer from a similar set of difficulties, which we now discuss.

\subsection{Identifying reference classes}

Recall that we took a reference class to be simply a set for which we have "suitable statistics". But if any set of individuals whatsoever can potentially serve as a reference class then problems arise. Assume we know $\operatorname{Jaun}(\operatorname{Eric})$ and $\|\operatorname{Hep}(x) \mid \operatorname{Jaun}(x)\|_{x} \approx 0.8$. In this case $\operatorname{Jaun}(x)$ is a legitimate reference class for the query $H e p(E r i c)$. Therefore, we would like to conclude that $\operatorname{Pr}(H e p(E r i c))=0.8$. But Eric is also a member of the more specific class of jaundiced patients without hepatitis together with $\{$ Eric $\}$ (i.e., the class defined by the formula $(\operatorname{Jaun}(x) \wedge$ $\neg H e p(x)) \vee x=E r i c)$. If there are quite a few jaundiced patients without hepatitis, then we have excellent statistics for the proportion of patients in this class with hepatitis: it is approximately $0 \%$. Thus, the conclusion that $\operatorname{Pr}(\operatorname{Hep}($ Eric $))=0.8$ is disallowed by the rule instructing us to use the most specific reference class. In fact, it seems that we can almost always find a more specific class that will give a different and intuitively incorrect answer. This example suggests that we cannot take an arbitrary set of individuals to be a reference class; it must satisfy additional criteria.

Kyburg and Pollock deal with this difficulty by placing restrictions on the set of allowable reference classes that, although different, have the effect of disallowing disjunctive reference classes, including the problematic class described above. This approach suffers from two deficiencies. First, as Kyburg himself has observed [Kyb74], these restrictions do not eliminate the problem completely. Furthermore, restricting the set of allowable reference classes may prevent us from making full use of the information we have. For example, the genetically inherited disease Tay-Sachs (represented by the predicate $T S$ ) appears only in babies of two distinct 
populations: Jews of east-European extraction $(E E J)$, and French-Canadians from a certain geographic area $(F C)$. Within the afflicted population, Tay-Sachs occurs in $2 \%$ of the babies. The agent might represent this fact using the statement $\|T S(x) \mid E E J(x) \vee F C(x)\|_{x}=0.02$. However, if disjunctive reference classes are disallowed, then the agent would not be able to use this information in reasoning.

It is clear that if one takes the reference-class approach to generating degrees of belief, some restrictions on what constitutes a legitimate reference class are inevitable. Unfortunately, it seems that the current approaches to this problem are inadequate. The random-worlds approach does not depend on the notion of a reference class, and so is not forced to confront this issue.

\subsection{Competing reference classes}

Even if the problem of defining the set of "legitimate" reference classes can be resolved, the reference-class approach must still address the problem of choosing the "right" class out of the set of legitimate ones. The solution to this problem has typically been to posit a collection of rules indicating when one reference class should be preferred over another. The basic criterion is the one we already mentioned: choose the most specific class. But even in the cases to which this specificity rule applies, it is not always appropriate. Assume, for example, that we know that between $70 \%$ and $80 \%$ of birds chirp and that between $0 \%$ and $99 \%$ of magpies chirp. If Tweety is a magpie, the specificity rule would tell us to use the more specific reference class, and conclude that $\operatorname{Pr}($ Chirps $($ Tweety $)) \in[0,0.99]$. Although the interval [0,0.99] is certainly not trivial, it is not very meaningful. Had the 0.99 been a 1 , the interval would have been trivial, and we could have then ignored this class and used the more detailed statistics of $[0.7,0.8]$ derived from the class of birds.

The knowledge base above might be appropriate for someone who knows little about magpies, and so feels less confidence in his statistics for magpies than in his statistics for the class of birds as a whole. But since $[0.7,0.8] \subseteq[0,0.99]$, we know nothing that indicates that magpies are actually different from birds in general with respect to chirping. There is an alternative intuition that says that if the statistics for the less specific reference class (the class of birds) are more precise, and they do not contradict the statistics for the more specific class (magpies), then we should use them. That is, we should conclude that $\operatorname{Pr}($ Chirps $($ Tweety $)) \in[0.7,0.8]$. This intuition is captured and generalized in Kyburg's strength rule.

Unfortunately, neither the specificity rule nor its extension by Kyburg's strength rule are adequate in most cases. In typical examples, the agent generally has several incomparable classes relevant to the problem, so that neither rule applies. Reference-class systems such as Kyburg's and Pollock's simply give no useful answer in these cases. For example, suppose we know that Fred has high cholesterol and is a heavy smoker, and that $15 \%$ of people with high cholesterol get heart disease. If this is the only suitable reference class, then (according to all the systems $) \operatorname{Pr}($ Heart-disease $($ Fred $))=0.15$. On the other hand, suppose we then acquire the additional information that $9 \%$ of heavy smokers develop heart disease (but still have no nontrivial statistical information about the class of people with both attributes). In this case, neither class is the single right reference class, so approaches that rely on finding a single reference class generate a trivial range for the degree of belief that Fred will contract heart disease in this case. For example, Kyburg's system will generate the interval $[0,1]$ for the degree of belief. 
Giving up completely in the face of conflicting evidence seems to us to be inappropriate. The entire enterprise of generating degrees of belief is geared to providing the agent with some guidance for its actions (in the form of degrees of belief) when deduction is insufficient to provide a definite answer. That is, the aim is to generate plausible inferences. The presence of conflicting information does not mean that the agent no longer needs guidance. When we have several competing reference classes, none of which dominates the others according to specificity or any other rule that has been proposed, then the degree of belief should most reasonably be some combination of the corresponding statistical values. As we show later, the random-worlds approach does indeed combine the values from conflicting reference classes in a reasonable way, giving well-motivated answers even when the reference-class approach would fail.

\subsection{Other types of information}

We have already pointed out the problems that arise with the reference-class approach if more than one reference class bears on a particular problem. A more subtle problem is encountered in cases where there is relevant information that is not in the form of a reference class. We have said that for $\psi(x)$ to be a reference class for a query about $\varphi(c)$ we must know $\psi(c)$ and have some statistical information about $\|\varphi(x) \mid \psi(x)\|_{x}$. However, it is not sufficient to consider only the query $\varphi(c)$. Suppose we also know $\varphi(c) \Leftrightarrow \sigma(c)$ for some other formula $\sigma$. Then we would want $\operatorname{Pr}(\varphi(c))=\operatorname{Pr}(\sigma(c))$. But this implies that all of the reference classes for $\sigma(c)$ are relevant as well, because anything we can infer about $\operatorname{Pr}(\sigma(c))$ tells us something about $\operatorname{Pr}(\varphi(c))$. Both Pollock [Pol90] and Kyburg [Kyb83] deal with this by considering all of the reference classes for any formula $\sigma$ such that $\sigma(c) \Leftrightarrow \varphi(c)$ is known. However, they do not consider the case where it is known that $\sigma(c) \Rightarrow \varphi(c)$, which implies that $\operatorname{Pr}(\sigma(c)) \leq \operatorname{Pr}(\varphi(c))$, nor the case where it is known that $\varphi(c) \Rightarrow \sigma(c)$, which implies that $\operatorname{Pr}(\sigma(c)) \geq \operatorname{Pr}(\varphi(c))$. Thus, if we have a rich theory about $\varphi(c)$ and its implications, it can become very hard to locate all of the possible reference classes or even to define what qualifies as a possible reference class.

\subsection{Discussion}

A comparison between random worlds and reference-class approaches can be made in terms of the use of local versus global information. The reference-class approach is predicated on the assumption that we can always focus on a single piece of information, the statistics over a single reference class, that summarizes all the relevant information in the knowledge base. A strategy based on identifying a single relevant ("local") datum can offer great efficiency, but of course we should not expect this to be a general substitute for the use of all the ("global") information we have available. In this sense, the difficulties encountered by the reference-class approach are not surprising. When generating degrees of belief from a rich knowledge base, it will not always be possible to find a single reference class that captures all of the relevant information.

It is important to remember that although the notion of a reference class seems intuitive, it arises as part of one proposed solution strategy for the problem of computing degrees of belief. The notion of a reference classes is not part of the description of the problem, and there is no reason for it to necessarily be part of the solution. Indeed, as we have tried to argue, making it part of the solution can lead to more problems than it solves. 
Our approach makes no attempt to locate a single local piece of information (a reference class). Thus, all of the problems described above that arise from trying locate the "right" reference class vanish. Rather, it uses a semantic construction that takes into account all of the information in the knowledge base in a uniform manner. As we shall see, the random-worlds approach generates answers that agree with the reference-class approach in those special cases where there is a single appropriate reference class. However, it continues to give reasonable answers in many situations where no single local piece of information suffices. Furthermore, these answers are obtained directly from the simple semantics of random worlds, with no ad hoc rules and assumptions.

\section{Default reasoning}

One main claim of this paper is that the random-worlds method of inference, coupled with our statistical interpretation of defaults, provides a well-motivated and successful system of default reasoning. Evaluating such a claim is hard because there are many, sometimes rather vague, criteria for success that one can consider. In particular, not all criteria are appropriate for all default reasoning systems: Different applications (such as some of the ones outlined in [McC86]) require different interpretations for a default rule, and therefore need to satisfy different desiderata. Nevertheless, there are certain desiderata that have gained acceptance as measures for the success of a new nonmonotonic reasoning system. Some are general properties of nonmonotonic inference (see Section 3.2). Most, on the other hand, involve getting the "right" answers to a small set of standard examples (more often than not involving a bird called "Tweety"). As we claim at the end of this section, this has made an "objective" validation of proposed systems difficult, to say the least. In this section, we survey some of the desired properties for default reasoning and the associated problems and issues. Of course, our survey cannot be comprehensive. The areas we consider are the semantics of defaults, basic properties of default inference, inheritance and irrelevance, expressive power, and the lottery paradox.

\subsection{Semantics of defaults}

It is possible to discuss some properties of default reasoning systems in an extremely abstract fashion (see Section 3.2), but for other properties we need to make some assumptions about the type of system being considered. In particular, we consider systems that incorporate some notion of a default rule, which we now explain. In general, a default rule is an expression that has the form $A(x) \rightarrow B(x)$, whose intuitive interpretation is that if $A$ holds for some individual $x$ then typically (normally, usually, probably, etc.) $B$ holds for that individual. ${ }^{3}$ While the syntax actually used differs significantly from case to case, most default reasoning systems have some construct of this type. For instance, in Reiter's default logic [Rei80] we would write

$$
\frac{A(x): B(x)}{B(x)}
$$

while in a circumscriptive framework [McC80], we might use

$$
\forall x(A(x) \wedge \neg A b(x) \Rightarrow B(x))
$$

\footnotetext{
${ }^{3}$ We use $\rightarrow$ for a default implication, reserving $\Rightarrow$ for standard material implication.
} 
while circumscribing $A b(x)$. Theories based on first-order conditional logic [Del88] often do use the syntax $A(x) \rightarrow B(x)$. As we said in the introduction, in the random worlds framework this default is captured using the statistical assertion $\|B(x) \mid A(x)\|_{x} \approx 1$.

While most systems of default inference have a notion of a default rule, not all of them address the issue of what the rule means. In particular, while all systems describe how a default rule should be used, some do not ascribe semantics (or ascribe only unintuitive semantics) to such rules. Without a good, intuitive semantics for defaults it becomes very difficult to judge the reasonableness of a collection of default statements. For example, as we mentioned above, one standard reading of $\varphi \rightarrow \psi$ is " $\varphi$ 's are typically $\psi$ 's". Under this reading, the pair of defaults $A \rightarrow B$ and $A \rightarrow \neg B$ should be inconsistent. In approaches such as Reiter's default logic, $A \rightarrow B$ and $A \rightarrow \neg B$ can be simultaneously adopted; they are not "contradictory" because there is no relevant notion of contradiction.

In contrast, our approach does give semantics to defaults. In fact, we use a single logic and semantics that covers first-order information, default information, and statistical information. Such an approach enables us, among other things, to verify the consistency of a collection of defaults and to see whether a default follows logically from a collection of defaults. Of other existing theories, those based on conditional or modal logic come closest to achieving this (see [Bou91] for further discussion of this point).

\subsection{Properties of default inference}

As we said, default reasoning systems have typically been measured by testing them on a number of important examples. Recently, a few tools have been developed that improve upon this approach. Gabbay [Gab84] (and later Makinson [Mak89] and Kraus, Lehmann, and Magidor [KLM90]) introduced the idea of investigating the input/output relation of a default reasoning system, with respect to certain general properties that such an inference relation might possess. Makinson [Mak94] gives a detailed survey of this work.

The idea is simple. Fix a theory of default reasoning and let $K B$ be some knowledge base appropriate to this theory. Suppose $\varphi$ is a default conclusion reached from $K B$ according to the particular default approach being considered. In this case, we write $K B \sim \varphi$. The relation $\sim$ clearly depends on the default theory being considered. It is necessary to assume in this context that $K B$ and $\varphi$ are both expressed in the same logical language, and that the language has a notion of valid implication. Thus, for example, if we are considering default logic or $\epsilon$-semantics, we must assume that the defaults are fixed (and incorporated into the notion of $\sim$ ) and that both $K B$ and $\varphi$ are first-order or propositional formulas. Similarly, in the case of circumscription, the circumscriptive policy must also be fixed and incorporated into $\sim$. (See also the discussion at the beginning of Section 3.3.)

With this machinery we can state a few desirable properties of default theories in a way that is independent of the (very diverse) details of such theories. There are five properties of $\sim$ that have been viewed as being particularly desirable [KLM90]:

- Right Weakening. If $\varphi \Rightarrow \psi$ is logically valid and $K B \sim \varphi$, then $K B \sim \psi$.

- Reflexivity. $K B \sim K B$. 
- Left Logical Equivalence. If $K B \Leftrightarrow K B^{\prime}$ is logically valid, then $K B \sim \varphi$ if and only if $K B^{\prime} \sim \varphi$.

- Cut. If $K B \sim \theta$ and $K B \wedge \theta \sim \varphi$ then $K B \sim \varphi$.

- Cautious Monotonicity. If $K B \sim \theta$ and $K B \sim \varphi$ then $K B \wedge \theta \sim \varphi$.

While it is beyond the scope of this paper to defend these criteria (see [KLM90]), we do want to stress Cut and Cautious Monotonicity, since they will be useful in our later results. They tell us that we can safely add to $K B$ any conclusion $\theta$ that we can derive from $K B$, where "safely" is interpreted to mean that the set of conclusions derivable (via $\sim$ ) from $K B \wedge \theta$ is precisely the same as that derivable from $K B$ alone.

As shown in [KLM90], numerous other conditions can be derived from these properties. For example, we can prove:

- And. If $K B \sim \varphi$ and $K B \sim \psi$ then $K B \sim \varphi \wedge \psi$.

Other plausible properties, however, do not follow from these basic five. For example, the following property captures reasoning by cases:

- Or. If $K B \sim \varphi$ and $K B^{\prime} \sim \varphi$, then $K B \vee K B^{\prime} \sim \varphi$.

Perhaps the most interesting property that does not follow from the basic five properties is what has been called Rational Monotonicity [KLM90]. Note that the property of (full) monotonicity, which we do not want, says that $K B \sim \varphi$ implies $K B \wedge \theta \sim \varphi$, no matter what $\theta$ is. It has been argued that default reasoning should satisfy the same property in those cases where $\theta$ is "irrelevant" to the connection between $K B$ and $\varphi$. While it is difficult to characterize "irrelevance", one situation where we may believe that $\theta$ should not affect the conclusions we can derive from $K B$ is if $\theta$ is not implausible given $K B$, i.e., if it is not the case that $K B \sim \neg \theta$ (see Section 3.3 for an example). The following property asserts that monotonicity holds when adding such a formula $\theta$ to our knowledge base:

- Rational Monotonicity. If $K B \sim \varphi$ and it is not the case that $K B \sim \neg \theta$, then $K B \wedge \theta \sim \varphi$.

Rational Monotonicity is a fairly strong property, and is certainly not universally agreed upon (see [Mak94] for a discussion, and some weakened versions). However, several people, notably Lehmann and Magidor [LM92], have argued strongly for the desirability of this principle. One advantage of Rational Monotonicity is that it covers some fairly noncontroversial patterns of reasoning involving property inheritance. We explore this further in the next section. As is demonstrated in Section 5.1, our approach satisfies a slightly weakened version of Rational Monotonicity.

The set of properties we have discussed provides a simple, but useful, system for classifying default theories. There are certainly applications in which some of the properties are inappropriate; Reiter's default logic is still popular even though it does not satisfy Cautious Monotonicity, Or, or Rational Monotonicity [Mak94]. (We briefly discuss one of the consequent disadvantages of default logic in the next section.) Nevertheless, many people would argue that the five core properties given above constitute a reasonable, if incomplete, set of desiderata for mainstream default theories. 


\subsection{Specificity and inheritance}

As we have pointed out, systems of default reasoning have particular mechanisms for expressing default rules. A collection of such rules (perhaps in conjunction with other information) forms a default theory (or default knowledge base). For example, a particular default theory $K B_{\text {def }}$ might contain the default " $A$ 's are typically $B$ 's"; we denote this by writing $(A(x) \rightarrow B(x)) \in$ $K B_{\text {def }}$. A default theory $K B_{\text {def }}$ is used by a default reasoning system in order to reason from various premises to default conclusions. For example, a theory $K B_{\text {def }}$ containing the above default might infer $B(c)$ from $A(c)$. Let $\sim_{\text {def }}$ indicate the input/output relationship generated by a particular default reasoning system that uses $K B_{d e f}$. Thus, $A(c) \sim_{\text {def }} B(c)$ indicates that this default reasoning system is able to conclude $B(c)$ from the premise $A(c)$ using the default theory $K B_{\text {def. }}$. In this section we examine some additional properties we might like $\sim_{\text {def }}$ to satisfy.

Clearly, the presence of a default rule in a theory does not necessarily mean that the associated default reasoning system will (or should) apply that rule to any particular individual. Nevertheless, unless something special is known about that individual, the following seems to be an obvious requirement for any default reasoning system:

- Direct Inference for Defaults. If $(A(x) \rightarrow B(x)) \in K B_{\text {def }}$ and $K B_{\text {def }}$ contains no assertions mentioning $c$, then $A(c) \sim_{\text {def }} B(c)$.

This requirement has been previously discussed by Poole [Poo91], who called it the property of Conditioning. We have chosen a different name that relates the property more directly to earlier notions arising in work on direct inference.

We view Direct Inference for Defaults as stating a (very weak) condition for how a default theory should behave on some of the simpler problems involving hierarchies of classes and default properties. Consider the following standard example, in which our default knowledge base $K B_{f l y}$ is

$$
\begin{aligned}
& \operatorname{Bird}(x) \rightarrow F l y(x) \\
& \operatorname{Penguin}(x) \rightarrow \neg F l y(x), \\
& \forall x(\operatorname{Penguin}(x) \Rightarrow \operatorname{Bird}(x)) .
\end{aligned}
$$

Should Tweety the penguin inherit the property of flying from the class of birds, or the property of not flying from the class of penguins? For any system satisfying Direct Inference for Defaults we must have Penguin(Tweety) $\sim_{f l y} \neg F l y($ Tweety). So long as the system treats universals in a reasonable manner, this will be equivalent to Penguin(Tweety) $\wedge$ $\operatorname{Bird}($ Tweety $) \sim_{f l y} \neg F l y($ Tweety). Thus we see that if a system satisfies Direct Inference for Defaults, then it automatically satisfies a form of specificity - the preference for more specific defaults. Specificity in default reasoning is, of course, directly related to the preference for more specific subsets that we saw in the context of reference-class reasoning. Specificity is one of the least controversial desiderata in default reasoning.

In approaches such as default reasoning or circumscription, the most obvious encoding of these defaults satisfies neither Direct Inference for Defaults nor specificity. However, default logic and circumscription are certainly powerful enough for us to be able to arrange specificity if we wish. For example, in default logic, this can be done by means of non-normal defaults 
[RC81]. There is a cost to doing this, however: adding a default rule can require that all older default rules be reexamined, and possibly changed, to enforce the desired precedences.

Direct Inference for Defaults is a weak principle, since in most interesting cases there is no default that fits the case at hand perfectly. Suppose we learn that Tweety is a yellow penguin. Should we still conclude that Tweety does not fly? That is, should we conclude Penguin $($ Tweety $) \wedge$ Yellow (Tweety) $\sim_{f l y} \neg F l y($ Tweety)? Most people would say we should, because we have been given no reason to suspect that yellowness is relevant to flight. In other words, in the absence of more specific information about yellow penguins we should use the most specific superclass for which we do have knowledge, namely penguins. The inheritance property, i.e., the ability to inherit defaults from superclasses, is a second criterion for successful default reasoning, and is not provided by Direct Inference for Defaults.

In some sense, we can view Rational Monotonicity as providing a partial solution to this problem [LM92]. If a nonmonotonic reasoning system satisfies Rational Monotonicity in addition to Direct Inference for Defaults then it does achieve inheritance in a large number of examples. For instance, we have already observed that Direct Inference for Defaults gives Penguin (Tweety) $\sim_{f l y} \neg F l y\left(\right.$ Tweety), given $K B_{f l y}$. Since $K B_{f l y}$ gives us no reason to believe that yellow penguins are unusual, any reasonable default reasoning system would have Penguin(Tweety) $\downarrow_{\text {fly }} \neg$ Yellow(Tweety). From these two statements, Rational Monotonicity allows us to conclude Penguin $\left(\right.$ Tweety) $\wedge$ Yellow (Tweety) $\sim_{f l y} \neg F l y$ (Tweety), as desired.

However, Rational Monotonicity is still insufficient for inheritance reasoning in general. Suppose we add the default $\operatorname{Bird}(x) \rightarrow W a r m-b l o o d e d(x)$ to $K B_{f l y}$. We would surely expect Tweety to be warm-blooded. However, Rational Monotonicity cannot be applied here. To see why, observe that Bird (Tweety) $\sim_{f l y}$ Warm-blooded(Tweety), while we want to conclude that Bird (Tweety) $\wedge$ Penguin $($ Tweety $) \sim_{f l y}$ Warm-blooded (Tweety). ${ }^{4}$ We could use Rational Monotonicity to go from the first statement to the second, if we could show that $\operatorname{Bird}\left(\right.$ Tweety) $\not_{f l y} \neg$ Penguin(Tweety). However, most default reasoning systems do not support this statement. In fact, since penguins are exceptional birds that do not fly, it is not unreasonable to conclude the contrary, i.e., that Bird (Tweety) $\sim_{f l y} \neg$ Penguin $($ Tweety). Thus, Rational Monotonicity cannot be used to conclude that Tweety the penguin is warm-blooded.

It seems undesirable that if a subclass is exceptional in any one respect, then inheritance of all other properties is blocked. However, it can be argued that this blocking of inheritance to exceptional subclasses is reasonable. Since penguins are known to be exceptional birds perhaps we should be cautious and not allow them to inherit any of the normal properties of birds. But even if we accept this argument, there are many examples which demonstrate that the complete blocking of inheritance to exceptional subclasses yields an inappropriately weak theory of default reasoning. For example, suppose we add to $K B_{f l y}$ the default Yellow $(x) \rightarrow$ Easy-to-see $(x)$. This differs from standard exceptional-subclass inheritance in that yellow penguins are not known to be exceptional members of the class of yellow things. That is, while penguins are known to be somewhat unusual birds (and so perhaps the normal properties of birds should not be inherited), there is no reason to suppose that yellow penguins are different from other yellow objects. Nevertheless, Rational Monotonicity does not suffice even in this less controversial case. Indeed, there are well-known systems that satisfy Rational Monotonicity but cannot conclude

\footnotetext{
${ }^{4}$ In any system that treats universals reasonably, this is clearly equivalent to the assertion we are really interested in: Penguin (Tweety) $\sim_{f l y}$ Warm-blooded(Tweety).
} 
that Tweety, the yellow penguin, is easy to see [LM92, Pea90]. This problem has been called the drowning problem [Ash93, $\mathrm{BCD}^{+}$93].

Theories of default reasoning have had considerable difficulty in capturing an ability to inherit from superclasses that can deal properly with all of these different cases. In particular, the problem of inheritance to exceptional subclasses has been the most difficult. While some recent propositional theories have been more successful at dealing with exceptional subclass inheritance [GMP90, Gef92, GP92], they encounter other difficulties, which we discuss in the next section.

\subsection{Expressivity}

In the effort to discover basic techniques and principles for default reasoning, people have often looked at weak languages based on propositional logic. For instance, $\epsilon$-semantics and variants [GP90, GMP90], modal approaches such as autoepistemic logic [Moo85], and conditional logics [Bou91], are usually considered in a propositional framework. Others, such as Reiter's default logic and Delgrande's conditional logic [Del88], use a first-order language, but with a syntax that tends to decouple the issues of first-order reasoning and default reasoning; we discuss this below. Of the better-known systems, circumscription seems to have the ability, at least in principle, of making the richest use of first-order logic.

It seems uncontroversial that, ultimately, a system of default reasoning should be built around a powerful language. Sophisticated knowledge representation systems almost invariably use languages with the expressive power of some large fragment of first-order logic, if not much more. It is hard or impractical to encode the knowledge we have about almost any interesting domain without the expressive power provided by non-unary predicates and firstorder quantifiers. We would also like to reason logically as well as by default within the same system, and to allow perhaps even richer languages.

It has not been easy to integrate first-order logic and defaults completely. In fact, one of the major contributions of our approach is its ability to express both types of information in a single language. One difficulty for other approaches concerns "open" defaults, that are intended to apply to all individuals. For instance, suppose we wish to make a general statement that birds typically fly, and be able to use this when reasoning about different birds. Let us examine how some existing systems do this.

In propositional approaches, the usual strategy is to claim that there are different types of knowledge (see, for example, [GP92] and the references therein). General defaults, such as Bird $\rightarrow$ Fly, are in one class. When we reason about an individual, such as Tweety, its properties are described by knowledge in a different class, the context. For Tweety, the context might be Bird $\wedge$ Yellow. In a sense, the symbol Bird stands for a general property when used in a default and talks about Tweety (say) when it appears in the context. First-order approaches have more expressive power in this regard. For example, Reiter's default logic uses defaults with free variables, e.g., Bird $(x) \rightarrow F l y(x)$. That Tweety is a bird can then be written Bird(Tweety), which seems much more natural. The default itself is treated essentially as a schema, implying all substitution instances (such as Bird (Tweety) $\rightarrow$ Fly(Tweety)).

One example shows the difficulties with both of these approaches. Suppose we know that:

Elephants typically like zookeepers. 
Fred is a zookeeper, but elephants typically do not like Fred.

Clyde is an elephant.

Eric is a zookeeper.

Using this information we can apply specificity to determine reasonable answers to such questions as "Does Clyde like Fred?" (No) or "Does Clyde like Eric" (Yes). But the propositional strategy of classifying knowledge seems to fail here. Is "Elephants typically do not like Fred" a general default, or an item of contextual knowledge? Since it talks about elephants in general and also about one particular zookeeper, it does not fit either category well. In a rich first-order language, there is no clear-cut distinction between specific facts and general knowledge (nor do we believe there should be one).

Next, consider the first-order substitutional approach. It is easy to see that this does not work at all. One substitution instance of

$$
\text { Elephant }(x) \wedge \text { Zookeeper }(y) \rightarrow \operatorname{Likes}(x, y)
$$

is

$$
\text { Elephant }(x) \wedge \text { Zookeeper }(\text { Fred }) \rightarrow \operatorname{Likes}(x, \text { Fred }),
$$

which will contradict the second default. Of course, we could explicitly exclude Fred:

$$
\text { Elephant }(x) \wedge \text { Zookeeper }(y) \wedge y \neq \text { Fred } \rightarrow \text { Likes }(x, y) \text {. }
$$

However, explicit exclusion is similar to the process of explicitly disabling less specific defaults, mentioned in the previous section. Both destroy the modularity of the knowledge base, i.e., the form of a default becomes dependent on what other defaults are in the knowledge base. Hence, these techniques are highly impractical for large knowledge bases.

The zookeeper example is similar to an example given by Lehmann and Magidor [LM90]. However, the solution they suggest to this problem does not provide an explicit interpretation for open defaults. Rather, the "meaning" of an open default is implicitly determined by a set of rules provided for manipulating such defaults. These rules can cope with the zookeeper example, but the key step in the application of these rules is the use of Rational Monotonicity. More precisely, Lehmann and Magidor's argument applies to systems which, given the premise Elephant $(x) \wedge$ Zookeeper $(y)$, can infer by default that Likes $(x, y)$ (i.e., Elephant $(x) \wedge$ Zookeeper $(y) \sim \operatorname{Likes}(x, y))$, and yet cannot infer either $x \neq C l y d e$ or $y \neq$ Eric. The latter certainly seem reasonable since we know nothing whatsoever about Clyde or Eric. Now, however, we can apply Rational Monotonicity twice, which effectively allows us to assume (i.e., add to the premises) that $x=C l y d e \wedge y=$ Eric, while still concluding Likes $(x, y)$. Finally, Reflexivity, Right Weakening, and Left Logical Equivalence can be used to justify substituting for $x$ and $y$; we obtain Elephant $($ Clyde $) \wedge$ Zookeeper $($ Eric $) \sim$ Likes (Clyde,Eric), as desired. The key point is that this argument will typically fail for Fred, because we do have reason to believe that Fred is unusual (and so, in many systems, we could conclude by default that $y \neq$ Fred). Thus, as we would hope, we cannot conclude that Likes(Clyde, Fred), and in fact it is easy to argue analogously that we conclude $\neg$ Likes (Clyde, Fred) using the second default. But while Rational Monotonicity helps in this example, we have, in Section 3.3, already seen its main failing: it is easily blocked by "irrelevant" exceptionality. For example, if Eric is known to be exceptional 
in some way (even one unrelated to zookeeping), then Lehmann and Magidor's approach will not be able conclude that he is liked by Clyde. This is surely undesirable.

Thus, it seems to be very hard to interpret generic (open) defaults properly. This is perhaps the best-known issue regarding the expressive power of various approaches to default logic. There are, of course, others; we close by mentioning one.

Morreau [Mor93] has discussed the usefulness of being able to refer to "the class of individuals satisfying a certain default". For example, the assertion:

Typically, people who normally go to bed late normally rise late.

refers to "the class of people who normally go to bed late". The structure of this assertion is essentially:

$$
(\operatorname{Day}(y) \rightarrow \text { To-bed-late }(x, y)) \rightarrow\left(\operatorname{Day}\left(y^{\prime}\right) \rightarrow \text { Rises-late }\left(x, y^{\prime}\right)\right) .
$$

This is a default whose precondition and conclusion are descriptions of people whose behaviors are themselves defined using defaults. Such defaults appear to pose problems for most existing default theories. Reiter's default logic cannot express such defaults. And while some theories of conditional logic (for example, those of [Del88, Bou91]) can express this example, they are as yet incapable of generating reasonable inferences from nested defaults of this type. Circumscription, on the other hand, could perhaps be configured to cope with this example, but precisely how this could be accomplished is not obvious to us. We also note that the example has many variants. For instance, there is clearly a difference between the above default and the one "Typically, people who go to bed late rise late (i.e., the next morning)"; formally, the latter statement could be written:

$$
(\operatorname{Day}(y) \wedge \text { To-bed-late }(x, y)) \rightarrow \text { Rises-late }(x, N e x t \text {-day }(y))),
$$

There are also other variations. We would like to express and reason correctly with them all. The real issue here is that we need to define various properties of individuals, and while many of these properties can be expressed in first-order logic, others need to refer to defaults explicitly. This argues, yet again, that it is a mistake to have a different language for defaults than the one used for other knowledge.

\subsection{The lottery paradox}

The lottery paradox ([Kyb61]) addresses the issue of how different default conclusions interact. It provides a challenging test of the intuitions and semantics of any default reasoning system. There are a number of issues raised by this paradox; we consider three here.

First, imagine that a large number $N$ of people buy tickets to a lottery in which there is only one winner. For a particular person $c$, it seems sensible to conclude by default that $c$ does not win the lottery. But we can argue this way for every individual, which seems to contradict the fact that someone definitely will win. Of course some theories, such as those based on propositional languages, do not have enough expressive power to even state this version of the problem. Among theories that can state it, there would seem to be several options. Clearly, one 
solution is to deny that default conclusions are closed under arbitrary conjunction, i.e., to give up on the And Rule. But aside from explicitly probabilistic theories, we are not aware of work taking this approach (although the existence of multiple extensions in theories such as Reiter's is certainly related). Without logical closure, there is a danger of being too dependent on merely syntactic features of a problem. Another solution is to prevent a theory from reasoning about all $N$ individuals at once [EKP91]. Finally, one can simply deny that $\neg$ Winner $(c)$ follows by default. Circumscription, for instance, does this: The standard representation of the problem would result in multiple extensions, such that for each individual $c$, there is one extension where $c$ is the winner. While this seems reasonable, circumscription only allows us to conclude things that hold in all extensions; thus, we would not be able to conclude $\neg$ Winner $(c)$. The problem with these "solutions" is that the lottery problem seems to be an extremely reasonable application of default reasoning: if you buy a lottery ticket you should continue your life under the assumption that you will not win.

Second, a closely related issue is raised by Lifschitz's list of benchmark problems [Lif89]. Suppose we have a default, for instance Ticket $(x) \rightarrow \neg \operatorname{Winner}(x)$, and no other knowledge. Should $\forall x$ (Ticket $(x) \Rightarrow \neg$ Winner $(x))$ be a default conclusion? Likewise, if we know Winner $(c)$ but consider it possible that the lottery has more than one winner, should we nevertheless conclude that $\forall x(($ Ticket $(x) \wedge x \neq c) \Rightarrow \neg$ Winner $(x))$ ? In circumscription, although not in many other theories, we get both universal conclusions (as Lifschitz argues for). The desire for these universal conclusions is certainly controversial; in fact it seems that we often expect default rules to have some exceptions. However, as Lifschitz observes, there is a technical difficulty in following this latter intuition: How can we conclude from the default Ticket $(x) \rightarrow \neg$ Winner $(x)$ that, by default, each individual $c$ is not a winner, and yet not also reach the universal conclusion that, by default, no one wins? The concern is that, in many systems, the latter conclusion will be logically entailed whether we wish it or not. Because of its treatment of open defaults, Reiter's default logic does not suffer from this difficulty. As we shall see, neither does the random-worlds approach.

Finally, Poole [Poo91] has considered a variant of the lottery paradox that avoids entirely the issue of named individuals. In his version, there is a formula describing the types of birds we are likely to encounter, such as:

$$
\forall x(\operatorname{Bird}(x) \Leftrightarrow(\operatorname{Emu}(x) \vee \operatorname{Penguin}(x) \vee \ldots \vee \operatorname{Canary}(x)))
$$

We then add to the knowledge base defaults such as birds typically fly, but penguins typically do not fly, and we similarly assert that every other species of bird is exceptional in some way. Now suppose all we know is that Bird(Tweety). Can we conclude that Tweety flies? If we conclude that he can, then a similar argument would also allow us to conclude that he is a typical bird in all other respects. But this would contradict the fact he must be exceptional in some respect. If we do not conclude that Tweety flies, then the default "Birds typically fly" has been effectively ignored. Poole uses such examples to give an exhaustive analysis of how various systems might react to the Lottery Paradox. He shows that in any theory, some desideratum, such as closure under conjunction or "conditioning" (Direct inference for defaults), must be sacrificed. Perhaps the most interesting "way out" he discusses is the possibility of declaring that certain combinations of defaults are inadmissible or inconsistent. Is it really reasonable to say that the class of birds is the union of subclasses all of which are exceptional? In many 
theories, such as Reiter's default logic, there is nothing to prevent one from asserting this. But in a theory which gives reasonable semantics to defaults, we may be able to determine and justify the incompatibility of certain sets of defaults. This, indeed, is how our approach avoids Poole's version of the lottery paradox.

\subsection{Discussion}

In this section, we have presented a limited list of desiderata that seem appropriate for a default reasoning system, and have discussed some key problems and issues that must be resolved by such a system. While our list may be limited, it is interesting to point out that there does not seem to be a single default reasoning system that fulfills all these desiderata in a satisfactory way. Although we can (and do) show that random worlds does, in fact, achieve all the requirements on this list, we would like to validate random worlds in a more comprehensive fashion. Unfortunately, to the best of our knowledge, there is (as yet) no general framework for evaluating default reasoning systems. In particular, evaluation still tends to be on the level of "Does this theory solve these particular examples correctly?" (see, for example, the list of benchmark problems in [Lif89]). While such examples are often important in identifying interesting aspects of the problem and defining our intuitions in these cases, they are clearly not a substitute for a comprehensive framework. Had there been such a framework, perhaps the drowning problem from Section 3.3 would not have remained undiscovered for so long. While we do not attempt to provide such a general framework in this paper, in Section 5 we prove a number of general theorems concerning the random-worlds approach. These theorems provide a precise formulation of properties such as Direct Inference for Defaults, and show that they hold for random worlds. Other properties such as specificity and exceptional subclass inheritance follow immediately from these theorems. Thus, our proof that the random-worlds approach deals well with the paradigm examples in default reasoning follows from a general theorem, rather than by a case-by-case analysis.

\section{The formalism}

\subsection{The language}

We are interested in a formal logical language that allows us to express both statistical information and first-order information. We therefore define a statistical language $\mathcal{L} \approx$, which is a variant of a language designed by Bacchus [Bac90]. For the remainder of the paper, let $\Phi$ be a finite first-order vocabulary, consisting of predicate, function, and constant symbols, and let $\mathcal{X}$ be a set of variables.

Our statistical language augments standard first-order logic with a form of statistical quantifier. For a formula $\psi(x)$, the term $\|\psi(x)\|_{x}$ is a proportion expression. It will be interpreted as a rational number between 0 and 1 , that represents the proportion of domain elements satisfying $\psi(x)$. We actually allow an arbitrary set of variables in the subscript and in the formula $\psi$. Thus, for example, $\|$ Child $(x, y) \|_{x}$ describes, for a fixed $y$, the proportion of domain elements that are children of $y$; $\|$ Child $(x, y) \|_{y}$ describes, for a fixed $x$, the proportion of domain elements whose child is $x$; and $\|\operatorname{Child}(x, y)\|_{x, y}$ describes the proportion of pairs of domain elements that are in the child relation. 
We also allow proportion expressions of the form $\|\psi(x) \mid \theta(x)\|_{x}$, which we call conditional proportion expressions. Such an expression is intended to denote the proportion of domain elements satisfying $\psi$ from among those elements satisfying $\theta$. Finally, any rational number is also considered to be a proportion expression, and the set of proportion expressions is closed under addition and multiplication.

One important difference between our syntax and that of [Bac90] is the use of approximate equality to compare proportion expressions. As we argued in the introduction, exact comparisons are sometimes inappropriate. Consider a statement such as " $80 \%$ of patients with jaundice have hepatitis". If this statement appears in a knowledge base, it is almost certainly there as a summary of a large pool of data. It is clear that we do not mean that exactly $80 \%$ of all patients with jaundice have hepatitis. Among other things, this would imply that the number of jaundiced patients is a multiple of five, which is surely not an intended implication. We therefore use the approach described in [GHK94, KH92], and compare proportion expressions using (instead of $=$ and $\leq$ ) one of an infinite family of connectives $\approx_{i}$ and $\preceq_{i}$, for $i=1,2,3 \ldots$ (" $i$-approximately equal" or " $i$-approximately less than or equal"). ${ }^{5}$ For example, we can express the statement " $80 \%$ of jaundiced patients have hepatitis" by the proportion formula $\|\operatorname{Hep}(x) \mid \operatorname{Jaun}(x)\|_{x} \approx_{1}$ 0.8. The intuition behind the semantics of approximate equality is that each comparison should be interpreted using some small tolerance factor to account for measurement error, sample variations, and so on. The appropriate tolerance will differ for various pieces of information, so our logic allows different subscripts on the "approximately equals" connectives. A formula such as $\left\|F l y(x)\left|\operatorname{Bird}(x)\left\|_{x} \approx_{1} 1 \wedge\right\| F l y(x)\right| \operatorname{Bat}(x)\right\|_{x} \approx_{2} 1$ says that both $\|F l y(x) \mid \operatorname{Bird}(x)\|_{x}$ and $\|F l y(x) \mid \operatorname{Bat}(x)\|_{x}$ are approximately 1, but the notion of "approximately" may be different in each case.

We can now give a recursive definition of the language $\mathcal{L} \approx$.

Definition 4.1: The set of terms in $\mathcal{L}^{\approx}$ is the least set containing $\mathcal{X}$ and the constant symbols in $\Phi$ that is closed under function application (so that if $f$ is a function symbol in $\Phi$ of arity $r$, and $t_{1}, \ldots, t_{r}$ are terms, then so is $\left.f\left(t_{1}, \ldots, t_{r}\right)\right)$.

The set of proportion expressions is the least set that

(a) contains the rational numbers,

(b) contains proportion terms of the form $\|\psi\|_{X}$ and $\|\psi \mid \theta\|_{X}$, for formulas $\psi, \theta \in \mathcal{L}^{\approx}$ and a finite set of variables $X \subseteq \mathcal{X}$, and

(c) is closed under addition and multiplication.

The set of formulas in $\mathcal{L}^{\approx}$ is the least set that

(a) contains atomic formulas of the form $R\left(t_{1}, \ldots, t_{r}\right)$, where $R$ is a predicate symbol in $\Phi \cup\{=\}$ of arity $r$ and $t_{1}, \ldots, t_{r}$ are terms,

(b) contains proportion formulas of the form $\zeta \approx_{i} \zeta^{\prime}$ and $\zeta \preceq_{i} \zeta^{\prime}$, where $\zeta$ and $\zeta^{\prime}$ are proportion expressions and $i$ is a natural number, and

\footnotetext{
${ }^{5}$ In [BGHK92] the use of approximate equality was suppressed in order to highlight other issues.
} 
(c) is closed under conjunction, negation, and first-order quantification.

Notice that this definition allows arbitrary nesting of quantifiers and proportion expressions. In Section 4.3 we demonstrate the expressive power of the language. As observed in [Bac90], the appearance of a variable $x$ in the subscript of a proportion expression binds the variable $x$ in the expression; indeed, we can view $\|\cdot\|_{X}$ as a new type of quantification.

We now need to define the semantics of the logic. As we shall see below, most of the definitions are fairly straightforward. The two features that cause problems are approximate comparisons and conditional proportion expressions. We interpret the approximate connective $\zeta \approx_{i} \zeta^{\prime}$ to mean that $\zeta$ is very close to $\zeta^{\prime}$. More precisely, it is within some very small, but unknown, tolerance factor. We formalize this using a tolerance vector $\left.\vec{\tau}=\left\langle\tau_{1}, \tau_{2}, \ldots\right\rangle, \tau_{i}\right\rangle 0$. Intuitively $\zeta \approx_{i} \zeta^{\prime}$ if the values of $\zeta$ and $\zeta^{\prime}$ are within $\tau_{i}$ of each other. (Note that, although the use of tolerance vectors leads to well-defined formal semantics, one might object that in practice we generally will not know appropriate tolerance values. We defer our response to this objection to the next section.)

A difficulty arises when interpreting conditional proportion expressions because we need to deal with the problem of conditioning on an event of measure 0 . That is, we need to define semantics for $\|\psi \mid \theta\|_{X}$ even when there are no assignments to the variables in $X$ that would satisfy $\theta$. When standard equality is used rather than approximate equality, this problem is easily overcome. Following [Hal90], we can eliminate conditional proportion expressions altogether by viewing a statement such as $\|\psi \mid \theta\|_{X}=\alpha$ as an abbreviation for $\|\psi \wedge \theta\|_{X}=\alpha\|\theta\|_{X}$. This approach agrees with the standard interpretation of conditionals if $\|\theta\|_{X} \neq 0$. If $\|\theta\|_{X}=0$, it enforces the convention that formulas such as $\|\psi \mid \theta\|_{X}=\alpha$ or $\|\psi \mid \theta\|_{X} \leq \alpha$ are true for any $\alpha$. We used the same approach in [GHK94], where we allowed approximate equality. Unfortunately, as the following example shows, this interpretation of conditional proportions can interact in an undesirable way with the semantics of approximate comparisons. In particular, this approach does not preserve the standard semantics of conditional equality if $\|\theta\|_{X}$ is approximately 0 .

Example 4.2: Consider the knowledge base: ${ }^{6}$

$$
K B=\left(\|\operatorname{Penguin}(x)\|_{x} \approx_{1} 0\right) \wedge\left(\|F l y(x) \mid \operatorname{Penguin}(x)\|_{x} \approx_{2} 0\right) .
$$

We expect this to mean that the proportion of penguins is very small (arbitrarily close to 0 in large domains), but also that the proportion of fliers among penguins is also very small. However, if we attempt to interpret conditional proportions as discussed above, we obtain the knowledge base

$$
K B^{\prime}=\left(\|\operatorname{Penguin}(x)\|_{x} \approx_{1} 0\right) \wedge\left(\|F l y(x) \wedge \operatorname{Penguin}(x)\|_{x} \approx_{2} 0 \cdot\|\operatorname{Penguin}(x)\|_{x}\right),
$$

which is equivalent to

$$
\left(\|\operatorname{Penguin}(x)\|_{x} \approx_{1} 0\right) \wedge\left(\| \text { Fly }(x) \wedge \operatorname{Penguin}(x) \|_{x} \approx_{2} 0\right) .
$$

\footnotetext{
${ }^{6}$ We remark that, here and in our examples below, the actual choice of subscript for $\approx$ is unimportant. However, we use different subscripts for different approximate comparisons unless the tolerances for the different measurements are known to be the same.
} 
This last formula simply asserts that the proportion of penguins and the proportion of flying penguins are both small, but says nothing about the proportion of fliers among penguins. In fact, the world where all penguins fly is consistent with $K B^{\prime}$. Clearly, the process of multiplying out across an approximate connective does not preserve the intended interpretation of the formulas.

Because of this problem, we cannot treat conditional proportions as abbreviations and instead have added them as primitive expressions in the language. Of course, we now have to give them a semantics that avoids the problem illustrated by Example 4.2. We would like to maintain the conventions used when we had equality in the language. Namely, in worlds where $\|\theta(x)\|_{x} \neq 0$, we want $\|\varphi(x) \mid \theta(x)\|_{x}$ to denote the fraction of elements satisfying $\theta(x)$ that also satisfy $\varphi(x)$. In worlds where $\|\theta(x)\|_{x}=0$, we want all formulas of the form $\|\varphi(x) \mid \theta(x)\|_{x} \approx_{i} \alpha$ or $\|\varphi(x) \mid \theta(x)\|_{x} \preceq_{i} \alpha$ to be true. There are a number of ways of accomplishing this. The route we take is perhaps not the simplest, but it introduces machinery that will be helpful later.

We give semantics to the language $\mathcal{L}^{\approx}$ by providing a translation from formulas in $\mathcal{L}^{\approx}$ to formulas in a language $\mathcal{L}^{=}$whose semantics is more easily described. The language $\mathcal{L}^{=}$is essentially the language of [Hal90], that uses true equality rather than approximate equality. More precisely, the definition of $\mathcal{L}^{=}$is identical to the definition of $\mathcal{L}^{\approx}$ given in Definition 4.1, except that:

- we use $=$ and $\leq$ instead of $\approx_{i}$ and $\preceq_{i}$,

- we allow the set of proportion expressions to include arbitrary real numbers (not just rational numbers),

- we do not allow conditional proportion expressions,

- we assume that $\mathcal{L}^{=}$has a special family of variables $\varepsilon_{i}$, interpreted over the reals.

As we shall see, the variable $\varepsilon_{i}$ is used to interpret the approximate equality connectives $\approx_{i}$ and $\preceq_{i}$. We view an expression in $\mathcal{L}^{=}$that uses conditional proportion expressions as an abbreviation for the expression obtained by multiplying out.

The semantics for $\mathcal{L}^{=}$is quite straightforward, and follows the lines of [Hal90]. Recall that we give semantics to $\mathcal{L}^{=}$in terms of worlds, or finite first-order models. For any natural number $N$, let $\mathcal{W}_{N}(\Phi)$ consist of all worlds with domain $D=\{1, \ldots, N\}$ over the vocabulary $\Phi$.

Now, consider a world $W \in \mathcal{W}_{N}(\Phi)$, a valuation $V: \mathcal{X} \rightarrow\{1, \ldots, N\}$ for the variables in $\mathcal{X}$, and a tolerance vector $\vec{\tau}$. We simultaneously assign to each proportion expression $\zeta$ a real number $[\zeta]_{(W, V, \vec{\tau})}$ and to each formula $\xi$ a truth value with respect to $(W, V, \vec{\tau})$. Most of the clauses of the definition are completely standard, so we omit them here. In particular, variables are interpreted using $V$, each tolerance variable $\varepsilon_{i}$ is interpreted as denoting the tolerance $\tau_{i}$, the predicates and constants are interpreted using $W$, the Boolean connectives and the first-order quantifiers are defined in the standard fashion, and when interpreting proportion expressions, the real numbers, addition, multiplication, and $\leq$ are given their standard meaning. It remains to interpret proportion terms. Recall that we eliminate conditional proportion terms by multiplying out, so that we need to deal only with unconditional proportion terms. If $\zeta$ is 
the proportion expression $\|\psi\|_{x_{i_{1}}, \ldots, x_{i_{k}}}\left(\right.$ for $\left.i_{1}<i_{2}<\ldots<i_{k}\right)$, then

$$
[\zeta]_{(W, V, \vec{\tau})}=\frac{1}{|D|^{k}}\left|\left\{\left(d_{1}, \ldots, d_{k}\right) \in D^{k}:\left(W, V\left[x_{i_{1}} / d_{1}, \ldots, x_{i_{k}} / d_{k}\right], \vec{\tau}\right) \models \psi\right\}\right| .
$$

Thus, if $W \in \mathcal{W}_{N}(\Phi)$, the proportion expression $\|\psi\|_{x_{i_{1}}, \ldots, x_{i_{k}}}$ denotes the fraction of the $N^{k} k$ tuples of domain elements in $D$ that satisfy $\psi$ in the world $W$. For example, [\|Child $\left.(x, y) \|_{x}\right]_{(W, V, \vec{\tau})}$ is the fraction of domain elements $d$ that are children of $V(y)$.

We now show how a formula $\chi \in \mathcal{L}^{\approx}$ can be associated with a formula $\chi^{*} \in \mathcal{L}^{=}$. We proceed as follows:

- every proportion formula $\zeta{ }_{i} \zeta^{\prime}$ in $\chi$ is (recursively) replaced by $\zeta-\zeta^{\prime} \leq \varepsilon_{i}$,

- every proportion formula $\zeta \approx_{i} \zeta^{\prime}$ in $\chi$ is (recursively) replaced by the conjunction $\left(\zeta-\zeta^{\prime} \leq\right.$ $\left.\varepsilon_{i}\right) \wedge\left(\zeta^{\prime}-\zeta \leq \varepsilon_{i}\right)$

- finally, conditional proportion expressions are eliminated as in [Hal90]'s semantics, by multiplying out.

This translation allows us to embed $\mathcal{L}^{\approx}$ in $\mathcal{L}^{=}$. Thus, for the remainder of the paper, we regard $\mathcal{L} \approx$ as a sublanguage of $\mathcal{L}^{=}$. We can now easily define the semantics of formulas in $\mathcal{L}^{\approx}$ : For $\chi \in \mathcal{L}^{\approx}$, we say that $(W, V, \vec{\tau}) \models \chi$ iff $(W, V, \vec{\tau}) \models \chi^{*}$. It is sometimes useful to incorporate particular values for the tolerances into the formula $\chi^{*}$. Thus, let $\chi[\vec{\tau}]$ represent the formula that results from $\chi^{*}$ if each variable $\varepsilon_{i}$ is replaced by $\tau_{i}$, its value according to $\vec{\tau}^{7}$

Typically we are interested in closed sentences, that is, formulas with no free variables. In that case, it is not hard to show that the valuation plays no role. Thus, if $\chi$ is closed, we write $(W, \vec{\tau}) \models \chi$ rather than $(W, V, \vec{\tau}) \models \chi$.

\subsection{Degrees of belief}

As we explained in the introduction, we give semantics to degrees of belief by considering all worlds of size $N$ to be equally likely, conditioning on $K B$, and then checking the probability of $\varphi$ over the resulting probability distribution. In the previous section, we defined what it means for a sentence $\chi$ to be satisfied in a world of size $N$ using a tolerance vector $\vec{\tau}$. Given $N$ and $\vec{\tau}$, we define $\# \operatorname{worlds} s_{N}^{\vec{\tau}}(\chi)$ to be the number of worlds in $\mathcal{W}_{N}(\Phi)$ such that $(W, \vec{\tau}) \models \chi$. Since we are taking all worlds to be equally likely, the degree of belief in $\varphi$ given $K B$ with respect to $\mathcal{W}_{N}$ and $\vec{\tau}$ is

$$
\operatorname{Pr}_{N}^{\vec{\tau}}(\varphi \mid K B)=\frac{\# \text { worlds } s_{N}^{\vec{\tau}}(\varphi \wedge K B)}{\# \text { worlds } s_{N}^{\vec{\tau}}(K B)} .
$$

If $\# \operatorname{worlds}_{N}^{\vec{\tau}}(K B)=0$, this degree of belief is not well-defined. ${ }^{8}$

\footnotetext{
${ }^{7}$ Note that some of the tolerances $\tau_{i}$ may be irrational; it is for this reason that we allowed arbitrary real numbers in the proportion expressions of $\mathcal{L}^{=}$.

${ }^{8}$ Strictly speaking, we should write \# $\operatorname{world} s_{N}^{\Phi, \vec{\tau}}(\chi)$ rather than \#worlds $\vec{\tau}_{N}(\chi)$, since the number also depends on the choice of $\Phi$. Indeed, we do so in the one place where this dependence matters (Theorem 5.27). The degree of belief is, however unaffected by expansions of the vocabulary. That is, if $\Phi^{\prime} \supset \Phi$ then the degree of belief $\operatorname{Pr}_{N}^{\vec{\tau}}(\varphi \mid K B)$ is the same under the vocabulary $\Phi^{\prime}$ as it is under $\Phi$.
} 
Typically, we know neither $N$ nor $\vec{\tau}$ exactly. All we know is that $N$ is "large" and that $\vec{\tau}$ is "small". Thus, we would like to take our degree of belief in $\varphi$ given $K B$ to be $\lim _{\vec{\tau} \rightarrow \overrightarrow{0}} \lim _{N \rightarrow \infty} \operatorname{Pr}_{N}^{\vec{\tau}}(\varphi \mid K B)$. Notice that the order of the two limits over $\vec{\tau}$ and $N$ is important. If the $\operatorname{limit}_{\vec{\tau} \rightarrow \overrightarrow{0}}$ appeared last, then we would gain nothing by using approximate equality, since the result would be equivalent to treating approximate equality as exact equality.

This definition, however, is not sufficient; the limit may not exist. We observed above that $\operatorname{Pr}_{N}^{\vec{\tau}}(\varphi \mid K B)$ is not always well-defined. In particular, it may be the case that for certain values of $\vec{\tau}, \operatorname{Pr}_{N}^{\vec{\tau}}(\varphi \mid K B)$ is not well-defined for arbitrarily large $N$. In order to deal with this problem of well-definedness, we define $K B$ to be eventually consistent if for all sufficiently small $\vec{\tau}$ and sufficiently large $N$, \#worlds $s_{N}^{\vec{\tau}}(K B)>0$. Among other things, eventual consistency implies that the $K B$ is satisfiable in finite domains of arbitrarily large size. For example, a $K B$ stating that "there are exactly 7 domain elements" is not eventually consistent. ${ }^{9}$ For the remainder of the paper, we assume that all knowledge bases are eventually consistent.

Even if $K B$ is eventually consistent, the limit may not exist. For example, it may be the case that for some $i, \operatorname{Pr}_{N}^{\vec{\tau}}(\varphi \mid K B)$ oscillates between $\alpha+\tau_{i}$ and $\alpha-\tau_{i}$ as $N$ gets large. In this case, for any particular $\vec{\tau}$, the limit as $N$ grows will not exist. However, it seems as if the limit as $\vec{\tau}$ grows small "should", in this case, be $\alpha$, since the oscillations about $\alpha$ go to 0 . We avoid such problems by considering the lim sup and lim inf, rather than the limit. For any set $S \subset \mathbb{R}$, the infimum of $S$, inf $S$, is the greatest lower bound of $S$. The lim inf of a sequence is the limit of the infimums; that is,

$$
\liminf _{N \rightarrow \infty} a_{N}=\lim _{N \rightarrow \infty} \inf \left\{a_{i}: i>N\right\}
$$

The lim inf exists for any sequence bounded from below, even if the limit does not. The lim sup is defined analogously, where $\sup S$ denotes the least upper bound of $S$. If $\lim _{N \rightarrow \infty} a_{N}$ does exist, then $\lim _{N \rightarrow \infty} a_{N}=\liminf _{N \rightarrow \infty} a_{N}=\limsup _{N \rightarrow \infty} a_{N}$. Since, for any $\vec{\tau}$, the sequence $\operatorname{Pr}_{N}^{\vec{\tau}}(\varphi \mid K B)$ is always bounded from above and below, the lim sup and lim inf always exist. Thus, we do not have to worry about the problem of nonexistence for particular values of $\vec{\tau}$. We can now present the final form of our definition.

\section{Definition 4.3: If}

$$
\lim _{\vec{\tau} \rightarrow \overrightarrow{0}} \liminf _{N \rightarrow \infty} \operatorname{Pr}_{N}^{\vec{\tau}}(\varphi \mid K B) \text { and } \lim _{\vec{\tau} \rightarrow \overrightarrow{0}} \limsup _{N \rightarrow \infty} \operatorname{Pr}_{N}^{\vec{\tau}}(\varphi \mid K B)
$$

both exist and are equal, then the degree of belief in $\varphi$ given $K B$, written $\operatorname{Pr}_{\infty}(\varphi \mid K B)$, is defined as the common limit; otherwise $\operatorname{Pr}_{\infty}(\varphi \mid K B)$ does not exist.

We point out that, even using this definition, there are many cases where the degree of belief does not exist. However, as some of our examples show, in many situations the nonexistence of a degree of belief can be understood intuitively, and is sometimes related to the existence of multiple extensions of a default theory. (See Sections 4.3 and 5.3 and [GHK94].)

\footnotetext{
${ }^{9}$ Of course, in this case one probably would not want to consider $\lim N \rightarrow \infty$ anyway. If we are fortunate enough to know the domain size, and it is reasonably small, we can simply compute degrees of belief using the (known) fixed value of $N$.
} 
We remark that Shastri [Sha89] used a somewhat similar approach to defining degrees of belief. His language does not allow the direct expression of statistical information, but does allow us to talk about the number of domain individuals that satisfy a given predicate. He then gives a definition of degree of belief similar to ours. Since he has no notion of approximate equality in his language, and presumes a fixed domain size (an assumption we wish to avoid), he does not have to deal with limits as we do.

\subsection{Statistical interpretation for defaults}

As we mentioned in the introduction, there are many similarities between direct inference from statistical information and default reasoning. To capitalize on this observation, and to be able to use random worlds as a default reasoning system, we need to interpret defaults as statistical statements. However, finding the appropriate statistical interpretation is not straightforward. For example, as is well known, if we interpret "Birds typically fly" as "Most (i.e., more than $50 \%$ of) birds fly", then we get a default system that fails to satisfy some of the most basic desiderata, such as the And rule, discussed in Section 3.2. Using a higher fixed threshold in a straightforward way does not help. More successfully, Adams [Ada75], and later Geffner and Pearl [GP90], suggested an interpretation of defaults based on "almost all". In their framework, this is done using extreme probabilities - conditional probabilities that are arbitrarily close to 1: i.e., within $1-\epsilon$ for some $\epsilon$, and considering the limit as $\epsilon \rightarrow 0$. The basic system derived from this idea is called $\epsilon$-semantics. Later, stronger systems (that are able to make more inferences) based on the same probabilistic idea were introduced (see Pearl [Pea89] for a survey).

The intuition behind $\epsilon$-semantics and its extensions seems to be statistical. However, since the language used in these approaches is propositional, this intuition cannot be expressed directly. Indeed, these approaches typically make no distinction between the statistical nature of the default and the degree of belief nature of the default conclusion. We are able to capture this intuition more directly in our approach, since we can make this distinction explicitly. Recall that we interpret a statement such as "Birds typically fly" statistically, using the approximate statement $\|F l y(x) \mid \operatorname{Bird}(x)\|_{x} \approx_{i} 1$ for some $i$. (Thus, the use of an approximate connective to compare proportion expressions is not purely a technical convenience.) Clearly, we can view our statistical interpretation of defaults as a generalization of the extreme probabilities interpretation of defaults to the first-order case. The connection between our work and $\epsilon$-semantics extends beyond the issue of representation: there is a deeper sense in which we can view our approach as the generalization of one of the extensions of $\epsilon$-semantics, namely the maximumentropy approach of Goldszmidt, Morris, and Pearl [GMP90], to the first-order setting. This issue is discussed in more detail in Section 6, where it is shown that this maximum-entropy approach can be embedded in our framework.

Of course, the fact that our syntax is so rich allows us to express a great deal of information that simply cannot be expressed in any propositional approach. We observed earlier that a propositional approach that distinguishes between default knowledge and contextual knowledge has difficulty in dealing with the elephant-zookeeper example (see Section 3.4). This example is easily dealt with in our framework.

Example 4.4: The following knowledge base, $K B_{\text {likes }}$, is a formalization of the elephantzookeeper example. Recall, this problem concerns the defaults that (a) Elephants typically like 
zookeepers, but (b) Elephants typically do not like Fred. As discussed earlier, simply expressing this knowledge can be a challenge. In our framework this example can be expressed as follows:

$$
\begin{aligned}
& \| \text { Likes }(x, y) \mid \text { Elephant }(x) \wedge \text { Zookeeper }(y) \|_{x, y} \approx_{1} 1 \wedge \\
& \| \text { Likes }(x, \text { Fred }) \mid \text { Elephant }(x) \|_{x} \approx_{2} 0 \wedge \\
& \text { Zookeeper }(\text { Fred }) \wedge \text { Elephant }(\text { Clyde }) \wedge \text { Zookeeper }(\text { Eric }) .
\end{aligned}
$$

Furthermore, our interpretation of defaults allows us to deal well with interactions between first-order quantifiers and defaults.

Example 4.5: We may know that people who have at least one tall parent are typically tall. This default can be expressed in our language:

$$
\|\operatorname{Tall}(x) \mid \exists y(\operatorname{Child}(x, y) \wedge \operatorname{Tall}(y))\|_{x} \approx_{i} 1 .
$$

We can also define defaults over classes themselves defined using default rules (as discussed by Morreau [Mor93]).

Example 4.6: In Section 3.4, we discussed the problem of expressing the nested default "Typically, people who normally go to bed late normally rise late." To express this default we can simply use nested proportion statements: The individuals who normally rise late are those who rise late most days; they are the $x$ 's satisfying $\|$ Rises-late $(x, y) \mid \operatorname{Day}(y) \|_{y} \approx_{1} 1$. Similarly, the individuals who normally go to bed late are the $x$ 's satisfying $\|$ To-bed-late $\left(x, y^{\prime}\right) \mid \operatorname{Day}\left(y^{\prime}\right) \|_{y^{\prime}}$ $\approx_{2} 1$. Thus we can capture the default by saying most $x$ 's that go to bed late also rise late, as in the knowledge base $K B_{\text {late }}$ :

$$
\|\| \text { Rises-late }(x, y)\left|\operatorname{Day}(y)\left\|_{y} \approx_{1} 1 \mid\right\| \text { To-bed-late }\left(x, y^{\prime}\right)\right| \operatorname{Day}\left(y^{\prime}\right)\left\|_{y^{\prime}} \approx_{2} 1\right\|_{x} \approx_{3} 1 \text {. }
$$

On the other hand, the related default that "Typically, people who go to bed late rise late (i.e., the next morning)" can be expressed as:

$$
\| \text { Rises-late }(x, \operatorname{Next} \text {-day }(y)) \mid \operatorname{Day}(y) \wedge \text { To-bed-late }(x, y) \|_{x, y} \approx_{1} 1 \text {, }
$$

which is clearly different from the first default.

\section{Properties of random worlds}

We now show that the random-worlds method validates several desirable reasoning patterns, including essentially all of those discussed in Sections 2 and 3. It is worth noting that all of these reasoning patterns follow from the basic definition of the random worlds method given in Section 4.2; none of these patterns require any additional structure to be added to the method. We also note that all the results in this section hold for our language in its full generality: the formulas can contain arbitrary function and predicate symbols (including non-unary predicates), and have nested quantifiers and proportion statements. Finally, we note that the theorems we state are not the most general ones possible. It is quite easy to construct examples for which the conditions of the theorems do not hold, but random worlds still gives the intuitively plausible answer. We could find theorems that deal with additional cases, although it seems to be fairly difficult to find other results whose conditions are easy to state and check, and yet cover an interestingly large class of examples. We discuss this issue again in Section 7.4. 


\subsection{Random worlds and default reasoning}

In this subsection, we focus on formulas which are assigned degree of belief 1 . Given any knowledge base $K B$ (which can, in particular, include defaults using the statistical interpretation of Section 4.3), we say that $\varphi$ is a default conclusion from $K B$, and write $K B \sim_{r w} \varphi$, if $\operatorname{Pr}_{\infty}(\varphi \mid K B)=1$. As we now show, the relation $\sim_{r w}$ satisfies all the basic properties of default inference discussed in Section 3.2. We start by proving two somewhat more general results.

Proposition 5.1: If $\models K B \Leftrightarrow K B^{\prime}$, then $\operatorname{Pr}_{\infty}(\varphi \mid K B)=\operatorname{Pr}_{\infty}\left(\varphi \mid K B^{\prime}\right)$ for all formulas $\varphi .^{10}$

Proof: By assumption, precisely the same set of worlds satisfy $K B$ and $K B^{\prime}$. Therefore, for all $N$ and $\vec{\tau}, \operatorname{Pr}_{N}^{\vec{\tau}}(\varphi \mid K B)$ and $\operatorname{Pr}_{N}^{\vec{\tau}}\left(\varphi \mid K B^{\prime}\right)$ are equal. Therefore, the limits are also equal.

Proposition 5.2: If $K B \sim_{r w} \theta$, then $\operatorname{Pr}_{\infty}(\varphi \mid K B)=\operatorname{Pr}_{\infty}(\varphi \mid K B \wedge \theta)$ for any $\varphi$.

Proof: Fix $N$ and $\vec{\tau}$. Then, by the standard properties of conditional probability, we get

$$
\operatorname{Pr}_{N}^{\vec{\tau}}(\varphi \mid K B)=\operatorname{Pr}_{N}^{\vec{\tau}}(\varphi \mid K B \wedge \theta) \cdot \operatorname{Pr}_{N}^{\vec{\tau}}(\theta \mid K B)+\operatorname{Pr}_{N}^{\vec{\tau}}(\varphi \mid K B \wedge \neg \theta) \cdot \operatorname{Pr}_{N}^{\vec{\tau}}(\neg \theta \mid K B) .
$$

By assumption, $\operatorname{Pr}_{N}^{\vec{\tau}}(\theta \mid K B)$ tends to 1 when we take limits, so the first summand tends to $\operatorname{Pr}_{\infty}(\varphi \mid K B \wedge \theta)$. Since $\operatorname{Pr}_{N}^{\vec{\tau}}(\neg \theta \mid K B)$ has limit 0 and $\operatorname{Pr}_{N}^{\vec{\tau}}(\varphi \mid K B \wedge \neg \theta)$ is bounded, the second summand tends to 0 . The result follows.

Theorem 5.3: The relation $\sim_{r w}$ satisfies the properties of And, Cautious Monotonicity, Cut, Left Logical Equivalence, Or, Reflexivity, and Right Weakening.

\section{Proof:}

And: As we mentioned in Section 3.2, this follows from the other properties proved below.

Cautious Monotonicity and Cut: These follow immediately from Proposition 5.2.

Left Logical Equivalence: Follows immediately from Proposition 5.1.

Or: Assume $\operatorname{Pr}_{\infty}(\varphi \mid K B)=\operatorname{Pr}_{\infty}\left(\varphi \mid K B^{\prime}\right)=1$, so that $\operatorname{Pr}_{\infty}(\neg \varphi \mid K B)=\operatorname{Pr}_{\infty}\left(\neg \varphi \mid K B^{\prime}\right)=0$. Fix $N$ and $\vec{\tau}$. Then

$$
\begin{aligned}
\operatorname{Pr}_{N}^{\vec{\tau}}\left(\neg \varphi \mid K B \vee K B^{\prime}\right) & =\operatorname{Pr}_{N}^{\vec{\tau}}\left(\neg \varphi \wedge\left(K B \vee K B^{\prime}\right) \mid K B \vee K B^{\prime}\right) \\
& \leq \operatorname{Pr}_{N}^{\vec{\tau}}\left(\neg \varphi \wedge K B \mid K B \vee K B^{\prime}\right)+\operatorname{Pr}_{N}^{\vec{\tau}}\left(\neg \varphi \wedge K B^{\prime} \mid K B \vee K B^{\prime}\right) \\
& \leq \operatorname{Pr}_{N}^{\vec{\tau}}(\neg \varphi \mid K B)+\operatorname{Pr}_{N}^{\vec{\tau}}\left(\neg \varphi \mid K B^{\prime}\right) .
\end{aligned}
$$

Taking limits, we conclude that $\operatorname{Pr}_{\infty}\left(\neg \varphi \mid K B \vee K B^{\prime}\right)=0$. It follows that $\left(K B \vee K B^{\prime}\right) \sim_{r w} \varphi$.

\footnotetext{
${ }^{10} \operatorname{By} \operatorname{Pr}_{\infty}(\varphi \mid K B)=\operatorname{Pr}_{\infty}\left(\varphi \mid K B^{\prime}\right)$ we mean that either both degrees of belief exist and have the same value, or neither exists. Proposition 5.2 should be interpreted analogously.
} 
Reflexivity: Because we restrict our attention to $K B$ 's that are eventually consistent, $\operatorname{Pr}_{\infty}(K B \mid K B)$ is well-defined. But then $\operatorname{Pr}_{\infty}(K B \mid K B)$ is clearly equal to 1 .

Right Weakening: Suppose $\operatorname{Pr}_{\infty}(\varphi \mid K B)=1$. If $\models \varphi \Rightarrow \varphi^{\prime}$, then the set of worlds satisfying $\varphi^{\prime}$ is a superset of the set of worlds satisfying $\varphi$. Therefore, for any $N$ and $\vec{\tau}, \operatorname{Pr}_{N}^{\vec{\tau}}\left(\varphi^{\prime} \mid K B\right) \geq$ $\operatorname{Pr}_{N}^{\vec{\tau}}(\varphi \mid K B)$. Taking limits, we obtain that

$$
1 \geq \operatorname{Pr}_{\infty}\left(\varphi^{\prime} \mid K B\right) \geq \operatorname{Pr}_{\infty}(\varphi \mid K B)=1,
$$

and so necessarily $\operatorname{Pr}_{\infty}\left(\varphi^{\prime} \mid K B\right)=1$.

Besides demonstrating that $\sim_{r w}$ satisfies the minimal standards of reasonableness for a default inference relation, these properties, particularly the stronger form of Cut and Cautious Monotonicity proved in Proposition 5.2, will prove quite useful in computing degrees of belief, especially when combined with some other properties we prove below (see also Section 7.4). In particular, many of our later results show how random-worlds behaves for knowledge bases and queries that have certain restricted forms. Sometimes a $K B$ that does not satisfy these requirements can be changed into one that does, simply by extending $K B$ with some of its default conclusions. We then appeal to Proposition 5.2 to justify using the new knowledge base instead of the old one. The other rules are also useful, as shown in the following analysis of Poole's "broken-arm" example [Poo89].

Example 5.4: Suppose we have predicates LeftUsable, LeftBroken, RightUsable, RightBroken, indicating, respectively, that the left arm is usable, the left arm is broken, the right arm is usable, and the right arm is broken. Let $K B_{\text {arm }}^{\prime}$ consist of the statements

- $\|\operatorname{LeftUsable}(x)\|_{x} \approx_{1} 1$, $\|\operatorname{LeftUsable}(x) \mid \operatorname{LeftBroken}(x)\|_{x} \approx_{2} 0$ (left arms are typically usable, but not if they are broken),

- $\|$ RightUsable $(x)\left\|_{x} \approx_{3} 1,\right\|$ RightUsable $(x) \mid$ RightBroken $(x) \|_{x} \approx_{4} 0$ (right arms are typically usable, but not if they are broken).

Now, consider $K B_{\text {arm }}=\left(K B_{\text {arm }}^{\prime} \wedge(\operatorname{LeftBroken}(\right.$ Eric $) \vee \operatorname{RightBroken}($ Eric $\left.))\right)$; that is, we know that Eric has a broken arm. Poole observes that if we use Reiter's default logic, there is precisely one extension of $K B_{\text {arm }}$, and in that extension, both arms are usable. However, it can be shown that $K B_{\text {arm }}^{\prime} \wedge$ LeftBroken $($ Eric $) \sim_{r w} \neg$ LeftUsable(Eric) (see Theorem 5.6 below) and hence (using Right Weakening) that $K B_{\text {arm }}^{\prime} \wedge$ LeftBroken $($ Eric $) \sim_{r w} \neg$ LeftUsable $($ Eric $) \vee$ $\neg$ RightUsable(Eric); the same conclusion is obtained from $K B_{\text {arm }}^{\prime} \wedge$ RightBroken(Eric). By the $O r$ rule, it follows that $K B_{\text {arm }} \sim_{r w} \neg$ LeftUsable(Eric) $\vee \neg$ RightUsable(Eric). Using similar reasoning, we can also show that $K B_{\text {arm }} \sim_{r w}$ LeftUsable(Eric) $\vee$ RightUsable(Eric). By applying the $A n d$ rule, we conclude by default from $K B_{\text {arm }}$ that exactly one of Eric's arms is usable, but we draw no conclusions as to which one it is.

The final property mentioned in Section 3.2 is Rational Monotonicity. Recall that Rational Monotonicity asserts that if $K B \sim_{r w} \varphi$ and $K B \not \psi_{r w} \neg \theta$ then $(K B \wedge \theta) \sim_{r w} \varphi$. Random worlds satisfies a weakened form of Rational Monotonicity. In particular, it satisfies Rational Monotonicity except in those situations where limits fail to exist. ${ }^{11}$ If $\operatorname{Pr}_{\infty}(\varphi \mid K B \wedge \theta)$ does exist it

\footnotetext{
${ }^{11}$ As we discuss later in Section 5.3 there are often intuitive reasons for the non-existence of limits.
} 
must be equal to 1 , i.e., we must have $(K B \wedge \theta) \sim_{r w} \varphi$ as desired. Sometimes, however, this limit does not exist. Note that the assumption that $K B \sim_{r w} \varphi$ entails that $\operatorname{Pr}_{\infty}(\varphi \mid K B)$ exists. But Rational Monotonicity's other assumption, that $K B \not \chi_{r w} \neg \theta$ holds if either $\operatorname{Pr}_{\infty}(\theta \mid K B)$ has a value less than one or if this degree of belief does not exist. It is the latter "incompatibility" of $\theta$ with $K B$ that is a potential source of problems. In this case the combination of $K B$ and $\theta$ may fail to assign a limiting degree of belief to $\varphi$ even though $K B$ by itself did. The following theorem summarizes the status of Rational Monotonicity in the random-worlds approach.

Theorem 5.5: Assume that $K B \sim_{r w} \varphi$ and $K B \nvdash_{r w} \neg \theta$. Then $K B \wedge \theta \sim_{r w} \varphi$ provided that $\operatorname{Pr}_{\infty}(\varphi \mid K B \wedge \theta)$ exists. Moreover, a sufficient condition for $\operatorname{Pr}_{\infty}(\varphi \mid K B \wedge \theta)$ to exist is that $\operatorname{Pr}_{\infty}(\theta \mid K B)$ exists.

Proof: Longer proofs, including the proof of this result, are in the appendix.

\subsection{Specificity and inheritance in random worlds}

One way of using random worlds is to derive conclusions about particular individuals, based on general statistical knowledge. This is, of course, the type of reasoning reference-class theories were designed to deal with. Recall, these theories aim to discover a single piece of data-the statistics for a single reference class - that summarizes all the relevant information. This idea is also useful in default reasoning, where we sometimes want to find a single appropriate default. Random worlds rejects this idea as a general approach, but supports it as a valuable heuristic in special cases.

In this section, we give two theorems covering some of the cases where random worlds agrees with the basic philosophy of reference classes. Both results concern specificity - the idea of using the "smallest" relevant reference class for which we have statistics. However, both results also allow some indifference to irrelevant information. In particular, the second theorem also covers certain forms of inheritance (as described in Section 3.3). The results cover almost all of the noncontroversial applications of specificity and inheritance that we are aware of, and do not seem to suffer from any of the commonly found problems such as the disjunctive reference class problem (see Section 2.2). Because our theorems are derived properties rather than postulates, consistency is assured and there are no ad hoc syntactic restrictions on the choice of possible reference classes. We remark that Shastri [Sha89] has also observed that irrelevance properties hold in his framework.

Our first, and simpler, result is basic direct inference, where we have a single reference class that is precisely the "right one". That is, assume that the assertion $\psi(c)$ represents everything the knowledge base tells us about the constant $c$. In this case, we can view the class defined by $\psi(x)$ as the class of all individuals who are "just like $c$ ". If we have adequate statistics for the class $\psi(x)$, then we should clearly use this information. For example, assume that all we know about Eric is that he exhibits jaundice, and let $\psi$ represent the class of patients with jaundice. If we know that $80 \%$ of patients with jaundice exhibit hepatitis, then basic direct inference would dictate a degree of belief of 0.8 in Eric having hepatitis. We would, in fact, like this to hold regardless of any other information we might have in the knowledge base. For example, we may know the proportion of hepatitis among patients in general, or that patients with jaundice 
and fever typically have hepatitis. But if all we know about Eric is that he has jaundice, we would still like to use the statistics for the class of patients with jaundice, regardless of the additional information.

Our result essentially asserts the following: "If we are interested in obtaining a degree of belief in $\varphi(c)$, and the $K B$ is of the form $\psi(c) \wedge\|\varphi(x) \mid \psi(x)\|_{x} \approx \alpha \wedge K B^{\prime}$, then conclude that $\operatorname{Pr}_{\infty}(\varphi(c) \mid K B)=\alpha . "$ (Here, $K B^{\prime}$ is simply intended to denote the rest of $K B$, whatever it may be.) Clearly, in order for the result to hold, we must make certain assumptions. The assumptions we consider can be viewed as ensuring that $\psi(c)$ represents all the information we have about $c$. First, for obvious reasons, we require that $K B^{\prime}$ does not mention $c$. However, this is not enough; we also need to assume that $c$ does not appear in either $\varphi(x)$ or $\psi(x)$. To understand why $c$ cannot appear in $\varphi(x)$, suppose that $\varphi(x)$ is $Q(x) \vee x=c, \psi(x)$ is true, and the $K B$ is $\| \varphi(x) \mid$ true $_{x} \approx_{1}$ 0.5. If the result held in this case, we would erroneously conclude that $\operatorname{Pr}_{\infty}(\varphi(c) \mid K B)=0.5$. But since $\varphi(c)$ holds tautologically, we actually obtain $\operatorname{Pr}_{\infty}(\varphi(c) \mid K B)=1$. To see why the constant $c$ cannot appear in $\psi(x)$, suppose that $\psi(x)$ is $(P(x) \wedge x \neq c) \vee \neg P(x), \varphi(x)$ is $P(x)$, and the $K B$ is $\psi(c) \wedge\|P(x) \mid \psi(x)\|_{x} \approx_{2} 0.5$. Again, if the result held, we would be able to conclude that $\operatorname{Pr}_{\infty}(P(c) \mid K B)=0.5$. But $\psi(c)$ is equivalent to $\neg P(c)$, so in fact $\operatorname{Pr}_{\infty}(P(c) \mid K B)=0$.

As we now show, these assumptions suffice to guarantee the desired result. In fact, the theorem generalizes the basic principle to properties and classes dealing with more than one individual at a time (as is demonstrated in some of the examples following the theorem). In the following, let $\vec{x}=\left\{x_{1}, \ldots, x_{k}\right\}$ and $\vec{c}=\left\{c_{1}, \ldots, c_{k}\right\}$ be sets of distinct variables and distinct constants, respectively. Furthermore, we use $\varphi(\vec{x})$ to indicate that all of the free variables in the formula $\varphi$ are in $\vec{x}$, and we use $\varphi(\vec{c})$ to denote the new formula formed by substituting each $x_{i}$ by $c_{i}$ in $\varphi$. Note that $\varphi$ may contain other constants not among the $c_{i}$ 's; these are unaffected by the substitution.

Theorem 5.6: Let $K B$ be a knowledge base of the form $\psi(\vec{c}) \wedge K B^{\prime}$, and assume that for all sufficiently small tolerance vectors $\vec{\tau}$,

$$
K B[\vec{\tau}] \models\|\varphi(\vec{x}) \mid \psi(\vec{x})\|_{\vec{x}} \in[\alpha, \beta] .
$$

If no constant in $\vec{c}$ appears in $K B^{\prime}$, in $\varphi(\vec{x})$, or in $\psi(\vec{x})$, then $\operatorname{Pr}_{\infty}(\varphi(\vec{c}) \mid K B) \in[\alpha, \beta]$, provided the degree of belief exists. ${ }^{12}$

Proof: See the appendix.

Theorem 5.6 refers to any statistical information about $\|\varphi(\vec{x}) \mid \psi(\vec{x})\|_{\vec{x}}$ that can be inferred from the knowledge base. An important special case is when the knowledge base contains the relevant information explicitly.

Corollary 5.7: Let $K B^{\prime}$ be the conjunction

$$
\psi(\vec{c}) \wedge\left(\alpha \preceq_{i}\|\varphi(\vec{x}) \mid \psi(\vec{x})\|_{\vec{x}} \preceq_{j} \beta\right) .
$$

\footnotetext{
${ }^{12}$ The degree of belief may not exist since $\lim _{\vec{\tau} \rightarrow \overrightarrow{0}} \liminf \inf _{N \rightarrow \infty} \operatorname{Pr}_{N}^{\vec{\tau}}(\varphi \mid K B)$ may not be equal to $\lim _{\vec{\tau} \rightarrow \overrightarrow{0}} \lim \sup _{N \rightarrow \infty} \operatorname{Pr}_{N}^{\vec{\tau}}(\varphi \mid K B)$. However, it follows from the proof of the theorem that both these limits lie in the interval $[\alpha, \beta]$. A similar remark holds for many of our later results.
} 
Let $K B$ be a knowledge base of the form $K B^{\prime} \wedge K B^{\prime \prime}$ such that no constant in $\vec{c}$ appears in $K B^{\prime \prime}$, in $\varphi(\vec{x})$, or in $\psi(\vec{x})$. Then, if the degree of belief exists, we have

$$
\operatorname{Pr}_{\infty}(\varphi(\vec{c}) \mid K B) \in[\alpha, \beta]
$$

Proof: Let $\epsilon>0$, and let $\vec{\tau}$ be sufficiently small so that $\tau_{i}, \tau_{j}<\epsilon$. For this $\vec{\tau}$, the formula $\left(\alpha \preceq_{i}\|\varphi(\vec{x}) \mid \psi(\vec{x})\|_{\vec{x}} \preceq_{j} \beta\right)$ implies $\|\varphi(\vec{x}) \mid \psi(\vec{x})\|_{\vec{x}} \in[\alpha-\epsilon, \beta+\epsilon]$. Therefore, by Theorem 5.6, $\operatorname{Pr}_{\infty}(\varphi(\vec{c}) \mid K B) \in[\alpha-\epsilon, \beta+\epsilon]$. But since this holds for any $\epsilon>0$, it is necessarily the case that $\operatorname{Pr}_{\infty}(\varphi(\vec{c}) \mid K B) \in[\alpha, \beta]$.

It is interesting to note one way in which this result diverges from the reference-class paradigm. Suppose we consider a query $\varphi(c)$, and that our knowledge base $K B$ is as in the hypothesis of Corollary 5.7. While we can indeed conclude that $\operatorname{Pr}_{\infty}(\varphi(\vec{c}) \mid K B) \in[\alpha, \beta]$, the exact value of the degree of belief within this interval depends on the other information in the knowledge base. Thus, while random worlds certainly uses the information $\alpha \preceq_{i}\|\varphi(x) \mid \psi(x)\|_{x} \preceq_{j} \beta$, it does not necessarily ignore the rest of the knowledge base altogether. On the other hand, if the interval $[\alpha, \beta]$ is sufficiently small (and, in particular, when $\alpha=\beta$ ), then we may not care exactly where in the interval the degree of belief lies. In this case, we can ignore all the information in $K B^{\prime}$, and use the single piece of "local" information for computing the degree of belief.

We now present a number of examples that demonstrate the behavior of the direct inference result.

Example 5.8: Consider a knowledge base describing the hepatitis example discussed earlier. In the notation of Corollary 5.7:

$$
K B_{\text {hep }}^{\prime}=\operatorname{Jaun}(\operatorname{Eric}) \wedge\|H e p(x) \mid \operatorname{Jaun}(x)\|_{x} \approx_{1} 0.8,
$$

and

$$
K B_{\text {hep }}=K B_{\text {hep }}^{\prime} \wedge\|H e p(x)\|_{x} \preceq_{2} 0.05 \wedge\|H e p(x) \mid \operatorname{Jaun}(x) \wedge \operatorname{Fever}(x)\|_{x} \approx_{2} 1 .
$$

Then $\operatorname{Pr}_{\infty}\left(\right.$ Hep (Eric) $\left.\mid K B_{\text {hep }}\right)=0.8$ as desired; information about other reference classes (whether more general or more specific) is ignored. Other kinds of information are also ignored, for example, information about other individuals. Thus, $\operatorname{Pr}_{\infty}\left(\operatorname{Hep}(\right.$ Eric $) \mid K B_{\text {hep }} \wedge \operatorname{Hep}($ Tom $\left.)\right)=0.8$.

Although it is nothing but an immediate application of Theorem 5.6, it is worth remarking that the principle of Direct Inference for Defaults (Section 3.3) is satisfied by random-worlds:

Corollary 5.9: Suppose KB implies $\|\varphi(\vec{x}) \mid \psi(\vec{x})\|_{\vec{x}} \approx_{i} 1$, and no constant in $\vec{c}$ appears in $K B$, $\varphi$, or $\psi$. Then $\operatorname{Pr}_{\infty}(\varphi(\vec{c}) \mid K B \wedge \psi(\vec{c}))=1$.

As discussed in Section 3.3, this shows that simple forms of reasoning about classification hierarchies are possible.

Example 5.10: The knowledge base $K B_{f l y}$ from Section 3.3 is, under our interpretation of defaults:

$$
\left\|\operatorname{Fly}(x)\left|\operatorname{Bird}(x)\left\|_{x} \approx_{1} 1 \wedge\right\| F l y(x)\right| \operatorname{Penguin}(x)\right\|_{x} \approx_{2} 0 \wedge \forall x(\operatorname{Penguin}(x) \Rightarrow \operatorname{Bird}(x)) .
$$

$\operatorname{Then} \operatorname{Pr}_{\infty}\left(\right.$ Fly $($ Tweety $) \mid K B_{\text {fly }} \wedge$ Penguin $($ Tweety $\left.)\right)=0$. That is, we conclude that Tweety the penguin does not fly, even though he is also a bird and birds generally do fly. 
Given this preference for the most specific reference class, one might wonder why random worlds does not encounter the problem of disjunctive reference classes (see Section 2.2). The following example, based on the example from Section 2.2, provides one answer.

Example 5.11: Recall the knowledge base $K B_{h e p}^{\prime}$ from the hepatitis example above, and consider the disjunctive reference class $\psi(x)={ }_{\text {def }} \operatorname{Jaun}(x) \wedge(\neg H e p(x) \vee x=$ Eric $)$. Clearly, as the domain size grows large, $\|H e p(x) \mid \psi(x)\|_{x}$ becomes arbitrarily close to $0 .{ }^{13}$ Therefore, for any fixed $\epsilon>0$

$$
\operatorname{Pr}_{\infty}\left(\left\|H e p(x)\left|\psi(x) \|_{x} \in[0, \epsilon]\right| K B_{h e p}^{\prime}\right)=1 .\right.
$$

We can construct a new knowledge base $K B_{\vee h e p}=K B_{h e p}^{\prime} \wedge\|H e p(x) \mid \psi(x)\|_{x} \in[0, \epsilon]$. Furthermore, $K B_{\vee h e p} \models \psi(E$ ric $)$. Hence, $K B_{\vee h e p}$ contains a more specific reference class for $H e p(E r i c)$ than $\operatorname{Jaun}(x)$ with very different statistics. Yet, by Proposition 5.2, we know that $\operatorname{Pr}_{\infty}\left(H e p(\right.$ Eric $\left.) \mid K B_{h e p}^{\prime}\right)=\operatorname{Pr}_{\infty}\left(H e p(\right.$ Eric $\left.) \mid K B_{\vee h e p}\right)$, and in Example 5.8 we showed this to be equal to 0.8. So random worlds avoids using the spurious disjunctive class $\psi(x)$ even in a knowledge base that explicitly includes statistics from this class. Theorem 5.6 does not apply here because the class $\psi(x)$ explicitly mentions the constant Eric. Another way of seeing that the class $\psi(x)$ does not affect the random-worlds computation is to observe that its statistics are not informative, i.e., these statistics are true in almost all worlds. Hence $\psi(x)$ 's statistics places no constraints on the sets of worlds that determine the degree of belief. As we shall see in Example 5.22, when we do have informative statistics for a class, those statistics can be used, even if the class is disjunctive.

As we have said, we are not limited to unary predicates, nor to examining only one individual at a time.

Example 5.12: In Example 4.4, we showed how to formalize the elephant-zookeeper example discussed in Section 3.4. As we now show, the natural representation of $K B_{\text {likes }}$ indeed yields the answers we expect. We consider two queries. First, assume that we are interested in finding out whether Clyde likes Eric. In this case, we can use the class of pairs $\psi(x, y)=$ Elephant $(x) \wedge$ Zookeeper $(y)$. Applying Corollary 5.9 to the first default in $K B_{\text {likes }}$, we can conclude that $\operatorname{Pr}_{\infty}\left(\right.$ Likes $($ Clyde, Eric $\left.) \mid K B_{\text {likes }}\right)=1$. Second, we examine whether or not Clyde likes Fred. Applying Corollary 5.9 to the second default in $K B_{\text {likes }}$, we can conclude that $\operatorname{Pr}_{\infty}\left(\right.$ Likes $($ Clyde, Fred $\left.) \mid K B_{\text {likes }}\right)=0$. Note that we cannot apply Corollary 5.9 to the first default in $K B_{\text {likes }}$ to conclude that Clyde likes Fred. The conditions of the corollary are violated, because the constant Fred is used elsewhere in the knowledge base.

The same principles continue to hold for more complex sentences; for example, we can mix first-order logic and statistical knowledge arbitrarily and we can nest defaults.

Example 5.13: In Example 4.5, we showed how to express the default: "People who have at least one tall parent are typically tall." If we have this default, and also know that $\exists y(\operatorname{Child}($ Alice, $y) \wedge \operatorname{Tall}(y))$ (Alice has a tall parent), Corollary 5.9 tells us that we can conclude by default that Tall(Alice).

\footnotetext{
${ }^{13}$ This actually relies on the fact that, with high probability, the proportion (as the domain size grows) of jaundiced patients without hepatitis is nonzero. We do not prove this fact here; see [PV89, GHK96a].
} 
Example 5.14: In Example 4.6, we showed how the default "Typically, people who normally go to bed late normally rise late" can be expressed in our language using the knowledge base $K B_{\text {late. }}$ Let $K B_{\text {late }}^{\prime}$ be

$$
K B_{\text {late }} \wedge \| \text { To-bed-late }\left(\text { Alice, }, y^{\prime}\right) \mid \operatorname{Day}\left(y^{\prime}\right) \|_{y^{\prime}} \approx_{2} 1 .
$$

By Corollary 5.9, Alice typically rises late. That is,

$$
\operatorname{Pr}_{\infty}\left(\| \text { Rises-late }(\text { Alice, } y)\left|\operatorname{Day}(y) \|_{y} \approx_{1} 1\right| K B_{\text {late }}^{\prime}\right)=1 .
$$

By Cautious Monotonicity and Cut, we can add this conclusion (which is itself a default) to $K B_{\text {late }}^{\prime}$. By Corollary 5.9 again, we then conclude that Alice can be expected to rise late on any particular day, say Tomorrow. So, for instance:

$$
\operatorname{Pr}_{\infty}\left(\text { Rises-late }(\text { Alice }, \text { Tomorrow }) \mid K B_{\text {late }}^{\prime} \wedge \text { Day }(\text { Tomorrow })\right)=1 \text {. }
$$

In all the examples presented so far in this section, we have statistics for precisely the right reference class to match our knowledge about the individual(s) in question; Theorem 5.6 and its corollaries require this. Unfortunately, in many cases our statistical information is not detailed enough for Theorem 5.6 to apply. Consider the knowledge base $K B_{\text {hep }}$ from the hepatitis example. Here we have statistics for the occurrence of hepatitis among the class of patients who are just like Eric, so we can use these to induce a degree of belief in Hep(Eric). But now consider the knowledge base $K B_{\text {hep }} \wedge \operatorname{Tall}(E r i c)$. Since we do not have statistics for the frequency of hepatitis among tall patients, the results we have seen so far do not apply. We would like to be able to ignore Tall(Eric). But what entitles us to ignore Tall(Eric) and not Jaun(Eric)? To solve this problem in complete generality requires a better theory of irrelevance than we currently have. Nevertheless, our next theorem covers many cases, including many of the less controversial examples found in the default reasoning literature.

The theorem we present deals with a knowledge base $K B$ that defines a "minimal" reference class $\psi_{0}$ with respect to the query $\varphi(c)$. More precisely, assume that $K B$ gives statistical information regarding $\left\|\varphi(x) \mid \psi_{i}(x)\right\|_{x}$ for a number of different classes $\psi_{i}(x)$. Further suppose that, among these classes, there is one class $\psi_{0}(x)$ that is minimal - all other classes are strictly larger or entirely disjoint from it. Our result states that if we also know $\psi_{0}(c)$, we can use the statistics for $\left\|\varphi(x) \mid \psi_{0}(x)\right\|_{x}$ to induce a degree of belief in $\varphi(c)$. What makes this such an interesting result is that we are allowed to know more about $c$ than just $\psi_{0}(c)$; any extra information will be treated as being irrelevant. This pattern of reasoning is best explained using an example:

Example 5.15: Assume we have a knowledge base $K B_{\text {taxonomy }}$ containing information about birds and animals; in particular, $K B_{\text {taxonomy }}$ contains a taxonomic hierarchy of this domain. Moreover, $K B_{\text {taxonomy }}$ contains the following information about the swimming ability of various types of animals:

$\begin{array}{llll}\|\operatorname{Swims}(x) \mid \operatorname{Penguin}(x)\|_{x} & \approx_{1} & 0.9 & \wedge \\ \|\operatorname{Swims}(x) \mid \operatorname{Sparrow}(x)\|_{x} & \approx_{2} & 0.01 & \wedge \\ \|\operatorname{Swims}(x) \mid \operatorname{Bird}(x)\|_{x} & \approx_{3} & 0.05 & \wedge \\ \|\operatorname{Swims}(x) \mid \operatorname{Animal}(x)\|_{x} & \approx_{4} & 0.3 & \wedge \\ \|\operatorname{Swims}(x) \mid \operatorname{FiSh}(x)\|_{x} & \approx_{5} & 1 . & \end{array}$


If we also know that Opus is a penguin, then in order to determine whether Opus swims the best reference class is surely the class of penguins. The remaining classes are either larger (in the case of birds or animals), or disjoint (in the case of sparrows and fish). This is the case even if we know that Opus is a black penguin with a large nose. That is, Opus inherits the statistics for the minimal class $\psi_{0}$ - penguins - even though the class of individuals just like Opus is smaller than $\psi_{0}$.

That random-worlds validates this intuition is formalized in the next theorem. This theorem requires that no symbol in $\varphi(x)$ appear in the knowledge base other than in statistics of the form $\|\varphi(x) \mid \psi(x)\|_{x}$ for various $\psi(x)$. This is necessary for our assumption of a unique minimal reference class to be a practical one. Suppose that, in violation of this condition, the knowledge base contains $\forall x(\psi(x) \Rightarrow \varphi(x))$. Clearly $\psi(x)$ is in fact a reference class for $\varphi(x)$ (where the statistic is $100 \%$ ). But if we identify reference classes only by looking for terms of the form $\|\varphi(x) \mid \psi(x)\|_{x}$, we will not notice this. Obviously the minimality assumption needs to consider all reference classes, irrespective of syntactic form. But because first-order logic provides many subtle and nonobvious ways to constrain statistics relating to $\varphi(x)$, we simplify the issue by assuming that the only mention of information that might be related to $\varphi(x)$ is contained in explicit statistical assertions. Of course, it would be very interesting to find a result that addresses cases in which this assumption is not true.

Theorem 5.16: Let $c$ be a constant and let $K B$ be a knowledge base satisfying the following conditions:

(a) $K B \models \psi_{0}(c)$,

(b) for any expression of the form $\|\varphi(x) \mid \psi(x)\|_{x}$ in $K B$, it is the case that either $K B \models$ $\forall x\left(\psi_{0}(x) \Rightarrow \psi(x)\right)$ or that $K B \models \forall x\left(\psi_{0}(x) \Rightarrow \neg \psi(x)\right)$,

(c) the (predicate, function, and constant) symbols in $\varphi(x)$ appear in $K B$ only on the left-hand side of the conditionals in the proportion expressions described in condition (b),

(d) the constant $c$ does not appear in the formula $\varphi(x)$.

Assume that for all sufficiently small tolerance vectors $\vec{\tau}$ :

$$
K B[\vec{\tau}] \models\left\|\varphi(x) \mid \psi_{0}(x)\right\|_{x} \in[\alpha, \beta] .
$$

Then $\operatorname{Pr}_{\infty}(\varphi(c) \mid K B) \in[\alpha, \beta]$, provided the degree of belief exists.

Proof: See the appendix.

Again, the following analogue to Corollary 5.7 is immediate:

Corollary 5.17: Let $K B^{\prime}$ be the conjunction

$$
\psi_{0}(c) \wedge\left(\alpha \preceq_{i}\left\|\varphi(x) \mid \psi_{0}(x)\right\|_{x} \preceq_{j} \beta\right) .
$$

Let $K B$ be a knowledge base of the form $K B^{\prime} \wedge K B^{\prime \prime}$ that satisfies conditions (b), (c), and (d) of Theorem 5.16. Then, if the degree of belief exists,

$$
\operatorname{Pr}_{\infty}(\varphi(c) \mid K B) \in[\alpha, \beta]
$$


This theorem and corollary have many useful applications.

Example 5.18: Consider the knowledge bases $K B_{h e p}^{\prime}$ and $K B_{h e p}$ concerning jaundice and hepatitis from Example 5.8. In that example, we supposed that the only information about Eric contained in the knowledge base was that Eric has jaundice. It is clearly more realistic to assume that Eric's hospital records contain more information than just this fact. Theorem 5.16 allows us to ignore this information in a large number of cases.

For example,

$$
\operatorname{Pr}_{\infty}\left(\text { Hep }(\text { Eric }) \mid K B_{h e p}^{\prime} \wedge \text { Fever }(\text { Eric }) \wedge \operatorname{Tall}(\text { Eric })\right)=0.8
$$

as desired. On the other hand,

$$
\operatorname{Pr}_{\infty}\left(\text { Hep }(\text { Eric }) \mid K B_{\text {hep }} \wedge \text { Fever }(\text { Eric }) \wedge \operatorname{Tall}(\text { Eric })\right)=1 .
$$

(Recall that $K B_{\text {hep }}$ includes $\|H e p(x) \mid \operatorname{Jaun}(x) \wedge \operatorname{Fever}(x)\|_{x} \approx_{2} 1$, while $K B_{h e p}^{\prime}$ does not.) This shows why it is important that we identify the most specific reference class for the query $\varphi$. The most specific reference statistic for $\operatorname{Hep}\left(\right.$ Eric) with respect to $K B_{\text {hep }}^{\prime} \wedge$ Fever $($ Eric $) \wedge \operatorname{Tall}($ Eric $)$ is $\|$ Hep $(x) \mid \operatorname{Jaun}(x) \|_{x} \approx_{1}$ 0.8, while with respect to $K B_{\text {hep }} \wedge \operatorname{Fever}($ Eric $) \wedge \operatorname{Tall}($ Eric $)$ it is $\|\operatorname{Hep}(x) \mid \operatorname{Jaun}(x) \wedge \operatorname{Fever}(x)\|_{x} \approx_{2} 1$. In the latter case, the less-specific reference classes Jaun and true are ignored, and in both cases Theorem 5.16 allows us to ignore the extra information Tall(Eric). Note that the theorem does not allow us to conclude that

$$
\operatorname{Pr}_{\infty}\left(\text { Hep }(\text { Eric }) \mid K B_{\text {hep }} \wedge \operatorname{Tall}(\text { Eric })\right)=0.8
$$

The class Jaun is no longer the unique most specific reference class, since we also have statistics for the more specific class Jaun $\wedge$ Fever. Nevertheless, this conclusion is, in fact, reached by random worlds.

As discussed in Section 3.3, various inheritance properties are considered desirable in default reasoning as well. To begin with, we note that Theorem 5.16 covers the simpler cases (which can also be seen as applications of rational monotonicity):

Example 5.19: In simple cases, Theorem 5.16 shows that random worlds is able to apply defaults in the presence of "obviously irrelevant" additional information. For example, using the knowledge base $K B_{f l y}$ (see Example 5.10):

$$
\operatorname{Pr}_{\infty}\left(\text { Fly }(\text { Tweety }) \mid K B_{\text {fly }}^{\prime} \wedge \text { Penguin }(\text { Tweety }) \wedge \text { Yellow }(\text { Tweety })\right)=0 .
$$

That is, Tweety the yellow penguin is still not able to fly.

Theorem 5.16 also validates more difficult reasoning patterns that have caused problems for many default reasoning theories. In particular, we validate exceptional-subclass inheritance, in which a class that is exceptional in one respect can nevertheless inherit other unrelated properties: 
Example 5.20: If we consider the property of warm-bloodedness as well as flight, we get:

$$
\operatorname{Pr}_{\infty}\left(\begin{array}{l|l}
\text { Warm-blooded }(\text { Tweety }) & \left.\begin{array}{l}
\text { KB fly } \wedge \text { Penguin }(\text { Tweety }) \wedge \\
\| \text { Warm-blooded }(x) \mid \operatorname{Bird}(x) \|_{x} \approx_{3} 1
\end{array}\right)=1 .
\end{array}\right.
$$

Knowing that Tweety does not fly because he is a penguin does not prevent us from assuming that he is like typical birds in other respects.

The drowning-problem variant of the exceptional-subclass inheritance problem is also covered by the theorem.

Example 5.21: Suppose we know, as in Section 3.3, that yellow things tend to be highly visible. Then:

$$
\operatorname{Pr}_{\infty}\left(\begin{array}{l|l}
\text { Easy-to-see }(\text { Tweety }) & \begin{array}{l}
K B_{\text {fly }} \wedge \text { Penguin }(\text { Tweety }) \wedge \text { Yellow }(\text { Tweety }) \wedge \\
\| \text { Easy-to-see }(x) \mid \text { Yellow }(x) \|_{x} \approx_{3} 1
\end{array}
\end{array}\right)=1
$$

Here, all that matters is that Tweety is a yellow object. The fact that he is a bird, and an exceptional bird at that, is rightly ignored.

Notice that, unlike Theorem 5.6, the conditions of Theorem 5.16 do not extend to inferring degrees of belief in $\varphi(\vec{c})$, where $\vec{c}$ is a tuple of constants. Roughly speaking, the reason lies in the ability of the language to create connections between different constants in the tuple. For example, let $K B^{\prime}$ be $\|H e p(x) \wedge \neg H e p(y)\|_{x, y} \approx_{1}$ 0.2. By Theorem 5.6 (taking $\psi_{0}\left(x_{1}, x_{2}\right)$ to be true $), \operatorname{Pr}_{\infty}\left(H e p(\right.$ Tom $) \wedge \neg H e p($ Eric $\left.) \mid K B^{\prime}\right)=0.2$. But, of course, $\operatorname{Pr}_{\infty}(\operatorname{Hep}($ Tom $) \wedge$ $\neg H e p($ Eric $) \mid K B^{\prime} \wedge$ Tom $=$ Eric $)=0$. The additional information regarding Tom and Eric cannot be ignored. A version of Theorem 5.16 where we replaced $c$ by $\vec{c}$ would incorrectly attempt to ignore this information. This example might suggest that this is a problem related only to the use of equality, but more complex examples that do not mention equality can also be constructed.

As a final example in this section, we revisit the issue of disjunctive reference classes. As we saw in Example 5.11, random worlds does not suffer from the "disjunctive reference class" problem. In Section 2.2, we observed that some systems avoid this problem by simply outlawing disjunctive reference classes, which is problematic, as such classes are sometimes useful. The next example demonstrates that random worlds does, in fact, treat disjunctive reference classes appropriately.

Example 5.22: Recall that in Section 2.2 we gave an example involving disjunctive reference classes for Tay-Sachs disease. The corresponding statistical information was represented, in our framework, as the knowledge base $K B$ :

$$
\|T S(x) \mid E E J(x) \vee F C(x)\|_{x} \approx_{1} 0.02
$$

Given a baby Eric of eastern-European extraction, Theorem 5.16 shows us that

$$
\operatorname{Pr}_{\infty}(T S(\text { Eric }) \mid K B \wedge \operatorname{EEJ}(\text { Eric }))=0.02 .
$$


That is, random worlds is able to use the information derived from the disjunctive reference class, and apply it to an individual known to be in the class; indeed, through inheritance it also deals with the case where we have additional information determining to which of the two populations this specific individual belongs. Thus, disjunctive reference classes are treated in the same manner as other potential reference classes.

The type of specificity and inheritance reasoning covered by the results in this section are special cases of general inheritance reasoning. While these theorems show that random worlds does support many noncontroversial instances of such reasoning, proving a more general theorem asserting this claim is surprisingly subtle (partly because of the existence of numerous divergent semantics and intuitions for inheritance reasoning [THT87]). We are currently working towards stating and proving such a general claim, for the case in which we have an inheritance hierarchy of defaults and universal implications. On the other hand, it is easy to see that random worlds does not validate general inheritance reasoning in an arbitrary statistical context (i.e., where some statistical values are less than 1 , and so do not state defaults). We discuss why this happens below, in Example 5.25, and argue that we should not want simple inheritance in all contexts anyway.

\subsection{Competing reference classes}

In previous sections we have always been careful to consider examples in which there was an obviously "best" reference class. In practice, we will not always be this fortunate. Referenceclass theories usually cannot give useful answers when there are competing candidates for the best class. However, random worlds does not have this problem, because the degrees of belief it defines can be combinations of the values for competing classes. In this section we examine, in very general terms, three types of competing information. The first concerns conflicts between specificity and accuracy, the second between information that is too specific and information that is too general, and the last between incomparable reference classes, so that the specificity principle is not applicable.

We discussed the conflict between specificity and accuracy in Section 2.3. This problem was noticed by Kyburg, who addresses this issue with his strength rule. In Section 2.3, we argued that, to assign a degree of belief to Chirps (Tweety), we should be able to use the tighter interval $[0.7,0.8]$ even though it is associated with a less specific reference class. As we observed, Kyburg's strength rule attempts to capture this intuition. As the following result shows, the random worlds method also captures this intuition (without requiring any special rules), at least when the reference classes form a chain. ${ }^{14}$

Theorem 5.23: Suppose KB has the form

$$
\bigwedge_{i=1}^{m}\left(\alpha_{i} \preceq_{\ell_{i}}\left\|\varphi(x) \mid \psi_{i}(x)\right\|_{x} \preceq r_{i} \beta_{i}\right) \wedge \psi_{1}(c) \wedge K B^{\prime}
$$

\footnotetext{
${ }^{14}$ Kyburg's rule also applies to cases where the reference classes do not form a chain. The random-worlds method disagrees with the strength rule in these cases. For example, if we know that only $20 \%$ of Republicans are pacifists, that only $20 \%$ of bankers are pacifists, and that Morgan is a Republican banker, Kyburg's strength rule would conclude that our degree of belief that Morgan is a pacifist is 0.2 . On the other hand, the randomworlds method would view this as two pieces of evidence counting against Morgan being a pacifist; it can be shown that this would result in a degree of belief less than 0.2 .
} 
and, for all $i, K B \models \forall x\left(\psi_{i}(x) \Rightarrow \psi_{i+1}(x)\right) \wedge \neg\left(\left\|\psi_{1}(x)\right\|_{x} \approx_{1} 0\right)$. Assume also that no symbol appearing in $\varphi(x)$ appears in $K B^{\prime}$ or in any $\psi_{i}(c)$. Further suppose that, for some $j,\left[\alpha_{j}, \beta_{j}\right]$ is the tightest interval. That is, for all $i \neq j, \alpha_{i}<\alpha_{j}<\beta_{j}<\beta_{i}$. Then, if it exists,

$$
\operatorname{Pr}_{\infty}(\varphi(c) \mid K B) \in\left[\alpha_{j}, \beta_{j}\right]
$$

Proof: See the appendix.

Example 5.24: The example described in Section 2.3 is essentially captured by the following knowledge base $K B_{\text {chirps }}$ :

$$
\begin{aligned}
& 0.7 \preceq_{1}\|\operatorname{Chirps}(x) \mid \operatorname{Bird}(x)\|_{x} \preceq_{2} 0.8 \wedge \\
& 0 \preceq_{3}\|\operatorname{Chirps}(x) \mid \operatorname{Magpie}(x)\|_{x} \preceq_{4} 0.99 \wedge \\
& \forall x(\operatorname{Magpie}(x) \Rightarrow \operatorname{Bird}(x)) \wedge \\
& \text { Magpie }(\text { Tweety }) .
\end{aligned}
$$

It follows from Theorem 5.23 that $\operatorname{Pr}_{\infty}\left(\right.$ Chirps $($ Tweety $\left.) \mid K B_{\text {chirps }}\right) \in[0.7,0.8] .{ }^{15}$

Next, we consider a different way in which competing reference classes can arise: when one reference class is too specific, and the other too general.

Example 5.25: We illustrate the problem with a example based on one of Goodwin's [Goo92]. Consider KB magpie:

$$
\begin{aligned}
& \|\operatorname{Chirps}(x) \mid \operatorname{Bird}(x)\|_{x} \approx_{1} 0.9 \wedge \\
& \|\operatorname{Chirps}(x) \mid \operatorname{Magpie}(x) \wedge \operatorname{Moody}(x)\|_{x} \approx_{2} 0.2 \wedge \\
& \forall x(\operatorname{Magpie}(x) \Rightarrow \operatorname{Bird}(x)) \wedge \\
& \operatorname{Magpie}(\text { Tweety }) .
\end{aligned}
$$

Reference class theories would typically ignore the information about moody magpies: since Tweety is not known to be moody, the class of moody magpies is not even a legitimate reference class in these theories. Using such approaches, the degree of belief would be 0.9. Goodwin argues that this is not completely reasonable - why should we ignore the information about moody magpies? Tweety could be moody (the knowledge base leaves the question open). In fact, it is consistent with $K B_{\text {magpie }}$ that magpies are generally moody. But ignoring the second statistic in effect amounts to assuming that magpies generally are not moody. It seems hard to see why this is a reasonable assumption. The random-worlds approach supports Goodwin's intuition, and the degree of belief that Tweety flies, given $K B_{\text {magpie }}$, can be shown to have a value which is less than 0.9 .

This example illustrates a general phenomenon: if we do not have a "most appropriate" reference class (in the sense of Theorem 5.6), then random worlds combines information from more specific classes as well as from more general classes. Hence, as we mentioned in the previous section, random worlds does not always validate inheritance reasoning: pure inheritance

\footnotetext{
${ }^{15}$ Strictly speaking, a direct application of Theorem 5.23 would require that $K B_{\text {chirps }}$ contains $\neg\left(\|\operatorname{Magpie}(x)\|_{x} \approx_{i} 0\right)$. But the maximum-entropy techniques of Section 6 can be used to show that this actually follows by default. Hence, by Proposition 5.2, we can consider this to be in $K B_{\text {chirps }}$.
} 
reasoning would always look to superclasses and ignore subclasses. We agree with Goodwin that this property of pure inheritance reasoning can lead to unintuitive conclusions, especially when we are dealing with quantitative information.

The third and most important type of conflict is when we have incomparable reference classes. As we argued in Section 2.3, this case is likely to come up often in practice. While the complete characterization of the behavior of random worlds in such cases would appear to be complex, the following theorem illustrates what happens when the competing references classes are essentially disjoint. We capture "essentially disjoint" here by assuming that the overlap between these classes consists of precisely one member: the individual $c$ addressed in our query. We can generalize the following theorem to the case where we simply assume that the overlap between competing reference classes $\psi$ and $\psi^{\prime}$ is small relative to the sizes of the two classes; that is, where $\left\|\psi(x) \wedge \psi^{\prime}(x) \mid \psi(x)\right\|_{x} \approx 0$ and $\left\|\psi(x) \wedge \psi^{\prime}(x) \mid \psi^{\prime}(x)\right\|_{x} \approx 0$. For simplicity, we omit the details of this extension here.

It turns out that, under this assumption, random worlds provides an independent derivation of a well-known technique for combining evidence: Dempster's rule of combination [Sha76]. Dempster's rule addresses the issue of combining independent pieces of evidence. Consider a query $P(c)$, and assume we have competing reference classes that are all appropriate for this query. In this case, the different pieces of evidence are the proportions of the property $P$ in the different competing reference classes. More precisely, if $\psi_{i}(c)$ holds, we can view the fact that $\left\|P(x) \mid \psi_{i}(x)\right\|_{x} \approx \alpha_{i}$ as giving evidence of weight $\alpha_{i}$ in favor of $P(c)$. The fact that the intersection between the different classes is "small" means that almost disjoint samples were used to compute these pieces of evidence; thus, it is perhaps reasonable to view them as being independent. Under this interpretation, Dempster's rule tells us how to combine the different pieces of evidence to obtain an appropriate degree of belief in $P(c)$. The function used in Dempster's rule is $\delta:[0,1]^{m} \rightarrow[0,1]$, defined as follows:

$$
\delta\left(\alpha_{1}, \ldots, \alpha_{m}\right)=\frac{\prod_{i=1}^{m} \alpha_{i}}{\prod_{i=1}^{m} \alpha_{i}+\prod_{i=1}^{m}\left(1-\alpha_{i}\right)} .
$$

As the following theorem shows, this is also the answer obtained by random worlds. Since $\delta$ is undefined if some $\alpha_{i}$ are equal to 1 while others are equal to 0 , we assume that this is not the case in the theorem.

Theorem 5.26: Let $P$ be a unary predicate, and consider a knowledge base $K B$ of the following form: ${ }^{16}$

$$
\bigwedge_{i=1}^{m}\left(\left\|P(x) \mid \psi_{i}(x)\right\|_{x} \approx_{i} \alpha_{i} \wedge \psi_{i}(c)\right) \wedge \bigwedge_{\substack{i, j=1 \\ i \neq j}}^{m} \exists ! x\left(\psi_{i}(x) \wedge \psi_{j}(x)\right),
$$

where either $\alpha_{i}<1$ for all $i=1, \ldots, m$, or $\alpha_{i}>0$ for all $i=1, \ldots, m$. Then, if neither $P$ nor $c$ appear anywhere in the formulas $\psi_{i}(x)$, then

$$
\operatorname{Pr}_{\infty}(P(c) \mid K B)=\delta\left(\alpha_{1}, \ldots, \alpha_{m}\right) .
$$

Proof: See the appendix.

\footnotetext{
${ }^{16}$ Here, $\exists$ ! $x$ stands for "there exists a unique $x$ such that...".
} 
We illustrate this theorem on what is, perhaps, the most famous example of conflicting information - the Nixon Diamond [RC81]. Suppose we are interested in assigning a degree of belief to the assertion "Nixon is a pacifist". Assume that we know that Nixon is both a Quaker and a Republican, and we have statistical information for the proportion of pacifists within both classes. This is an example where we have two incomparable reference classes for the same query. More formally, assume that $K B_{\text {Nixon }}$ is

$$
\begin{aligned}
& \| \text { Pacifist }(x) \mid \text { Quaker }(x) \|_{x} \approx_{1} \alpha \wedge \\
& \| \text { Pacifist }(x) \mid \text { Republican }(x) \|_{x} \approx_{2} \beta \wedge \\
& \text { Quaker }(\text { Nixon }) \wedge \text { Republican }(\text { Nixon }) \wedge \\
& \exists ! x(\text { Quaker }(x) \wedge \text { Republican }(x)),
\end{aligned}
$$

and that $\varphi$ is Pacifist(Nixon). ${ }^{17}$ The degree of belief $\operatorname{Pr}_{\infty}\left(\varphi \mid K B_{\text {Nixon }}\right)$ takes different values, depending on the values $\alpha$ and $\beta$ for the two reference classes. If $\{\alpha, \beta\} \neq\{0,1\}$, then $\operatorname{Pr}_{\infty}\left(\varphi \mid K B_{\text {Nixon }}\right)$ always exists and its value is equal to $\frac{\alpha \beta}{\alpha \beta+(1-\alpha)(1-\beta)}$. If, for example, $\beta=0.5$, so that the information for Republicans is neutral, we get that $\operatorname{Pr}_{\infty}\left(\varphi \mid K B_{\text {Nixon }}\right)=\alpha$ : the data for Quakers is used to determine the degree of belief. If the evidence given by the two reference classes is conflicting- $\alpha>0.5>\beta$ - then $\operatorname{Pr}_{\infty}\left(\varphi \mid K B_{\text {Nixon }}\right) \in[\alpha, \beta]$ : some intermediate value is chosen. If, on the other hand, the two reference classes provide evidence in the same direction, then the degree of belief is greater than both $\alpha$ and $\beta$. For example, if $\alpha=\beta=0.8$, then the degree of belief would be around 0.94. This has a reasonable explanation: if we have two independent bodies of evidence, both supporting $\varphi$, when we combine them we should get even more support for $\varphi$.

Now, assume that our information is not entirely quantitative. For example, we may know that "Quakers are typically pacifists". In our framework, this corresponds to assigning $\alpha=$ 1. If our information for Republicans is not a default, so that $\beta>0$, then it follows from Theorem 5.26 that $\operatorname{Pr}_{\infty}\left(\varphi \mid K B_{\text {Nixon }}\right)=1$. As expected, a default (i.e., an "extreme" value) dominates. But what happens in the case where we have conflicting defaults for the two reference classes? It turns out that, in this case, the limiting probability does not exist. This is because the limit is non-robust: its value depends on the way in which the tolerances $\vec{\tau}$ tend to 0 . More precisely, if $\tau_{1} \ll \tau_{2}$, so that the "almost all" in the statistical interpretation of the first conjunct is much closer to "all" than the "almost none" in the second conjunct is closer to "none", then the limit is 1 . We can view the magnitude of the tolerance as representing the strength of the default. Thus, in this case, the first conjunct represents a default with higher priority than the second conjunct. Symmetrically, if $\tau_{1} \gg \tau_{2}$, then the limit is 0 . On the other hand, if $\tau_{1}=\tau_{2}$, then the limit is $1 / 2$.

The nonexistence of this limit is not simply a technical artifact of our approach. The fact that we obtain different limiting degrees of belief depending on how $\vec{\tau}$ goes to 0 is closely related to the existence of multiple extensions in many other theories of default reasoning (for instance, in default logic [Rei80]). Both non-robustness and the existence of more than one extension suggest a certain incompleteness of our knowledge. It is well-known that, in the presence of conflicting defaults, we often need more information about the strength of the different defaults in order to resolve the conflict. Our approach has the advantage of pinpointing the type of

\footnotetext{
${ }^{17}$ As pointed out above, Theorem 5.26 can be generalized so that instead of asserting that Nixon is the only Quaker Republican, it is sufficient to assert that there are very few Quaker-Republicans.
} 
information that would suffice to reach a decision. Note that our formalism does give us an explicit way to state in this example that the two extensions are equally likely, by asserting that the defaults that generate them have equal strength; namely, we can use $\approx_{1}$ to capture both default statements, rather than using $\approx_{1}$ and $\approx_{2}$. In this case, we get the answer $1 / 2$, as expected. However, it is not always appropriate to conclude that defaults have equal strength. It is not difficult to extend our language to allow the user to prioritize defaults, by defining the relative sizes of the components $\tau_{i}$ of the tolerance vector.

As we mentioned, Theorem 5.26 tells us only how to combine statistics from competing reference classes in the very special case where the intersection of the different reference classes is small. Shastri [Sha89, pp. 331-332] describes a result in the same spirit, but for a different special case: he assumes that, in addition to the statistics for $P$ within each reference class, the statistics for $P$ in the general population are also known. Shastri's result is based on maximum entropy. Maximum entropy is in fact a very general tool for computing degrees of belief, provided we restrict to knowledge bases that involve only unary predicates and are well-behaved in a sense made precise in [GHK94]. (See the discussion in Section 6.)

\subsection{Independence}

As we have seen so far, random worlds captures many of the natural reasoning heuristics that have been proposed in the literature. Another heuristic is a default assumption that all properties are probabilistically independent unless we know otherwise. Random-worlds captures this principle as well, in many cases. It is, in general, very hard to give simple syntactic tests for when a knowledge base forces two properties to be dependent. The following theorem concerns one very simple scenario where we can be sure that no relationship is forced.

Consider two disjoint vocabularies $\Phi$ and $\Phi^{\prime}$, and two respective knowledge base and query pairs: $K B, \varphi \in \mathcal{L}(\Phi)$, and $K B^{\prime}, \varphi^{\prime} \in \mathcal{L}\left(\Phi^{\prime}\right)$. We can prove that

$$
\operatorname{Pr}_{\infty}\left(\varphi \wedge \varphi^{\prime} \mid K B \wedge K B^{\prime}\right)=\operatorname{Pr}_{\infty}(\varphi \mid K B) \times \operatorname{Pr}_{\infty}\left(\varphi^{\prime} \mid K B^{\prime}\right)
$$

That is, if there is no connection between the symbols in the two vocabularies, the two queries will be independent: the probability of their conjunction is the product of their probabilities. We now prove a slightly more general case, where the two queries are both allowed to refer to some constant $c$.

Theorem 5.27: Let $\Phi_{1}$ and $\Phi_{2}$ be two subvocabularies of $\Phi$ that are disjoint except for the constant c. Consider $K B_{1}, \varphi_{1} \in \mathcal{L}\left(\Phi_{1}\right)$ and $K B_{2}, \varphi_{2} \in \mathcal{L}\left(\Phi_{2}\right)$. Then

$$
\operatorname{Pr}_{\infty}\left(\varphi_{1} \wedge \varphi_{2} \mid K B_{1} \wedge K B_{2}\right)=\operatorname{Pr}_{\infty}\left(\varphi_{1} \mid K B_{1}\right) \times \operatorname{Pr}_{\infty}\left(\varphi_{2} \mid K B_{2}\right)
$$

Proof: See the appendix.

Although very simple, this theorem allows us to deal with such examples as the following:

Example 5.28: Consider the knowledge base $K B_{h e p}$, and a knowledge base stating that $40 \%$ of hospital patients are over 60 years old and that Eric is a patient.

$$
K B_{>60}=_{\text {def }}\|\operatorname{Over60}(x) \mid \operatorname{Patient}(x)\|_{x} \approx_{5} 0.4 \wedge \text { Patient(Eric) }
$$


Then

$$
\begin{aligned}
& \operatorname{Pr}_{\infty}\left(\text { Hep }(\text { Eric }) \wedge \text { Over60 }(\text { Eric }) \mid K B_{\text {hep }} \wedge K B_{>60}\right)= \\
& \quad \operatorname{Pr}_{\infty}\left(\text { Hep }(\text { Eric }) \mid K B_{\text {hep }}\right) \times \operatorname{Pr}_{\infty}\left(\text { Over60 }(\text { Eric }) \mid K B_{>60}\right)=0.8 \times 0.4=0.32
\end{aligned}
$$

In the case of a unary vocabulary (i.e., one containing only unary predicates and constants), Theorem 5.27 can be proved using the connection between the random-worlds method and maximum entropy, which we discuss in Section 6. It is a well-known fact that using maximum entropy often leads to probabilistic independence. The result above proves that, with randomworlds, this phenomenon appears in the non-unary case as well.

We remark that the connection between maximum entropy and independence is sometimes overstated. For example, neither maximum entropy nor random worlds lead to probabilistic independence in examples like the following:

Example 5.29: Consider the knowledge base $K B$, describing a domain of animals:

$$
\|\operatorname{Black}(x) \mid \operatorname{Bird}(x)\|_{x} \approx_{1} 0.2 \wedge\|\operatorname{Bird}(x)\|_{x} \approx_{2} 0.1
$$

It is perfectly consistent for Bird and Black to be probabilistically independent. If this were the case, we would expect the proportion of black animals to be the same as that of black birds. In this case, our degree of belief in Black (Clyde), for some arbitrary animal Clyde, would also be 0.2. However, this is not the case. Since all the predicates here are unary we can use maximum entropy techniques discussed in Section 6 to show that $\operatorname{Pr}_{\infty}($ Black $($ Clyde $) \mid K B)=0.47$. That is, we are almost indifferent about Clyde being black, except for a slight bias due to the fact that he might be a bird and in that case is unlikely to be black.

\subsection{The lottery paradox and unique names}

In Section 3.5 we discussed the lottery paradox and the challenge it poses to theories of default reasoning. How does random-worlds perform?

To describe the original problem in our framework, let Ticket $(x)$ hold if $x$ purchased a lottery ticket. Consider the knowledge base consisting of

$$
K B=\exists ! x \text { Winner }(x) \wedge \forall x(\text { Winner }(x) \Rightarrow \operatorname{Ticket}(x)) .
$$

That is, there is a unique winner, and in order to win one must purchase a lottery ticket. If we also know the size of the lottery, say $N$, we can add to our knowledge base the assertion $\exists^{N} x$ Ticket $(x)$ stating that there are precisely $N$ ticket holders. (This assertion can easily be expressed in first-order logic using equality.) We also assume for simplicity that each individual buys at most one lottery ticket. Then our degree of belief that the individual denoted by a particular constant $c$ wins the lottery is

$$
\operatorname{Pr}_{\infty}\left(\text { Winner }(c) \mid K B \wedge \exists^{N} x \operatorname{Ticket}(x) \wedge \operatorname{Ticket}(c)\right)=\frac{1}{N} .
$$

Our degree of belief that someone wins will obviously be 1 . We would argue that these are reasonable answers. It is true that we do not get the default conclusion that $c$ does not win 
(i.e., degree of belief 0). But since our probabilistic framework can and does express the conclusion that $c$ is very unlikely to win, this is not a serious problem (unlike in systems which either reach a default conclusion or not, with no possibilities in between).

If we do not know the exact number of ticket holders, but have only the qualitative information that this number is "large", then our degree of belief that $c$ wins the lottery is simply $\operatorname{Pr}_{\infty}($ Winner $(c) \mid K B \wedge$ Ticket $(c))=0$, although, as before, $\operatorname{Pr}_{\infty}(\exists x \operatorname{Winner}(x) \mid K B)=1$. In this case we do conclude by default that any particular individual will not win, although we still have degree of belief 1 that someone does win. This shows that the tension Lifschitz sees between concluding a fact for any particular individual and yet not concluding the universal does in fact have a solution in a probabilistic setting such as ours.

Finally, we consider where random worlds fits into Poole's analysis of the lottery paradox. Recall, his argument concentrated on examples in which a class (such as Bird $(x)$ ) is known to be equal to the union of a number of subclasses (Penguin $(x), \operatorname{Emu}(x), \ldots)$, each of which is exceptional in at least one respect. However, using our statistical interpretation of defaults, "exceptional" implies "makes up a negligible fraction of the population". So under our interpretation, Poole's example is inconsistent: we cannot partition the set of birds into a finite number of subclasses, each of which makes up a negligible fraction of the whole set. We view the inconsistency in this case as a feature: it alerts the user that this collection of defaults cannot all be true of the world (given our interpretation of defaults), just as would the inconsistency of the default "Birds typically fly" with "Birds typically do not fly" or "No bird flies".

Our treatment of Poole's example clearly depends on our interpretation of defaults. For instance, we could interpret the default "Birds typically fly" as $\|F l y(x) \mid \operatorname{Bird}(x)\|_{x} \succeq \alpha$ for some appropriately chosen $\alpha$ which is less than 1 . In this case, "exceptional" subclasses (such as penguins which are nonflying birds) can include a nonvanishing fraction of the domain. While allowing an interpretation of default not based on "almost all" does make Poole's $K B$ consistent, it entails giving up many of the attractive properties of the $\approx 1$ representation (such as having default conclusions assigned a degree of belief 1 , and the properties summarized in Theorem 5.3). An alternative solution would be to use the approach presented in [KH92]. Roughly speaking, this approach interprets "almost all" as "arbitrarily close to 1" whenever such an interpretation is consistent (and thus allows us to get the benefits associated with this interpretation). If this interpretation is inconsistent, it takes "almost all" to mean "within $\tau$ of 1 ", for $\tau$ large enough to maintain consistency.

We conclude this section by remarking on another property of the random-worlds method. Applications of default reasoning are often simplified by using the unique names assumption, which says that any two constants should (but perhaps only by default) denote different objects. In random worlds, there is a strong automatic bias towards unique names. If $c_{1}$ and $c_{2}$ are not mentioned anywhere in $K B$, then $\operatorname{Pr}_{\infty}\left(c_{1}=c_{2} \mid K B\right)=0$ (See [GHK94, Lemma D.1] for a formal proof of this fact.) Of course, when we know something about $c_{1}$ and $c_{2}$ it is possible to find examples for which this result fails; for instance $\operatorname{Pr}_{\infty}\left(c_{1}=c_{2} \mid\left(c_{1}=c_{2}\right) \vee\left(c_{2}=c_{3}\right) \vee\left(c_{1}=c_{3}\right)\right)=$ $\frac{1}{3}$. It is hard to give a general theorem saying precisely when the bias towards unique names overrides other considerations. However, we note that both of the "benchmark" examples that Lifschitz has given concerning unique names [Lif89] are correctly handled by random-worlds. For instance, Lifschitz's problem $\mathrm{C} 1$ is:

1. Different names normally denote different people. 
2. The names "Ray" and "Reiter" denote the same person.

3. The names "Drew" and "McDermott" denote the same person.

The desired conclusion here is:

- The names "Ray" and "Drew" denote different people.

Random worlds gives us this conclusion. That is,

$$
\operatorname{Pr}_{\infty}(\text { Ray } \neq \text { Drew } \mid \text { Ray }=\text { Reiter } \wedge \text { Drew }=\text { McDermott })=1 .
$$

Furthermore, we do not have to explicitly state a unique names default.

\section{Random worlds and maximum entropy}

The principle of maximum entropy is a well-known idea, useful for certain types of probabilistic reasoning. Briefly, the entropy of a probability distribution $\mu$ over a finite space $\Omega$ is defined as $H(\mu)=-\sum_{\omega \in \Omega} \mu(\omega) \ln (\mu(\omega))$. It has been argued [SW49] that the entropy measures the amount of "information" in a probability distribution, in the sense of information theory; note that the uniform distribution has the maximum possible entropy. The principle of maximum entropy [Jay78] addresses situations in which we have some constraints on a probability distribution, which may have many solutions, but where we must decide on one particular consistent distribution. The principle asserts that among those distributions satisfying the constraints, the one that should be adopted is the (hopefully unique) distribution having maximum entropy, because it incorporates the least additional information beyond the constraints themselves.

No explicit use of maximum entropy is made by random-worlds. ${ }^{18}$ Indeed, although they are both tools for reasoning about probabilities, the classes of problems considered by the two methods are seemingly disjoint. Nevertheless, it turns out that there is a surprising and very close connection between the random-worlds approach and maximum entropy provided that the language consists only of unary predicates and constants. In this section we briefly describe this connection. This result is of considerable interest simply because it hints at effective computational techniques for random-worlds in the unary case. However, as we discuss below, the connection to random-worlds is interesting for other reasons as well. For instance, we use the connection to show that the maximum-entropy approach to default reasoning, considered in [GMP90], can be embedded in our framework.

To understand the connection to maximum entropy, suppose the language consists of the unary predicate symbols $P_{1}, \ldots, P_{k}$ together with some constant symbols. (Thus, we do not allow either function symbols or higher-arity predicates.) We can consider the $2^{k}$ atoms that can be formed from these predicate symbols, namely, the formulas of the form $Q_{1} \wedge \ldots \wedge Q_{k}$, where each $Q_{i}$ is either $P_{i}$ or $\neg P_{i}$. Then the knowledge base $K B$ can be viewed as simply placing constraints on the proportion of domain elements satisfying each atom. For example,

\footnotetext{
${ }^{18}$ In fact, the postulate of uniform probability over worlds (i.e., indifference) can be seen as a degenerate application of maximum entropy. However, in the context of this paper, this is rather uninteresting and is unrelated to the connection we discuss in the rest of this section.
} 
the formula $\left\|P_{1}(x) \mid P_{2}(x)\right\|_{x}=1 / 2$ says that the proportion of the domain satisfying some atom containing $P_{1}$ as a conjunct is twice the proportion satisfying atoms containing both $P_{1}$ and $P_{2}$ as conjuncts. For unary languages (only) it can be shown that every formula can be rewritten in a canonical form from which constraints on the possible proportions of atoms can be simply derived. Details of this and all other specific results can be found in [GHK94], although the general phenomenon we are about to discuss is addressed in many places, such as [PV89, Sha89] and in statistical physics (e.g., [Lan80]).

The set of constraints generated by $K B$ defines a subset of $[0,1]^{2^{k}}$, which we call $S(K B)$. That is, each vector in $S(K B)$, say $\vec{p}=\left\langle p_{1}, \ldots, p_{2^{k}}\right\rangle$, is a solution of the constraints defined by $K B$ (where $p_{i}$ is the proportion of atom $i$ ). For example, suppose our language contains only the two predicate symbols $\left\{P_{1}, P_{2}\right\}$, so that there are four atoms $A_{1}=P_{1} \wedge P_{2}, A_{2}=P_{1} \wedge \neg P_{2}$, $A_{3}=\neg P_{1} \wedge P_{2}$, and $A_{4}=\neg P_{1} \wedge \neg P_{2}$. Let $K B=\forall x P_{1}(x) \wedge\left\|P_{1}(x) \wedge P_{2}(x)\right\|_{x} \preceq_{1} 0.3$. The first conjunct of $K B$ clearly constrains both $p_{3}$ and $p_{4}$ (the proportion of domain elements satisfying atoms $A_{3}$ and $A_{4}$ ) to be 0 . The second conjunct forces $p_{1}$ to be (approximately) at most $1 / 3$. Thus, $S(K B)=\left\{\left\langle p_{1}, \ldots, p_{4}\right\rangle \in[0,1]^{4}: p_{1} \leq 0.3, p_{3}=p_{4}=0, p_{1}+p_{2}=1\right\}$.

The connection between maximum entropy and the random-worlds method is based on the following observations. With every world $W$, we can associate the vector $\vec{p}^{W}$, where $p_{i}^{W}$ is $\left\|A_{i}(x)\right\|_{x}$ in $W$. Each vector $\vec{p}$ can be viewed as a probability distribution over the space of atoms $A_{1}, \ldots, A_{2^{k}}$; we can therefore associate an entropy with each such vector. We define the entropy of $W$ to be the entropy of $\vec{p}^{W}$. Now, consider some point $\vec{p} \in S(K B)$. What is the number of worlds $W \in \mathcal{W}_{N}$ such that $\vec{p}^{W}=\vec{p}$ ? Clearly, for those $\vec{p}$ where some $p_{i}$ is not an integer multiple of $1 / N$, the answer is 0 . However, for those $\vec{p}$ which are "possible", this number grows asymptotically as $e^{N H(\vec{p})}$. Hence, there are vastly more worlds $W$ for which $\vec{p}^{W}$ is "near" the maximum entropy point of $S(K B)$ than there are worlds elsewhere. This allows us to prove the following result: If, for all sufficiently small $\vec{\tau}$, a formula $\theta$ is true in all worlds around the maximum entropy point(s) of $S(K B)$, then $\operatorname{Pr}_{\infty}(\theta \mid K B)=1$.

In the above example, the maximum-entropy point in $S(K B)$ is $\vec{p}^{*}=(0.3,0.7,0,0)$. Our knowledge base only tells us that the size of atom $A_{1}$ is (approximately) less than or equal to $1 / 3$. But now, consider some small fixed $\epsilon$ and the formula $\theta[\epsilon]=\left\|P_{2}(x)\right\|_{x} \in[0.3-\epsilon, 0.3+\epsilon]$. Since this formula certainly holds at all worlds $W$ where $\vec{p}^{W}$ is sufficiently close to $\vec{p}^{*}$, we conclude that $\operatorname{Pr}_{\infty}(\theta[\epsilon] \mid K B)=1$. This allows us to use Proposition 5.2 to conclude that, for any formula $\varphi, \operatorname{Pr}_{\infty}(\varphi \mid K B)=\operatorname{Pr}_{\infty}(\varphi \mid K B \wedge \theta[\epsilon])$. In particular, this holds for $\varphi=P_{2}(c)$. But now, we can use direct inference to conclude that $\operatorname{Pr}_{\infty}\left(P_{2}(c) \mid K B\right) \in[0.3-\epsilon, 0.3+\epsilon]$. Since this holds for all sufficiently small $\epsilon$, we conclude that $\operatorname{Pr}_{\infty}\left(P_{2}(c) \mid K B\right)=0.3$, as desired. In [GHK94] we formalize this argument and generalize it to more complex examples. These techniques allow us to use a maximum entropy computation as a basis for computing degrees of belief. The resulting procedure applies to many cases not covered by our results in Section 5. Furthermore, since we can take advantage of existing algorithms for computing maximum entropy (see [Gol87] and the references therein), we obtain a technique of potential practical significance.

The connection to maximum entropy is important for many reasons, aside from its computational implications. Maximum entropy has been a popular technique for probabilistic reasoning in AI and elsewhere. Two highly relevant works are the application to inheritance hierarchies by Shastri [Sha89] and to default reasoning by Goldszmidt, Morris, and Pearl [GMP90]. It is desirable that such a popular technique be well-understood and motivated, rather than be 
seen as an ad hoc heuristic. Random worlds, resting on the basic principle of indifference, provides motivation which some may find more convincing than the usual information-theoretic justifications.

Not only does the random-worlds method provide motivation for maximum entropy, it can be viewed as a generalization of it. As discussed above, there is a strong connection between the random-worlds approach and maximum entropy in the unary case (see also [GHK94]). In fact, restricted versions of some of our results from Section 5 can be proved using maximum entropy (see [Sha89]). But our combinatorial proof techniques are more general (and, in fact, simpler) than the ones based directly on entropy. The limitations of maximum entropy are perhaps inescapable, because (as we discuss in detail in [GHK94]) it is reasonable to conjecture that maximum entropy is inherently inapplicable once we move beyond unary predicates.

Finally, our results connecting random worlds to maximum entropy can also be put to use to help clarify the connection between random worlds and previous approaches to applying probabilistic semantics to default reasoning. The mainstay of most of this previous work has been the formalism of $\epsilon$-semantics [GP90]. We briefly review $\epsilon$-semantics here.

Consider a language consisting of propositional formulas (over some finite set of propositional variables $P_{1}, \ldots, P_{k}$ ) and default rules of the form $B \rightarrow C$ (read " $B$ 's are typically $C$ 's"), where $B$ and $C$ are propositional formulas. Let $\Omega$ be the set of $2^{k}$ propositional worlds, corresponding to the possible truth assignments to the variables. Given a probability distribution $\mu$ on $\Omega$, we define $\mu(B)$ to be the probability of the set of worlds where $B$ is true. We say that a distribution $\mu \epsilon$-satisfies a default rule $B \rightarrow C$ if $\mu(C \mid B) \geq 1-\epsilon$.

A parameterized probability distribution (PPD) is a collection $\left\{\mu_{\epsilon}\right\}_{\epsilon>0}$ of probability distributions over $\Omega$, parameterized by $\epsilon$. A PPD $\left\{\mu_{\epsilon}\right\}_{\epsilon>0} \epsilon$-satisfies a set $\mathcal{R}$ of default rules if for every $\epsilon, \mu_{\epsilon} \epsilon$-satisfies every rule $r \in \mathcal{R}$. A set $\mathcal{R}$ of default rules $\epsilon$-entails $B \rightarrow C$ if for every PPD that $\epsilon$-satisfies $\mathcal{R}, \lim _{\epsilon \rightarrow 0} \mu_{\epsilon}(C \mid B)=1$.

As shown in [GP90], $\epsilon$-entailment possesses a number of reasonable properties typically associated with default reasoning, including a preference for more specific information. However, $\epsilon$-entailment is very weak. In particular, as shown by Adams [Ada75], the consequence relation defined by $\epsilon$-entailment satisfies only the five basic properties of default inference given in Section 3.2. Hence, among other limitations, it has no ability to ignore irrelevant information, so it cannot perform any inheritance reasoning.

In order to obtain additional desirable properties, it is necessary to restrict the class of admissible PPD's. Goldszmidt, Morris, and Pearl [GMP90] focus attention on a single PPD: the maximum entropy PPD $\left\{\mu_{\epsilon, \mathcal{R}}^{*}\right\}_{\epsilon>0}$ (See [GMP90] for precise definitions and technical details.) A rule $B \rightarrow C$ is defined to be an $M E$-plausible consequence of $\mathcal{R}$ if $\lim _{\epsilon \rightarrow 0} \mu_{\epsilon, \mathcal{R}}^{*}(C \mid B)=1$. The notion of ME-plausible consequence is analyzed in detail in [GMP90], where it is shown to inherit all the nice properties of $\epsilon$-entailment while having some ability to ignoring irrelevant information. Equally importantly, algorithms are provided for computing the ME-plausible consequences of a set of rules in certain cases (see also [GMP93]).

Our results relating random worlds to maximum entropy can be used to show that the approach of [GMP90] can be embedded in our framework in a straightforward manner. We simply convert all default rules $r$ of the form $B \rightarrow C$ into formulas of the form $\theta_{r}={ }_{\text {def }}$ $\left\|\psi_{C}(x) \mid \psi_{B}(x)\right\|_{x} \approx_{1} 1$, where $\psi_{B}$ is the formula obtained by replacing each occurrence of the propositional variable $p_{i}$ in $B$ with $P_{i}(x)$. Note that the formulas that arise under this con- 
version all use the same approximate equality relation $\approx_{1}$, since the approach of [GMP90] uses the same $\epsilon$ for all default rules. Note also that propositional variables become unary predicates. Hence, default rules become statistical assertions about classes of individuals. Under this translation, we obtain the following theorem (which is proved, and discussed in more detail, in [GHK94]).

Theorem 6.1: Let $c$ be a constant symbol. Using the translation described above, for any set $\mathcal{R}$ of defeasible rules, $B \rightarrow C$ is an $M E$-plausible consequence of $\mathcal{R}$ iff

$$
\operatorname{Pr}_{\infty}\left(\psi_{C}(c) \mid \bigwedge_{r \in \mathcal{R}} \theta_{r} \wedge \psi_{B}(c)\right)=1
$$

Hence, all the computational techniques and results described in [GMP90] carry over to this special case of our approach. Furthermore, unary versions of all of our theorems carry over to the ME-plausible consequence relation. Examples demonstrating inheritance were given in [GMP90], but we can now use Theorem 5.16 to provide a formal characterization of some of the inheritance properties of this consequence relation. It should also be noted that our translation converts the default rules into statistical assertions about classes of individuals and it converts the context, i.e., $B$, into information about a particular individual (whose name we have arbitrarily chosen to be $c$ ). This is in keeping with the intuitive interpretations of rules and context used by propositional default systems (see Section 3.4).

We stress that the assumption that we use the same approximate equality relation is crucial in Theorem 6.1. Geffner [GP92] gives an example of an anomalous conclusion obtained in the system of [GMP90]. Suppose the rule set $\mathcal{R}$ consists of the two rules $P \wedge S \rightarrow Q$ and $R \rightarrow \neg Q$. In this case, the rule $P \wedge S \wedge R \rightarrow Q$ is not an ME-plausible consequence of $\mathcal{R}$. This seems reasonable, as we have evidence for $Q(P \wedge S)$ and against $Q(R)$, and neither piece of evidence is more specific than the other. However, if we add the new rule $P \rightarrow \neg Q$ to $\mathcal{R}$, then $P \wedge S \wedge R \rightarrow Q$ does become an ME-plausible consequence of $\mathcal{R}$. This behavior seems counterintuitive, and is a consequence of [GMP90]'s use of the same $\epsilon$ for all of the rules. Intuitively, what is occurring here is that prior to the addition of the rule $P \rightarrow \neg Q$, the sets $P(x) \wedge S(x)$ and $R(x)$ were of comparable size. The new rule forces $P(x) \wedge S(x)$ to be an $\epsilon$-small subset of $P(x)$, since almost all $P$ 's are $\neg Q$ 's, whereas almost all $P \wedge S$ 's are $Q$ 's. The size of the set $R(x)$, on the other hand, is unaffected. Hence, the default for the $\epsilon$-smaller class $P \wedge S$ now takes precedence over the class $R$. Once we allow a family of approximate equality connectives, each one corresponding to a different $\epsilon$, we are no longer forced to derive this conclusion. An appropriate choice of $\tau_{i}$ can make the default $\|\neg Q(x) \mid R(x)\|_{x} \approx_{i} 1$ so strong that the number of $Q$ 's in the set $R(x)$, and hence the number of $Q$ 's in the subset $P(x) \wedge S(x) \wedge R(x)$, is much smaller than the size of the set $P(x) \wedge S(x) \wedge R(x)$. In this case, the rule $R \rightarrow \neg Q$ takes precedence over the rule $P \wedge S \rightarrow Q$. With no specific information about the relative strengths of the defaults we get non-robustness, as in the Nixon Diamond. That is, we draw no conclusions about $P \wedge S \wedge R \rightarrow Q$.

\section{Problems}

The principle of indifference and maximum entropy have both been subject to criticism. Any such criticism is, at least potentially, relevant to random worlds. Hence, it is important that we 
examine the difficulties that people have found. In this section, we consider problems relating to causal reasoning, language dependence, acceptance, learning, and computation.

\subsection{Causal and temporal information}

The random-worlds method can use knowledge bases which include statistical, first-order, and default information. When is this language sufficient? We suspect that it is, in fact, adequate for most traditional knowledge representation tasks. Nevertheless, the question of adequacy can be subtle. This is certainly the case for the important domain of reasoning about actions, using causal and temporal information. In principle, there would seem to be no difficulty choosing a suitable first-order vocabulary that includes the ability to talk about time explicitly. In the semantics appropriate to many such languages, a world might model an entire temporal sequence of events. However, finding a representation with sufficient expressivity is only part of the problem: we need to know whether the degrees of belief we derive will correctly reflect our intuitions about causal reasoning. It turns out that random worlds gives unintuitive results when used with the most straightforward representations of temporal knowledge.

This observation is not really a new one. As we have observed, the random-worlds method is closely related to maximum entropy (in the context of a unary knowledge base). One significant criticism of maximum entropy techniques has been that they seem to have difficulty dealing with causal information [Hun89, Pea88]. Hence, it is not surprising that the random-worlds method also gives peculiar answers if we represent causal and temporal information naively. On the other hand, Hunter [Hun89] has shown that maximum entropy methods can deal with causal information, provided it is represented appropriately. We have recently shown that by using an appropriate representation (related to Hunter's, but nevertheless distinct), the random-worlds method can also deal well with causal information [BGHK94a]. Indeed, our representation allows us to (a) deal with prediction and explanation problems, (b) represent causal information of the type implicit in Bayesian causal networks [Pea88], and (c) provide a clean and concise solution to the frame problem in the situation calculus [MH69]. In particular, our proposal deals well with some of the standard problems in the area, such as the Yale Shooting Problem [HM87].

The details of the proposal are beyond the scope of this paper. However, the fact we want to emphasize here is that there may be more than one reasonable way to represent our knowledge of a given domain. When one formulation does not work as we expect, we can look for other ways of representing the problem. It will often turn out that the new representation captures some subtle aspects of the domain, that were ignored by the naive representation. (We believe that this is the case with our alternative formulation of reasoning about actions.) We return to this issue a number of times below.

\subsection{Representation dependence}

As we saw above, random worlds suffers from a problem of representation dependence: causal information is treated correctly only if it is represented appropriately. This shows that choosing the "right" representation of our knowledge is important in the context of the random-worlds approach. 
In some ways, this representation dependence is a serious problem because, in practice, how can we know whether we have chosen a good representation or not? Before addressing this, we note that the situation with random worlds is actually not as bad as it might be. As we pointed out in Section 5.1, the random-worlds approach is not sensitive to merely syntactic changes in the knowledge base: logically equivalent knowledge bases always result in the same degrees of belief. So if a changed representation gives different answers, it can only be because we have changed the semantics: we might be using a different ontology, or the new representation might model the world with a different level of detail and accuracy. The representation dependence exhibited by random worlds concerns more than mere syntax. This gives us some hope that the phenomenon can be understood and, at least in some cases, be seen to be entirely appropriate.

Unfortunately, it does seem as if random worlds really is too sensitive; minor and seemingly irrelevant changes can affect things. Perhaps the most disturbing examples concern language dependence, or sensitivity to definitional changes. For instance, suppose the only predicate in our language is White and we take $K B$ to be true. Then $\operatorname{Pr}_{\infty}($ White $(c) \mid K B)=1 / 2$. On the other hand, if we refine $\neg$ White by adding Red and Blue to our language and having $K B^{\prime}$ assert that $\neg$ White is their disjoint union, then $\operatorname{Pr}_{\infty}\left(\right.$ White $\left.(c) \mid K B^{\prime}\right)=1 / 3$. The fact that simply expanding the language and giving a definition of an old notion ( $\neg$ White) in terms of the new notions (Red and Blue) can affect the degree of belief seems to be a serious problem. There are several approaches to dealing with this issue.

One approach to dealing with representation independence is to search for a method of computing degrees of belief that does not suffer from it. To do this, it is important to have a formal definition of representation independence. Once we have such a definition, we can investigate whether there are nontrivial approaches to generating degrees of belief that are representation independent. For example, one might think that the problem lies with our use of point-valued degrees of belief. After all, it is fairly obvious that (under a few very weak assumptions) any approach that gives point-valued degrees of belief that act like probabilities cannot be representation independent. In fact, there has been considerable debate about the "excess precision" forced by point-valued probabilities. Perhaps if we generalize our concept of "degree of belief", say to intervals rather than point values in $[0,1]$, we can address these concerns, as well as avoid the problem of representation dependence. Unfortunately, while interval-valued degrees of belief might well be representation independent in many more circumstances than random worlds, they do not solve the problem. Halpern and Koller, in [HK95], provide a definition of representation independence in the context of probabilistic reasoning, and show that essentially any interesting non-deductive inference procedure cannot be representation independent in their sense.

Another response is to accept this, but to declare that representation dependence is justified, i.e., that the choice of an appropriate vocabulary is indeed a significant one, which does encode some of the information at our disposal. In our example above, we can view the choice of vocabulary as reflecting the bias of the reasoner with respect to the partition of the world into colors. Researchers in machine learning and the philosophy of induction have long realized that bias is an inevitable component of effective inductive reasoning. So we should not be completely surprised if it turns out that the related problem of finding degrees of belief should also depend on the bias. Of course, if this is the case we would hope to have a good intuitive understanding of how the degrees of belief depend on the bias. In particular, we would like to 
give the knowledge base designer some guidelines to selecting the "appropriate" representation. This is an important and seemingly difficult problem in the context of random worlds.

A third response to the problem is to prove representation independence with respect to a large class of queries (see also [HK95]). To understand this approach, consider another example. Suppose that we know that only about half of birds can fly, Tweety is a bird, and Opus is some other individual (who may or may not be a bird). One obvious way to represent this information is to have a language with predicates Bird and Fly, and take the $K B$ to consist of the statements $\|F l y(x) \mid \operatorname{Bird}(x)\|_{x} \approx 0.5$ and $\operatorname{Bird}($ Tweety). It is easy to see that $\operatorname{Pr}_{\infty}($ Fly $($ Tweety $) \mid K B)=0.5$ and $\operatorname{Pr}_{\infty}(\operatorname{Bird}($ Opus $) \mid K B)=0.5$. But suppose that we had chosen to use a different language, one that uses the basic predicates Bird and FlyingBird. We would then take $K B^{\prime}$ to consist of the statements $\|$ FlyingBird $(x) \mid \operatorname{Bird}(x) \|_{x} \approx 0.5, \operatorname{Bird}($ Tweety), and $\forall x($ FlyingBird $(x) \Rightarrow \operatorname{Bird}(x))$. We now get $\operatorname{Pr}_{\infty}($ FlyingBird (Tweety $\left.) \mid K B^{\prime}\right)=0.5$ and $\operatorname{Pr}_{\infty}\left(\operatorname{Bird}(\right.$ Opus $\left.) \mid K B^{\prime}\right)=2 / 3$. Note that our degree of belief that Tweety flies is 0.5 in both cases. In fact, one can give an argument that this conclusion is robust against many "reasonable" representation changes. On the other hand, our degree of belief that Opus is a bird differs in the two representations. Arguably, the fact that our degree of belief that Opus is a bird is language dependent is a direct reflection of the fact that our knowledge base does not contain sufficient information to assign it a single "justified" value. This suggests that it would be useful to characterize those queries that are language independent, while recognizing that not all queries will be.

\subsection{Acceptance and learning}

The most fundamental assumption in this paper is that we are given a knowledge base $K B$ and wish to calculate degrees of belief relative this knowledge. We have not considered how one comes to know $K B$ in the first place. That is, when do we accept information as knowledge? We do not have a good answer to this question. This is unfortunate, since it seems plausible that the processes of gaining knowledge and computing degrees of belief should be interrelated. In particular, Kyburg [Kyb88] has argued that perhaps we might accept assertions that are believed sufficiently strongly. For example, suppose we observe a block $b$ that appears to be white. It could be that we are is not entirely sure that the block is indeed white; it might be some other light color. Nevertheless, if our confidence in White $(b)$ exceeds some threshold, we might accept it (and so include it in $K B$ ).

The problem of acceptance in such examples, concerned with what we learn directly from the senses, is well-known in philosophy [Jef68]. But the problem of acceptance we face is even more difficult than usual, because of our statistical language. Under what circumstances is a statement such as $\|F l y(x) \mid \operatorname{Bird}(x)\|_{x} \approx 0.9$ accepted as knowledge? Although we regard this as an objective statement about the world, it is unrealistic to suppose that anyone could examine all the birds in the world and count how many of them fly. In practice, it seems that this statistical statement would appear in $K B$ if someone inspects a (presumably large) sample of birds and about $90 \%$ of the birds in this sample fly. Then a leap is made: the sample is assumed

to be typical, and we then conclude that $90 \%$ of all birds fly. This would be in the spirit of Kyburg's suggestion so long as we believe that, with high confidence, the full population has statistics similar to those of the sample. 
Unfortunately, the random-worlds method by itself does not support this leap, at least not if we represent the sampling in the most obvious way. That is, suppose we represent our sample using a predicate $S$. We could then represent the fact that $90 \%$ of a sample of birds fly as $\|F l y(x) \mid \operatorname{Bird}(x) \wedge S(x)\|_{x} \approx 0.9$. If the $K B$ consists of this fact and $\operatorname{Bird}($ Tweety), we might hope that $\operatorname{Pr}_{\infty}(F l y($ Tweety $) \mid K B)=0.9$, but it is not. In fact, random worlds treats the birds in $S$ and those outside $S$ as two unrelated populations; it maintains the default degree of belief $(1 / 2)$ that a bird not in $S$ will fly. ${ }^{19}$ Intuitively, random worlds is not treating $S$ as a random sample.

Of course, the failure of the obvious approach does not imply that random worlds is incapable of learning statistics. As was the case for causal reasoning, the solution may be to find an appropriate representation. Perhaps we need a representation reflecting the fact that different individuals do not acquire their properties completely independently of each other. If we see that an animal is tall, it may tell us something about its genetic structure and so, by this mechanism, hint at properties of other animals. But clearly this issue is subtle. If we see a giraffe, this tells us much less about the height of animals in general than it does about other giraffes, and a good representation should reflect this.

While we still hope to find ways of doing sampling within random worlds, we can also look for other ways of coping with the problem of learning. One idea is to add statements about degrees of belief to the knowledge base. Thus, if $20 \%$ of animals in a sample are tall, and we believe that it is appropriate to learn this statistic, then we might add a statement such as $\operatorname{Pr}\left(\|\operatorname{Tall}(x) \mid \operatorname{Animal}(x)\|_{x} \approx_{1} 0.2\right) \geq 0.9$ to the $K B$. Although this does not "automate" the sampling procedure, it allows us to use our belief that a sample is likely to be representative, without committing absolutely to this fact. In particular, this representation allows further evidence to convince the agent that a sample is, in fact, biased. Adding degrees of belief would also let us deal with the problem of acceptance, mentioned at the beginning of this subsection. If we believe that block $b$ is white, but are not certain, we could write $\operatorname{Pr}($ White $(b)) \geq 0.9$. We then do not have to fix an arbitrary threshold for acceptance.

Adding degree of belief statements to a knowledge base is a nontrivial step. Up to now, all the assertions we allowed in a knowledge base were either true or false in a given world. This is not the case for a degree of belief statement. Indeed, our semantics for degrees of belief involve looking at sets of possible worlds. Thus, in order to handle such a statement appropriately, we would need to ensure that our probability distribution over the possible worlds satisfies the associated constraint. A number of different approaches to doing this are discussed in [BGHK94b], and shown to be essentially equivalent.

Yet another approach for dealing with the learning problem is to use a variant of random worlds presented in [BGHK92] called the random-propensities approach. Random worlds has a strong bias towards believing that exactly half the domain has any given property, and this is not always reasonable. Why should it be more likely that half of all birds fly than that a third of them do? Roughly speaking, the random-propensities approach postulates the existence of a parameter denoting the "propensity" of a bird to fly. Initially, all propensities are equally likely. Observing a flying bird gives us information about the propensity of birds to fly, and hence about

\footnotetext{
${ }^{19}$ A related observation, that random worlds cannot do learning (although in a somewhat different sense), was made by Carnap [Car52], who apparently lost his enthusiasm for (his version of) random worlds for precisely this reason.
} 
the flying ability of other birds. As shown in [BGHK92], the random propensities method does, indeed, learn from samples. We can also show [KH96] that the random propensities approach has many of the same attractive properties that we have shown for random worlds, in particular direct inference, specificity, and irrelevance. Unfortunately, random propensities has its own problems. In particular, it learns "too often", i.e., even from arbitrary subsets that are not representative samples. Given the assertion "All giraffes are tall", random propensities would conclude that almost everything is tall. Addressing this problem appropriately is an important issue that deserves further investigation.

\subsection{Computational issues}

Our goal in this research has been to understand some of the fundamental issues involved in first-order probabilistic and default reasoning. Until such issues are understood, it is perhaps reasonable to ignore or downplay concerns about computation. If an ideal normative theory turns out to be impractical for computational reasons, we can still use it as guidance in a search for approximations and heuristics.

As we show in [GHK96b], computing degrees of belief according to random worlds is, indeed, intractable in general. This is not surprising: our language extends first-order logic, for which validity is undecidable. ${ }^{20}$ Although unfortunate, we do not view this as an insurmountable problem. Note that, in spite of its undecideability, first-order logic is nevertheless viewed as a powerful and useful tool. We believe that the situation with random worlds is analogous. Random worlds is not just a computational tool; it is inherently interesting because of what it can tell us about probabilistic reasoning.

But even in terms of computation, the situation with random worlds is not as bleak as it might seem. We have presented one class of much more tractable knowledge bases: those using only unary predicates and constants. We showed in [GHK94] and in Section 6 that, in this case, we can often use maximum entropy as a computational tool in deriving degrees of belief. While computing maximum entropy is also hard in general, there are heuristic techniques that work efficiently in practical cases. As we have already claimed, this class of problems is an important one. In general, many properties of interest can be expressed using unary predicates, since they express properties of individuals. For example, in physics applications we are interested in such predicates as quantum state (see [DD85]). Similarly, AI applications and expert systems typically use only unary predicates ([Che83]) such as symptoms and diseases. In fact, a good case can be made that statisticians tend to reformulate all problems in terms of unary predicates, since an event in a sample space can be identified with a unary predicate [Sha93]. Indeed, most cases where statistics are used, we have a basic unit in mind (an individual, a family, a household, etc.), and the properties (predicates) we consider are typically relative to a single unit (i.e., unary predicates). Thus, results concerning computing degrees of belief for unary knowledge bases are quite significant in practice.

Even for non-unary knowledge bases, there is hope. The intractability proofs given in [GHK96b] use knowledge bases that force the possible worlds to mimic a Turing machine computation. Typical knowledge bases do not usually encode Turing machines! There may therefore

${ }^{20}$ Although, in fact, finding degrees of belief using random worlds is even more intractable than the problem of deciding validity in first-order logic. 
be many cases in which computation is practical. In particular, specific domains typically impose additional structure, which may simplify computation. This seems quite possibly to be the case, in particular, in certain problems that involve reasoning about action.

Furthermore, as we have seen, we can compute degrees of belief in many interesting cases. In particular, we have presented a number of theorems that tell us what the degrees of belief are for certain important classes of knowledge bases and queries. Most of these theorems hold for our language in its full generality, including non-unary predicates. We believe that many more such results could be found. Particularly interesting would be more "irrelevance" results that tell us when large parts of the knowledge base can be ignored. Such results could then be used to reduce apparently complex problems to simpler forms, to which other techniques apply. We have already seen in some of the examples that combining different results can often let us compute degrees of belief in cases where no single result suffices.

Nevertheless, there are many natural knowledge bases that fail to meet the syntactic restrictions required by the theorems we have provided. In particular, the default-reasoning literature includes many quite complicated examples, and we have often found that we cannot understand random-worlds' behavior on these without some (often nontrivial) special-purpose arguments. (Interestingly, it seems to us that the complexity of the required arguments is correlated with how controversial the example is!)

Of course, these difficulties suggest a research strategy: that of characterizing the behavior of the random-worlds method on ever-larger classes of examples. We close by hinting at one of the interesting technical challenges that confronts such a research agenda. It turns out (perhaps not that surprisingly) that a major obstacle is simply the richness of our language. Consider Theorem 5.16. Recall that we had to impose rather severe syntactic restrictions, whose purpose was to ensure that we could identify all the subformulas relevant to a property $\varphi(x)$. The conditions were made so strong because in general it is easy, in a language with as much expressive power as ours, to construct examples in which one part of the knowledge base places nontrivial logical or probabilistic constraints on a superficially (i.e., syntactically) unrelated concept. We certainly believe that random worlds can do inheritance and irrelevance reasoning in a much more comprehensive fashion than suggested by this particular result. But it appears to be hard to state clean, checkable, conditions in a way that does not admit contrived counterexamples. Some progress in this regard is made in [KH96], where additional tools are provided for testing whether formulas can be treated as being irrelevant. In fact, the results of [KH96] can be viewed as steps towards characterizing the properties of a prior distribution that lead to results such as Theorems 5.6 and 5.16, and show that such results apply to priors other than the uniform prior used in the random-worlds method.

\section{Summary}

The random-worlds approach for probabilistic reasoning is derived from two very intuitive ideas: possible worlds and the principle of indifference. In spite of its simple semantics, it has many attractive features:

- It can deal with very rich knowledge bases that involve quantitative information in the form of statistics, qualitative information in the form of defaults, and first-order informa- 
tion. The language is sufficiently powerful for even fairly esoteric demands such as the representation of nested defaults.

- It uses a simple and well-motivated statistical interpretation for defaults. The corresponding semantics allow us to examine the reasonableness of a default with respect to our entire knowledge base, including other default rules.

- It validates many desirable properties, like a preference for more specific information, the ability to ignore irrelevant information, a default assumption of unique names, the ability to combine different pieces of evidence, and more. Most importantly, these properties arise naturally from the very simple semantics of random worlds. In particular, ad hoc assumptions, designed to realize these properties, play no part in the definition of the method.

- It avoids many of the problems that have plagued systems of reference-class reasoning (such as the disjunctive reference class problem) and many of the problems that have plagued systems of non-monotonic reasoning (such as exceptional-subclass inheritance and the lottery paradox). Many systems have been forced to work hard to avoid problems which, in fact, never even arise for random worlds.

- The random-worlds approach subsumes several important reasoning systems, and generalizes them to the case of first-order logic. In particular, it encompasses deductive reasoning, probabilistic reasoning, certain theories of nonmonotonic inference, the principle of maximum entropy, some rules of evidence combination, and more. But it is far more powerful than any of these individual systems.

As we saw in Section 7, there are certainly some problems with the random-worlds method. We believe that these problems are far from insuperable. But, even conceding these problems for the moment, the substantial success of random-worlds supports a few general conclusions.

One conclusion concerns the role of statistics and degrees of belief. The difference between these, and the problem of relating the two, is at the heart of our work. People have long realized that degrees of belief provide a powerful model for understanding rational behavior (for instance, through decision theory). The random-worlds approach shows that it is possible to assign degrees of belief, using a principled technique, in almost any circumstance. The ideal situation, in which we have complete statistical knowledge concerning a domain, is, of course, dealt with appropriately by random worlds. But more realistically, even partial statistical information (which need not be precise) can still be utilized by random worlds to give useful answers. Likewise, completely non-numeric data, which may include defaults and/or a rich first-order theory of some application domain, can be used. Probabilistic reasoning need not make unrealistic demands of the user's knowledge base. Indeed, in a sense it makes less demands that any other reasoning paradigm we know of.

This leads to our next, more general conclusion, which is that many seemingly disparate forms of representation and reasoning can (and, we believe, should) be unified. The first two points listed above suggest that we can take a large step towards this goal by simply finding a powerful language (with clear semantics) that subsumes specialized representations. The advantages we have found (such as a clear and general way of using nested defaults, or 
combining defaults and statistics) apply even if one rejects the random-worlds reasoning method itself. But the language is only part of the answer. Can diverse types of reasoning really be seen as aspects of a single more general system? Clearly this is not always possible; for instance, there are surely some interpretations of "defaults" which have no interesting connection to statistics whatsoever. However, we think that our work demonstrates that the alleged gap between probabilistic reasoning and default reasoning is much narrower than is often thought. In fact, the success of random worlds encourages us to hope that a synthesis between different knowledge representation paradigms is possible in most of the interesting domains.

\section{A Proofs of results}

Theorem 5.5: Assume that $K B \sim_{r w} \varphi$ and $K B \not \psi_{r w} \neg \theta$. Then $K B \wedge \theta \sim_{r w} \varphi$ provided that $\operatorname{Pr}_{\infty}(\varphi \mid K B \wedge \theta)$ exists. Moreover, a sufficient condition for $\operatorname{Pr}_{\infty}(\varphi \mid K B \wedge \theta)$ to exist is that $\operatorname{Pr}_{\infty}(\theta \mid K B)$ exists.

Proof: Since $K B \not \psi_{r w} \neg \theta, \operatorname{Pr}_{\infty}(\neg \theta \mid K B) \neq 1$, so that $\operatorname{Pr}_{\infty}(\theta \mid K B) \neq 0$. Therefore, there exists some $\epsilon>0$ for which we can construct a sequence of pairs $N^{i}, \vec{\tau}^{i}$ as follows: $N^{i}$ is an increasing sequence of domain sizes, $\vec{\tau}^{i}$ is a decreasing sequence of tolerance vectors, and $\operatorname{Pr}_{N^{i}}^{\vec{\tau}^{i}}(\theta \mid K B)>\epsilon$. For these pairs $N^{i}, \vec{\tau}^{i}$ we can conclude using standard probabilistic reasoning that

$$
\operatorname{Pr}_{N^{i}}^{\vec{\tau}^{i}}(\neg \varphi \mid K B \wedge \theta)=\frac{\operatorname{Pr}_{N^{i}}^{\vec{\tau}^{i}}(\neg \varphi \wedge \theta \mid K B)}{\operatorname{Pr}_{N^{i}}^{\vec{\tau}^{i}}(\theta \mid K B)} \leq \frac{\operatorname{Pr}_{N^{i}}^{\vec{\tau}^{i}}(\neg \varphi \mid K B)}{\operatorname{Pr}_{N^{i}}^{\vec{\tau}^{i}}(\theta \mid K B)} .
$$

Since $\operatorname{Pr}_{\infty}(\neg \varphi \mid K B)=0$, it follows that $\lim _{i \rightarrow \infty} \operatorname{Pr}_{N^{i}}^{\vec{\tau}^{i}}(\neg \varphi \mid K B)=0$. Moreover, we know that for all $i, \operatorname{Pr}_{N^{i}}^{\vec{\tau}^{i}}(\theta \mid K B)>\epsilon>0$. We can therefore take the limit as $i \rightarrow \infty$, and conclude that $\lim _{i \rightarrow \infty} \operatorname{Pr}_{N^{i}}^{\vec{\tau}^{i}}(\neg \varphi \mid K B \wedge \theta)=0$. Thus, if $\operatorname{Pr}_{\infty}(\varphi \mid K B \wedge \theta)$ exists, it must be 1 .

For the second half of the theorem, suppose that $\operatorname{Pr}_{\infty}(\theta \mid K B)$ exists. Since $K B \not \downarrow_{r w} \neg \theta$, we must have that $\operatorname{Pr}_{\infty}(\theta \mid K B)=p>0$. Therefore, for all $\vec{\tau}$ sufficiently small and all $N$ sufficiently large (where "sufficiently large" may depend on $\vec{\tau}$ ), we can assume that $\operatorname{Pr}_{N}^{\vec{\tau}}(\theta \mid K B)>\epsilon>0$. But now, for any such pair $N, \vec{\tau}$ we can again prove that

$$
\operatorname{Pr}_{N}^{\vec{\tau}}(\neg \varphi \mid K B \wedge \theta) \leq \frac{\operatorname{Pr}_{N}^{\vec{\tau}}(\neg \varphi \mid K B)}{\operatorname{Pr}_{N}^{\vec{\tau}}(\theta \mid K B)}
$$

Taking the limit, we obtain that $\operatorname{Pr}_{\infty}(\neg \varphi \mid K B \wedge \theta)$ must also have a limit that must be 0 . Hence $\operatorname{Pr}_{\infty}(\varphi \mid K B \wedge \theta)=1$, as desired.

Theorem 5.6: Let $K B$ be a knowledge base of the form $\psi(\vec{c}) \wedge K B^{\prime}$, and assume that for all sufficiently small tolerance vectors $\vec{\tau}$,

$$
K B[\vec{\tau}] \models\|\varphi(\vec{x}) \mid \psi(\vec{x})\|_{\vec{x}} \in[\alpha, \beta] .
$$

If no constant in $\vec{c}$ appears in $K B^{\prime}$, in $\varphi(\vec{x})$, or in $\psi(\vec{x})$, then $\operatorname{Pr}_{\infty}(\varphi(\vec{c}) \mid K B) \in[\alpha, \beta]$, provided the degrees of belief exist. ${ }^{21}$

\footnotetext{
${ }^{21}$ The degree of belief may not exist since $\lim _{\vec{\tau} \rightarrow \overrightarrow{0}} \lim \inf _{N \rightarrow \infty} \operatorname{Pr}_{N}^{\vec{\tau}}(\varphi \mid K B)$ may not be equal to $\lim _{\vec{\tau} \rightarrow \overrightarrow{0}} \limsup _{N \rightarrow \infty} \operatorname{Pr}_{N}^{\vec{\tau}}(\varphi \mid K B)$. However, it follows from the proof of the theorem that both these limits lie in the interval $[\alpha, \beta]$. A similar remark holds for many of our later results.
} 
Proof: First, fix any sufficiently small tolerance vector $\vec{\tau}$, and consider a domain size $N$ for which $K B[\vec{\tau}]$ is satisfiable. The proof strategy is to partition the size $N$ worlds that satisfy $K B[\vec{\tau}]$ into disjoint clusters and then prove that, within each cluster, the probability of $\varphi(\vec{c})$ is in the range $[\alpha, \beta]$. From this, we can show that the (unpartitioned) probability is in this range also.

The size $N$ worlds satisfying $K B[\vec{\tau}]$ are partitioned so that two worlds are in the same cluster if and only if they agree on the denotation of all symbols in the vocabulary $\Phi$ except for the constants in $\vec{c}$. Now consider one such cluster, and let $A \subseteq\{1, \ldots, N\}^{k}$ be the denotation of $\psi(\vec{x})$ inside the cluster. That is, if $W$ is a world in the cluster, then

$$
A=\left\{\left(d_{1}, \ldots, d_{k}\right) \in\{1, \ldots, N\}^{k}:\left(W, V\left[x_{i_{1}} / d_{1}, \ldots, x_{i_{k}} / d_{k}\right], \vec{\tau}\right) \models \psi(\vec{x})\right\} .
$$

Note that, since $\psi(\vec{x})$ does not mention any of the constants in $\vec{c}$, and the denotation of everything else is fixed throughout the cluster, the set $A$ is the same in all worlds $W$ of the cluster. Similarly, let $B \subseteq A$ be the denotation of $\varphi(\vec{x}) \wedge \psi(\vec{x})$ in the cluster. Since the worlds in the cluster all satisfy $K B[\vec{\tau}]$, and $K B[\vec{\tau}] \models\|\varphi(\vec{x}) \mid \psi(\vec{x})\|_{\vec{x}} \in[\alpha, \beta]$, we know that $|B| /|A| \in[\alpha, \beta]$. Since none of the constants in $\vec{c}$ are mentioned in $K B$ except for the statement $\psi(\vec{c})$, each $k$ tuple in $A$ is a legal denotation for $\vec{c}$. There is precisely one world in the cluster for each such denotation, and all worlds in the cluster are of this form. Among those worlds, only those corresponding to tuples in $B$ satisfy $\varphi(\vec{c})$. Therefore, the fraction of worlds in the cluster satisfying $\varphi(\vec{c})$ is $|B| /|A| \in[\alpha, \beta]$.

The probability $\operatorname{Pr}_{N}^{\vec{\tau}}(\varphi(\vec{c}) \mid K B)$ is a weighted average of the probabilities within the individual clusters, so it also has to be in the range $[\alpha, \beta]$.

It follows that $\liminf \inf _{N \rightarrow \infty} \operatorname{Pr}_{N}^{\vec{\tau}}(\varphi(\vec{c}) \mid K B)$ and $\limsup _{N \rightarrow \infty} \operatorname{Pr}_{N}^{\vec{\tau}}(\varphi(\vec{c}) \mid K B)$ are also in the range $[\alpha, \beta]$. Since this holds for every sufficiently small $\vec{\tau}$, we conclude that if both limits

$$
\lim _{\vec{\tau} \rightarrow \overrightarrow{0}} \liminf _{N \rightarrow \infty} \operatorname{Pr}_{N}^{\vec{\tau}}(\varphi(\vec{c}) \mid K B) \text { and } \lim _{\vec{\tau} \rightarrow \overrightarrow{0}} \limsup _{N \rightarrow \infty} \operatorname{Pr}_{N}^{\vec{\tau}}(\varphi \mid K B)
$$

exist and are equal, then $\operatorname{Pr}_{\infty}(\varphi(\vec{c}) \mid K B)$ has to be in the range $[\alpha, \beta]$, as desired.

Theorem 5.16: Let $c$ be a constant and let $K B$ be a knowledge base satisfying the following conditions:

(a) $K B \models \psi_{0}(c)$,

(b) for any expression of the form $\|\varphi(x) \mid \psi(x)\|_{x}$ in $K B$, it is the case that either $K B \models$ $\forall x\left(\psi_{0}(x) \Rightarrow \psi(x)\right)$ or that $K B \models \forall x\left(\psi_{0}(x) \Rightarrow \neg \psi(x)\right)$,

(c) the (predicate, function, and constant) symbols in $\varphi(x)$ appear in $K B$ only on the left-hand side of the conditionals in the proportion expressions described in condition (b),

(d) the constant $c$ does not appear in the formula $\varphi(x)$.

Assume that for all sufficiently small tolerance vectors $\vec{\tau}$ :

$$
K B[\vec{\tau}] \models\left\|\varphi(x) \mid \psi_{0}(x)\right\|_{x} \in[\alpha, \beta] .
$$

Then $\operatorname{Pr}_{\infty}(\varphi(c) \mid K B) \in[\alpha, \beta]$, provided the degree of belief exists. 
Proof: This theorem is proved with the same general strategy we used for Theorem 5.6. That is, for each domain size $N$ and tolerance vector $\vec{\tau}$, we partition the worlds of size $N$ satisfying $K B[\vec{\tau}]$ into clusters and prove that, within each cluster, the probability of $\varphi(c)$ is in the interval $[\alpha, \beta]$. As before, this suffices to prove the result. However the clusters are defined quite differently in this theorem.

We define the clusters as maximal sets of worlds satisfying the following three conditions:

1. All worlds in a cluster must agree on the denotation of every vocabulary symbol except possibly those appearing in $\varphi(x)$. Note that, in particular, they agree on the denotation of the constant $c$. They must also agree as to which elements satisfy $\psi_{0}(x)$; let this set be $A_{0}$.

2. The denotation of symbols in $\varphi$ must also be constant, except possibly when a member of $A_{0}$ is involved. More precisely, let $\overline{A_{0}}$ be the set of domain elements $\{1, \ldots, N\}-A_{0}$. Then for any predicate symbol $R$ or function symbol $f$ of arity $r$ appearing in $\varphi(x)$, and for all worlds $W^{\prime}$ and $W$ in the cluster, if $d_{1}, \ldots, d_{r}, d_{r+1} \in \overline{A_{0}}$ then $R\left(d_{1}, \ldots, d_{r}\right)$ holds in $W^{\prime}$ iff it holds in $W$, and $f\left(d_{1}, \ldots, d_{r}\right)=d_{r+1}$ in $W$ iff $f\left(d_{1}, \ldots, d_{r}\right)=d_{r+1}$ in $W^{\prime}$. In particular, this means that for any constant symbol $c^{\prime}$ appearing in $\varphi(x)$, if it denotes $d^{\prime} \in \overline{A_{0}}$ in $W$, then it must denote $d^{\prime}$ in $W^{\prime}$.

3. All worlds in the cluster are isomorphic with respect to the vocabulary symbols in $\varphi$. More precisely, if $W$ and $W^{\prime}$ are two worlds in the cluster, then there exists some permutation $\pi$ of the domain such that for any predicate symbol $R$ appearing in $\varphi(x)$ and any domain elements $d_{1}, \ldots, d_{r} \in\{1, \ldots, N\}, R\left(d_{1}, \ldots, d_{r}\right)$ holds in $W$ iff $R\left(\pi\left(d_{1}\right), \ldots, \pi\left(d_{r}\right)\right)$ holds in $W^{\prime}$, and similarly for function symbols. In particular, for any constant symbol $c^{\prime}$ appearing in $\varphi(x)$, if it denotes $d^{\prime}$ in $W$, then it denotes $\pi\left(d^{\prime}\right)$ in $W^{\prime}$.

It should be clear that clusters so defined are mutually exclusive and exhaustive.

We now want to prove that each cluster is, in a precise sense, symmetric with respect to the elements in $A_{0}$. That is, let $\pi$ be any permutation of the domain which is the identity on any element outside of $A_{0}$ (i.e., for any $d \notin A_{0}, \pi(d)=d$ ). Let $W$ be any world in our cluster, and let $W^{\prime}$ be the world where all the symbols not appearing in $\varphi$ get the same interpretation as they do in $W$, while the interpretation of the symbols appearing in $\varphi$ is obtained from their interpretation in $W$ by applying $\pi$ as described above. We want to prove that $W^{\prime}$ is also in the cluster. Condition (1) is an immediate consequence of the definition of $W^{\prime}$; the restriction on the choice of $\pi$ implies condition (2); condition (3) holds by definition. It remains only to prove that $W^{\prime} \models K B[\vec{\tau}]$. Because of condition (c) in the statement of the theorem, and the fact that vocabulary symbols not in $\varphi$ have the same denotation in $W$ and in $W^{\prime}$, this can fail to happen only if some expression $\|\varphi(x) \mid \psi(x)\|_{x}$ has different values in $W$ and in $W^{\prime}$. We show that this is impossible.

It is easy to see that for all domain elements $d$, we have $(W, V, \vec{\tau}) \models \psi(x)$ iff $\left(W^{\prime}, V, \vec{\tau}\right) \models \psi(x)$ (where $V$ is a valuation mapping $x$ to $d$ ), since the symbols not in $\varphi$ get the same interpretation in both $W$ and $W^{\prime}$. On the other hand, if $\varphi^{\prime}$ is a formula that mentions only the symbols appearing in $\varphi$, then a straightforward induction on the structure of $\varphi^{\prime}$ can be used to show that $(W, V, \vec{\tau}) \models \varphi^{\prime}(x)$ iff $\left(W^{\prime}, \pi \circ V, \vec{\tau}\right) \models \varphi^{\prime}(x)$, where $\pi \circ V$ is the valuation that maps $x$ to $\pi(V(x))$. Thus, if $B$ is the set of elements satisfying $\varphi(x)$ in $W$, then $\pi(B)$ is the set of elements satisfying 
$\varphi(x)$ in $W^{\prime}$. Let $A$ be the set of domain elements satisfying $\psi(x)$ for worlds in this cluster. We want to show that $|B \cap A| /|A|=|\pi(B) \cap A| /|A|$ or, equivalently, that $|B \cap A|=|\pi(B) \cap A|$. By our observations above, the set of domain elements satisfying $\varphi(x) \wedge \psi(x)$ in $W^{\prime}$ is $\pi(B) \cap A$. By condition (b) there are only two cases: Either $K B \models \forall x\left(\psi_{0}(x) \Rightarrow \neg \psi(x)\right)$, in which case $A_{0}$ and $A$ are disjoint, or $K B \models \forall x\left(\psi_{0}(x) \Rightarrow \psi(x)\right)$, so that $A_{0} \subseteq A$. In the first case, since $\pi$ is the identity off $A_{0}$, it is easy to see that $\pi(B) \cap A=B \cap A$, and we are done. In the second case, because $A_{0} \subseteq A, \pi$ is a permutation of $A$ into itself, so we must still have $|\pi(B) \cap A|=|B \cap A|$. We conclude that $W^{\prime}$ does satisfy $K B[\vec{\tau}]$, and is therefore also in the cluster. Since we restricted the cluster to consist only of worlds that are isomorphic to $W$ in the above sense, and we have now proved that all worlds formed in this way are in the cluster, the cluster contains precisely all such worlds.

Having defined the clusters, we want to show that the degree of belief of $\varphi(c)$ is in the range $[\alpha, \beta]$ when we look at any single cluster. By assumption, $K B[\vec{\tau}] \models\left\|\varphi(x) \mid \psi_{0}(x)\right\|_{x} \in[\alpha, \beta]$. Therefore, for each world in the cluster, the subset of the elements of $A_{0}$ that satisfy $\varphi(x)$ is in the interval $[\alpha, \beta]$. Moreover, by condition (a), $K B$ also entails the assertion $\psi_{0}(c)$. Therefore, the denotation of $c$ is some domain element $d$ in $A_{0}$. Condition (d) says that $c$ does not appear in $\varphi$, and so the denotation of $c$ is the same for all worlds in the cluster. Now consider a world $W$ in the cluster, and let $B$ be the subset of $A_{0}$ whose members satisfy $\varphi(x)$ in $W$. We have shown that every permutation of the elements in $A_{0}$ (leaving the remaining elements constant) has a corresponding world in the cluster. In particular, all possible subsets $B^{\prime}$ of size $|B|$ are possible denotations for $\varphi(x)$ in worlds in the cluster. Furthermore, because of symmetry, they are all equally likely. It follows that the fixed element $d$ satisfies $\varphi(x)$ in precisely $|B| /\left|A_{0}\right|$ of the worlds in the cluster. Since $|B| /\left|A_{0}\right| \in[\alpha, \beta]$, the probability of $\varphi(c)$ in any one cluster is in this range also.

As in Theorem 5.6, the truth of this fact for each cluster implies its truth in general and at the limit. In particular, since $K B[\vec{\tau}] \models\left\|\varphi(x) \mid \psi_{0}(x)\right\|_{x} \in[\alpha, \beta]$ for every sufficiently small $\vec{\tau}$, we conclude that $\operatorname{Pr}_{\infty}(\varphi(c) \mid K B) \in[\alpha, \beta]$, if the limit exists.

Theorem 5.23: $\quad$ Suppose KB has the form

$$
\bigwedge_{i=1}^{m}\left(\alpha_{i} \preceq_{\ell_{i}}\left\|\varphi(x) \mid \psi_{i}(x)\right\|_{x} \preceq_{r_{i}} \beta_{i}\right) \wedge \psi_{1}(c) \wedge K B^{\prime}
$$

and, for all $i, K B \models \forall x\left(\psi_{i}(x) \Rightarrow \psi_{i+1}(x)\right) \wedge \neg\left(\left\|\psi_{1}(x)\right\|_{x} \approx_{1} 0\right)$. Assume also that no symbol appearing $\varphi(x)$ appears in $K B^{\prime}$ or in any $\psi_{i}(c)$. Further suppose that, for some $j,\left[\alpha_{j}, \beta_{j}\right]$ is the tightest interval. That is, for all $i \neq j, \alpha_{i}<\alpha_{j}<\beta_{j}<\beta_{i}$. Then, if the degree of belief exists,

$$
\operatorname{Pr}_{\infty}(\varphi(c) \mid K B) \in\left[\alpha_{j}, \beta_{j}\right] .
$$

Proof: The proof of the theorem is based on the following result. Consider any $K B$ of the form

$$
\neg\left(\left\|\psi^{\prime}(x)\right\|_{x} \approx_{1} 0\right) \wedge \forall x\left(\psi^{\prime}(x) \Rightarrow \psi(x)\right) \wedge \alpha \preceq_{\ell}\|\varphi(x) \mid \psi(x)\|_{x} \preceq_{r} \beta \wedge K B^{\prime}
$$

where none of $K B^{\prime}, \psi(x), \psi^{\prime}(x)$ mention any symbol appearing in $\varphi(x)$. Then, for any $\epsilon>0$,

$$
\operatorname{Pr}_{\infty}\left(\alpha-\epsilon \leq\left\|\varphi(x)\left|\psi^{\prime}(x) \|_{x} \leq \beta+\epsilon\right| K B\right)=1 .\right.
$$


Note that this is quite similar in spirit to Theorem 5.16, where we proved that (under certain conditions) an individual $c$ satisfying $\psi(c)$ "inherits" the statistics over $\psi(x)$; that is, the degree of belief is derived from these statistics. Not surprisingly, the proof of the new result is similar to that of Theorem 5.16, and we refer the reader to that proof for many of the details.

We begin by clustering worlds exactly as in the earlier proof, with $\psi(x)$ playing the role of the earlier $\psi_{0}(x)$. Now consider any particular cluster and let $A$ be the corresponding denotation of $\psi(x)$. In the cluster, the proportion of $A$ that satisfies $\varphi(x)$ is some $\gamma$ such that $\alpha-\tau_{\ell} \leq \gamma \leq \beta+\tau_{r}$. (Recall that $\tau_{\ell}$ and $\tau_{r}$ are the tolerances associated with the approximate comparisons $\approx_{\ell}$ and $\approx_{r}$ in $\left.K B\right)$. In this cluster, the denotation of $\varphi(x)$ in $A$ ranges over subsets of $A$ of size $\gamma|A|$. From the proof of Theorem 5.16, we know that there is, in fact, an equal number of worlds in the cluster corresponding to every such subset.

Now let $A^{\prime}$ be the denotation of $\psi^{\prime}(x)$ in the cluster (recall that it follows from the construction of the clusters that all worlds in a cluster have the same denotation for $\psi^{\prime}(x)$ ). For a proportion $\gamma^{\prime} \in[0,1]$, we are interested in computing the fraction of worlds in the cluster such that the proportion of $\varphi(x)$ in $A^{\prime}$ is $\gamma^{\prime}$. From our discussion above, it follows that this is a purely combinatorial question: given a set $A$ of size $n$ and a subset $A^{\prime}$ of size $n^{\prime}$, how many ways are there of choosing $\gamma n$ elements (representing the elements for which $\varphi(x)$ holds) so that $\gamma^{\prime} n^{\prime}$ elements come from $A^{\prime}$ ? We estimate this using the observation that the distribution of $\gamma^{\prime} n^{\prime}$ is derived from a process of sampling without replacement. ${ }^{22}$ Hence, it behaves according to the well-known hypergeometric distribution (see, for example, [LM81]). We can thus conclude that $\gamma^{\prime}$ is distributed with mean $\gamma$ and variance

$$
\frac{\gamma(1-\gamma)\left(n-n^{\prime}\right)}{(n-1) n^{\prime}} \leq \frac{\gamma(1-\gamma)}{n^{\prime}} \leq \frac{1}{4 n^{\prime}}
$$

Since $K B \models \neg\left(\left\|\psi^{\prime}(x)\right\|_{x} \approx_{1} 0\right)$, we know that $n^{\prime}=\left|A^{\prime}\right| \geq \tau_{1} N$. Thus, this variance tends to 0 as $N$ grows large. Now, consider the event: "a world in the cluster has a proportion of $\varphi(x)$ within $A^{\prime}$ which is not in the interval $[\gamma-\epsilon, \gamma+\epsilon]$ ". By Chebychev's inequality, this is bounded from above by some small probability $p_{N}$ which depends only on $\tau_{1} N$. That is, the fraction of worlds in each cluster that have the "wrong" proportion is at most $p_{N}$. Since this is the case for every cluster, it is also true in general. More precisely, the fraction of overall worlds for which $\left\|\varphi(x) \mid \psi^{\prime}(x)\right\|_{x} \notin\left[\alpha-\tau_{\ell}-\epsilon, \beta+\tau_{r}+\epsilon\right]$ is at most $p_{N}$. But this probability goes to 0 as $N$ tends to infinity. Therefore,

$$
\operatorname{Pr}_{\infty}^{\vec{\tau}}\left(\alpha-\tau_{\ell}-\epsilon \leq\left\|\varphi(x)\left|\psi^{\prime}(x) \|_{x} \leq \beta+\tau_{r}+\epsilon\right| K B\right)=1 .\right.
$$

As $\vec{\tau} \rightarrow \overrightarrow{0}$ we can simply omit $\tau_{\ell}$ and $\tau_{r}$, proving the required result.

It is now a simple matter to prove the theorem itself. Consider the following modification $K B^{\prime \prime}$ of the $K B$ given in the statement of the theorem:

$$
\bigwedge_{i=j}^{m}\left(\alpha_{i} \preceq_{\ell_{i}}\left\|\varphi(x) \mid \psi_{i}(x)\right\|_{x} \preceq_{r_{i}} \beta_{i}\right) \wedge \psi_{1}(c) \wedge K B^{\prime},
$$

\footnotetext{
${ }^{22}$ There are, in fact, a number of ways to solve this problem. One alternative is to use an entropy-based technique. We can do this because, at this point in the proof, it no longer matters whether $K B$ uses nonunary predicates or not; we can therefore safely apply techniques that usually only work in the unary case.
} 
where we eliminate the statistics for the reference classes that are contained in $\psi_{j}$ (the more specific reference classes). From Theorem 5.16 we can conclude that $\operatorname{Pr}_{\infty}\left(\varphi(c) \mid K B^{\prime \prime}\right) \in\left[\alpha_{j}, \beta_{j}\right]$ (the conditions of that theorem are clearly satisfied). But we also know, from the result above, that for each $\psi_{i}$, for $i<j$ :

$$
\operatorname{Pr}_{\infty}\left(\alpha_{j}-\epsilon \leq\left\|\varphi(x)\left|\psi^{\prime}(x) \|_{x} \leq \beta_{j}+\epsilon\right| K B^{\prime \prime}\right)=1\right.
$$

For sufficiently small $\epsilon>0$, the assertion that

$$
\alpha_{j}-\epsilon \leq\left\|\varphi(x) \mid \psi^{\prime}(x)\right\|_{x} \leq \beta_{j}+\epsilon
$$

logically implies that

$$
\alpha_{i} \preceq \ell_{i}\left\|\varphi(x) \mid \psi^{\prime}(x)\right\|_{x} \preceq r_{i} \beta_{i},
$$

so that this latter assertion also has probability 1 given $K B^{\prime \prime}$. We therefore also have probability 1 (given $K B^{\prime \prime}$ ) in the finite conjunction

$$
\bigwedge_{i=1}^{j}\left(\alpha_{i} \preceq_{\ell_{i}}\left\|\varphi(x) \mid \psi^{\prime}(x)\right\|_{x} \preceq_{r_{i}} \beta_{i}\right) .
$$

We can now apply Theorem 5.2 to conclude that we can add this finite conjunction to $K B^{\prime \prime}$ without affecting any of the degrees of belief. But the knowledge base resulting from adding this conjunction to $K B^{\prime \prime}$ is precisely the original $K B$. We conclude that

$$
\operatorname{Pr}_{\infty}(\varphi(c) \mid K B)=\operatorname{Pr}_{\infty}\left(\varphi(c) \mid K B^{\prime \prime}\right) \in\left[\alpha_{j}, \beta_{j}\right]
$$

as required.

Theorem 5.26: $\quad$ Let $P$ be a unary predicate, and consider a knowledge base $K B$ of the following form:

$$
\bigwedge_{i=1}^{m}\left(\left\|P(x) \mid \psi_{i}(x)\right\|_{x} \approx_{i} \alpha_{i} \wedge \psi_{i}(c)\right) \wedge \bigwedge_{\substack{i, j=1 \\ i \neq j}}^{m} \exists ! x\left(\psi_{i}(x) \wedge \psi_{j}(x)\right)
$$

where either $\alpha_{i}<1$ for all $i=1, \ldots, m$, or $\alpha_{i}>0$ for all $i=1, \ldots, m$. Then, if neither $P$ nor $c$ appear anywhere in the formulas $\psi_{i}(x)$, then

$$
\operatorname{Pr}_{\infty}(P(c) \mid K B)=\delta\left(\alpha_{1}, \ldots, \alpha_{m}\right)=\frac{\prod_{i=1}^{m} \alpha_{i}}{\prod_{i=1}^{m} \alpha_{i}+\prod_{i=1}^{m}\left(1-\alpha_{i}\right)}
$$

Proof: Assume without loss of generality that $\alpha_{i}>0$ for $i=1, \ldots, m$. As in previous theorems, we prove the result by dividing the worlds into clusters. More precisely, consider any $\vec{\tau}$ such that $\alpha_{i}-\tau_{i}>0$. Let $\beta_{i}=\min \left(\alpha_{i}+\tau_{i}, 1\right)$. For any such $\vec{\tau}$ and any domain size $N$, we divide the worlds of size $N$ satisfying $K B[\vec{\tau}]$ into clusters, and prove that, within each cluster, the probability of $\varphi(c)$ is in the interval $\left[\delta\left(\alpha_{1}-\tau_{1}, \ldots, \alpha_{m}-\tau_{m}\right), \delta\left(\beta_{1}, \ldots, \beta_{m}\right)\right]$. Since $\delta$ is a continuous function at these points, this suffices to prove the theorem.

We partition the worlds satisfying $K B[\vec{\tau}]$ into maximal clusters that satisfy the following three conditions: 
1. All worlds in a cluster must agree on the denotation of every vocabulary symbol except for $P$. In particular, the denotations of $\psi_{1}(x), \ldots, \psi_{m}(x)$ is fixed. For $i=1, \ldots, m$, let $A_{i}$ denote the denotation of $\psi_{i}(x)$ in the cluster, and let $n_{i}$ denote $\left|A_{i}\right|$.

2. All worlds in a cluster must have the same denotation of $P$ for elements in $\bar{A}=\{1, \ldots, N\}-$ $\cup_{i=1}^{m} A_{i}$.

3. For all $i=1, \ldots, m$, all worlds in the cluster must have the same number of elements $r_{i}$ satisfying $P$ within each set $A_{i}$. Note that, since all worlds in the cluster satisfy $K B[\vec{\tau}]$, it follows that $r_{i} / n_{i} \in\left[\alpha_{i}-\tau_{i}, \beta_{i}\right]$ for $i=1, \ldots, m$.

Now, consider a cluster as defined above. The assumptions of the theorem imply that, besides the proportion constraints defined by the numbers $r_{i}$, there are no other constraints on the denotation of $P$ within the sets $A_{1}, \ldots, A_{m}$. Therefore, all possible denotations of $P$ satisfying these constraints are possible. Let $d$ be the denotation of $c$ in this cluster. Our assumptions guarantee that $d$ is the only member of $A_{i} \cap A_{j}$. Hence, the number of elements of $A_{i}$ for which $P$ has not yet been chosen is $n_{i}-1$. In worlds that satisfy $P(c)$, precisely $r_{i}-1$ of these elements must satisfy $P$. Since the $A_{i}$ are disjoint except for $d$, the choice of $P$ within each $A_{i}$ can be made independently of the other choices. Therefore, the number of worlds in the cluster where $P(c)$ holds is

$$
\prod_{i=1}^{m}\left(\begin{array}{c}
n_{i}-1 \\
r_{i}-1
\end{array}\right) .
$$

Similarly, the number of worlds in the cluster for which $P(c)$ does not hold is

$$
\prod_{i=1}^{m}\left(\begin{array}{c}
n_{i}-1 \\
r_{i}
\end{array}\right) .
$$

Therefore, the fraction of worlds in the cluster satisfying $P(c)$ is:

$$
\begin{aligned}
\frac{\prod_{i=1}^{m}\left(\begin{array}{c}
n_{i}-1 \\
r_{i}-1
\end{array}\right)}{\prod_{i=1}^{m}\left(\begin{array}{c}
n_{i}-1 \\
r_{i}-1
\end{array}\right)+\prod_{i=1}^{m}\left(\begin{array}{c}
n_{i}-1 \\
r_{i}
\end{array}\right)} & =\frac{\prod_{i=1}^{m} r_{i}}{\prod_{i=1}^{m} r_{i}+\prod_{i=1}^{m}\left(n_{i}-r_{i}\right)} \\
& =\frac{\prod_{i=1}^{m} r_{i} / n_{i}}{\prod_{i=1}^{m} r_{i} / n_{i}+\prod_{i=1}^{m}\left(n_{i}-r_{i}\right) / n_{i}} \\
& =\delta\left(r_{1} / n_{1}, \ldots, r_{m} / n_{m}\right) .
\end{aligned}
$$

Since $\delta$ is easily seen to be monotonically increasing in each of its arguments and $r_{i} / n_{i} \in$ $\left[\alpha_{i}-\tau_{i}, \beta_{i}\right]$, we must have that $\delta\left(r_{1} / n_{1}, \ldots, r_{m} / n_{m}\right)$ is in the interval $\left[\delta\left(\alpha_{1}-\tau_{1}, \ldots, \alpha_{m}-\right.\right.$ $\left.\left.\tau_{m}\right), \delta\left(\beta_{1}, \ldots, \beta_{m}\right)\right]$. Using the same argument as in the previous theorems and the continuity of $\delta$, we deduce the desired result.

Theorem 5.27: Let $\Phi_{1}$ and $\Phi_{2}$ be two subvocabularies of $\Phi$ that are disjoint except for the constant c. Consider $K B_{1}, \varphi_{1} \in \mathcal{L}\left(\Phi_{1}\right)$ and $K B_{2}, \varphi_{2} \in \mathcal{L}\left(\Phi_{2}\right)$. Then

$$
\operatorname{Pr}_{\infty}\left(\varphi_{1} \wedge \varphi_{2} \mid K B_{1} \wedge K B_{2}\right)=\operatorname{Pr}_{\infty}\left(\varphi_{1} \mid K B_{1}\right) \times \operatorname{Pr}_{\infty}\left(\varphi_{2} \mid K B_{2}\right)
$$

Proof: Fix $N, \vec{\tau}$, and $d$ with $1 \leq d \leq N$. Given a vocabulary $\Psi$ containing $c$, let $\operatorname{worlds}_{N}^{d, \vec{\tau}, \Psi}(\xi)$ consist of all worlds in $\mathcal{W}_{N}(\Psi)$ such that $(W, \vec{\tau}) \models \xi$ and the denotation of $c$ in $W$ is $d$, and 
let \# worlds ${ }_{N}^{d, \vec{\tau}, \Psi}(\xi)=\mid$ worlds $_{N}^{d, \vec{\tau}, \Psi}(\xi) \mid{ }^{23}$ It should be clear that for each choice of $d$, the sets $\operatorname{worlds}_{N}^{d, \vec{\tau}, \Phi}(\xi)$ have equal size. Thus, \# $\operatorname{worlds}_{N}^{\vec{\tau}, \Phi}(\xi)=N \# \operatorname{worlds}_{N}^{d, \vec{\tau}, \Phi}(\xi)$. If $\xi_{1}$ is a formula in $\Phi_{1}$ and $\xi_{2}$ is a formula in $\Phi_{2}$, then there is clearly a bijection between $\operatorname{worlds}_{N}^{d, \vec{\tau}, \Phi_{1} \cup \Phi_{2}}\left(\xi_{1} \wedge \xi_{2}\right)$ and $\operatorname{worlds}_{N}^{d, \vec{\tau}, \Phi_{1}}\left(\xi_{1}\right) \times \operatorname{worlds}_{N}^{d, \vec{\tau}, \Phi_{2}}\left(\xi_{2}\right)$. It follows that \# $\operatorname{worlds}_{N}^{d, \vec{\tau}, \Phi_{1} \cup \Phi_{2}}\left(\xi_{1} \wedge \xi_{2}\right)=\# \operatorname{worlds}_{N}^{d, \vec{\tau}, \Phi_{1}}\left(\xi_{1}\right) \times$ $\# \operatorname{worlds}_{N}^{d, \vec{\tau}, \Phi_{2}}\left(\xi_{2}\right)$. Since \# $\operatorname{worlds}_{N}^{\vec{\tau}, \Phi}(\xi)=N \# \operatorname{worlds}_{N}^{d, \vec{\tau}, \Phi}(\xi)$, we immediately get

$$
\begin{aligned}
& \operatorname{Pr}_{N}^{\vec{\tau}, \Phi_{1} \cup \Phi_{2}}\left(\varphi_{1} \wedge \varphi_{2} \mid K B_{1} \wedge K B_{2}\right) \\
& =\frac{\# \text { worlds }_{N}^{\vec{\tau}, \Phi_{1} \cup \Phi_{2}}\left(\varphi_{1} \wedge K B_{1} \wedge \varphi_{2} \wedge K B_{2}\right)}{\# \text { worlds }_{N}^{\vec{\tau}, \Phi_{1} \cup \Phi_{2}}\left(K B_{1} \wedge K B_{2}\right)} \\
& =\frac{\# \text { worlds }_{N}^{d, \vec{\tau}, \Phi_{1} \cup \Phi_{2}}\left(\varphi_{1} \wedge K B_{1} \wedge \varphi_{2} \wedge K B_{2}\right)}{\# \text { worlds }_{N}^{d, \vec{\tau}, \Phi_{1} \cup \Phi_{2}}\left(K B_{1} \wedge K B_{2}\right)} \\
& =\frac{\# \operatorname{worlds}_{N}^{d, \vec{\tau}, \Phi_{1}}\left(\varphi_{1} \wedge K B_{1}\right) \times \# \text { worlds }_{N}^{d, \vec{\tau}, \Phi_{2}}\left(\varphi_{2} \wedge K B_{2}\right)}{\# \operatorname{worlds}_{N}^{d, \vec{\tau}, \Phi_{1}}\left(K B_{1}\right) \times \# \operatorname{worlds}_{N}^{d, \vec{\tau}, \Phi_{2}}\left(K B_{2}\right)} \\
& =\frac{\# \operatorname{worlds}_{N}^{\vec{\tau}, \Phi_{1}}\left(\varphi_{1} \wedge K B_{1}\right) \times \# \text { worlds }_{N}^{\vec{\tau}, \Phi_{2}}\left(\varphi_{2} \wedge K B_{2}\right)}{\# \operatorname{worlds}_{N}^{\vec{\tau}, \Phi_{1}}\left(K B_{1}\right) \times \# \operatorname{worlds}_{N}^{\vec{\tau}, \Phi_{2}}\left(K B_{2}\right)} \\
& =\operatorname{Pr}_{N}^{\vec{\tau}, \Phi_{1}}\left(\varphi_{1} \mid K B_{1}\right) \times \operatorname{Pr}_{N}^{\vec{\tau}, \Phi_{2}}\left(\varphi_{2} \mid K B_{2}\right) \text {. }
\end{aligned}
$$

Taking limits, we get that $\operatorname{Pr}_{\infty}^{\Phi_{1} \cup \Phi_{2}}\left(\varphi_{1} \wedge \varphi_{2} \mid K B_{1} \wedge K B_{2}\right)=\operatorname{Pr}_{\infty}^{\Phi_{1}}\left(\varphi_{1} \mid K B_{1}\right) \cdot \operatorname{Pr}_{\infty}^{\Phi_{2}}\left(\varphi_{2} \mid K B_{2}\right)$. As observed in [GHK96b], for all formulas $\varphi$ and $K B$, if $\Phi \supseteq \Phi^{\prime}$, then $\operatorname{Pr}_{\infty}^{\Phi}(\varphi \mid K B)=\operatorname{Pr}_{\infty}^{\Phi^{\prime}}(\varphi \mid K B)$. (Intuitively, this is because the effect of changing the vocabulary cancels out in the numerator and denominator.) We thus get $\operatorname{Pr}_{\infty}\left(\varphi_{1} \wedge \varphi_{2} \mid K B_{1} \wedge K B_{2}\right)=\operatorname{Pr}_{\infty}\left(\varphi_{1} \mid K B_{1}\right) \cdot \operatorname{Pr}_{\infty}\left(\varphi_{2} \mid K B_{2}\right)$, as desired.

\section{References}

[Ada75] E. Adams. The Logic of Conditionals. D. Reidel, Dordrecht, Netherlands, 1975.

[Ash93] N. Asher. Extensions for commonsense entailment. In Proceedings of the IJCAI Workshop on Conditionals in Knowledge Representation, pages 26-41, 1993.

[Bac90] F. Bacchus. Representing and Reasoning with Probabilistic Knowledge. MIT Press, Cambridge, Mass., 1990.

$\left[\mathrm{BCD}^{+} 93\right] \quad$ S. Benferhat, C. Cayrol, D. Dubois, J. Lang, and H. Prade. Inconsistency management and prioritized syntax-based entailment. In Proc. Thirteenth International Joint Conference on Artificial Intelligence (IJCAI '93), pages 640-645, 1993.

[BGHK92] F. Bacchus, A. J. Grove, J. Y. Halpern, and D. Koller. From statistics to belief. In Proc. Tenth National Conference on Artificial Intelligence (AAAI '92), pages 602-608. 1992.

\footnotetext{
${ }^{23}$ Note that we are careful to mention the vocabulary in the superscript here, rather than suppressing it as we have up to now. This is because the vocabulary used plays a significant role in this proof.
} 
[BGHK93] F. Bacchus, A. J. Grove, J. Y. Halpern, and D. Koller. Statistical foundations for default reasoning. In Proc. Thirteenth International Joint Conference on Artificial Intelligence (IJCAI '93), pages 563-569, 1993. Available by anonymous ftp from logos.uwaterloo.ca/pub/bacchus or via WWW at http://logos.uwaterloo.ca.

[BGHK94a] F. Bacchus, A. J. Grove, J. Y. Halpern, and D. Koller. Forming beliefs about a changing world. In Proc. Twelfth National Conference on Artificial Intelligence (AAAI '94), pages 222-229, 1994. Available by anonymous ftp from logos.uwaterloo.ca/pub/bacchus or via WWW at http://logos.uwaterloo.ca.

[BGHK94b] F. Bacchus, A. J. Grove, J. Y. Halpern, and D. Koller. Generating new beliefs from old. pages 37-45, 1994. Available by anonymous ftp from logos.uwaterloo.ca/pub/bacchus or via WWW at http://logos.uwaterloo.ca.

[BGHK94c] F. Bacchus, A. J. Grove, J. Y. Halpern, and D. Koller. A response to: "Believing on the basis of evidence". Computational Intelligence, 10(1):21-25, 1994.

[Bou91] C. Boutilier. Conditional Logics for Default Reasoning and Belief Revision. PhD thesis, Department of Computer Science, University of Toronto, 1991.

[Car50] R. Carnap. Logical Foundations of Probability. University of Chicago Press, Chicago, 1950.

[Car52] R. Carnap. The Continuum of Inductive Methods. University of Chicago Press, Chicago, 1952.

[Che83] P. C. Cheeseman. A method of computing generalized Bayesian probability values for expert systems. In Proc. Eighth International Joint Conference on Artificial Intelligence (IJCAI '83), pages 198-202. 1983.

[Chu91] R. Chuaqui. Truth, possibility, and probability: new logical foundations of probability and statistical inference. North-Holland, Amsterdam, 1991.

[DD85] K. G. Denbigh and J. S. Denbigh. Entropy in Relation to Incomplete Knowledge. Cambridge University Press, Cambridge, U.K., 1985.

[Del88] J. P. Delgrande. An approach to default reasoning based on a first-order conditional logic: Revised report. Artificial Intelligence, 36:63-90, 1988.

[EKP91] D. W. Etherington, S. Kraus, and D. Perlis. Nonmonotonicity and the scope of reasoning. Artificial Intelligence, 2:221-261, 1991.

[Gab84] D. Gabbay. Theoretical foundations for nonmonotonic reasoning in expert systems. In K. R. Apt, editor, Proceedings of the NATO Advanced Study Institute on logics and models of concurrent systems. Springer-Verlag, 1984.

[Gef92] H. Geffner. Default Reasoning: Causal and Conditional Theories. MIT Press, Cambridge, Mass., 1992.

[GHK94] A. J. Grove, J. Y. Halpern, and D. Koller. Random worlds and maximum entropy. Journal of A.I. Research, 2:33-88, 1994. 
[GHK96a] A. J. Grove, J. Y. Halpern, and D. Koller. Asymptotic conditional probabilities: the unary case. SIAM Journal on Computing, 25(1):1-51, 1996.

[GHK96b] A. J. Grove, J. Y. Halpern, and D. Koller. Asymptotic conditional probabilities: the non-unary case. Journal of Symbolic Logic, 61(1):250-275, 1996.

[GMP90] M. Goldszmidt, P. Morris, and J. Pearl. A maximum entropy approach to nonmonotonic reasoning. In Proc. Eighth National Conference on Artificial Intelligence (AAAI'90), pages 646-652, 1990.

[GMP93] M. Goldszmidt, P. Morris, and J. Pearl. A maximum entropy approach to nonmonotonic reasoning. IEEE Transactions of Pattern Analysis and Machine Intelligence, 15(3):220-232, 1993.

[Gol87] S. A. Goldman. Efficient methods for calculating maximum entropy distributions. Master's thesis, MIT EECS Department, 1987.

[Goo92] S. D. Goodwin. Second order direct inference: A reference class selection policy. International Journal of Expert Systems: Research and Applications, 5(3):185-210, 1992.

[GP90] H. Geffner and J. Pearl. A framework for reasoning with defaults. In H. E. Kyburg, Jr., R. Loui, and G. Carlson, editors, Knowledge Representation and Defeasible Reasoning, pages 245-26. Kluwer Academic Press, Dordrecht, Netherlands, 1990.

[GP92] H. Geffner and J. Pearl. Conditional entailment: bridging two approaches to default reasoning. Artificial Intelligence, 53(2-3):209-244, 1992.

[Hac75] I. Hacking. The Emergence of Probability. Cambridge University Press, Cambridge, U.K., 1975.

[Hal90] J. Y. Halpern. An analysis of first-order logics of probability. Artificial Intelligence, 46:311-350, 1990.

[HK95] J. Y. Halpern and D. Koller. Representation dependence in probabilistic inference. In Proc. Fourteenth International Joint Conference on Artificial Intelligence (IJCAI '95), pages 1853-1860, 1995.

[HM87] S. Hanks and S. McDermott. Nonmonotonic logic and temporal projection. Artificial Intelligence, 33(3):379-412, 1987.

[Hun89] D. Hunter. Causality and maximum entropy updating. International Journal of Approximate Reasoning, 3(1):379-406, 1989.

[Jay78] E. T. Jaynes. Where do we stand on maximum entropy? In R. D. Levine and M. Tribus, editors, The Maximum Entropy Formalism, pages 15-118. MIT Press, Cambridge, Mass., 1978.

[Jef68] R. C. Jeffrey. Probable knowledge. In I. Lakatos, editor, International Colloquium in the Philosophy of Science: The Problem of Inductive Logic, pages 157-185. North-Holland, Amsterdam, 1968. 
[Joh32] W. E. Johnson. Probability: The deductive and inductive problems. Mind, 41(164):409-423, 1932.

[KH92] D. Koller and J. Y. Halpern. A logic for approximate reasoning. In Proc. Third International Conference on Principles of Knowledge Representation and Reasoning (KR '92), pages 153-164. 1992.

[KH96] D. Koller and J.Y. Halpern. Irrelevance and conditioning in first-order probabilistic logic. In Proc. Thirteenth National Conference on Artificial Intelligence (AAAI '96), pages 569-576, 1996.

[KLM90] S. Kraus, D. Lehmann, and M. Magidor. Nonmonotonic reasoning, preferential models and cumulative logics. Artificial Intelligence, 44:167-207, 1990.

[Kyb61] H. E Kyburg, Jr. Probability and the Logic of Rational Belief. Wesleyan University Press, Middletown, Connecticut, 1961.

[Kyb74] H. E. Kyburg, Jr. The Logical Foundations of Statistical Inference. Reidel, Dordrecht, Netherlands, 1974.

[Kyb83] H. E. Kyburg, Jr. The reference class. Philosophy of Science, 50(3):374-397, 1983.

[Kyb88] H. E. Kyburg, Jr. Full beliefs. Theory and Decision, 25:137-162, 1988.

[Lan80] L. D. Landau. Statistical Physics, volume 1. Pergamon Press, 1980.

[Lif89] V. Lifschitz. Benchmark problems for formal non-monotonic reasoning, version 2.00. In M. Reinfrank, J. de Kleer, M. L. Ginsberg, and E. Sandewall, editors, NonMonotonic Reasoning: 2nd International Workshop (Lecture Notes in Artificial Intelligence 346), pages 202-219. Springer-Verlag, 1989.

[LM81] R. J. Larsen and M. L. Mark. An introduction to mathematical statistics and its applications. Prentice-Hall, Englewood Cliffs, NJ, 1981.

[LM90] D. Lehmann and M. Magidor. Preferential logics: the predicate calculus case. In R. Parikh, editor, Theoretical Aspects of Reasoning about Knowledge: Proc. Third Conference, pages 57-72, San Francisco, 1990. Morgan Kaufmann.

[LM92] D. Lehmann and M. Magidor. What does a conditional knowledge base entail? Artificial Intelligence, 55(1):1-60, 1992.

[LR57] R. D. Luce and H. Raiffa. Games and Decisions. Wiley, New York, 1957.

[Mak89] D. Makinson. General theory of cumulative inference. In M. Reinfrank, J. de Kleer, M. L. Ginsberg, and E. Sandewall, editors, Non-Monotonic Reasoning: 2nd International Workshop (Lecture Notes in Artificial Intelligence 346), pages 1-18. Springer-Verlag, 1989.

[Mak94] D. Makinson. General patterns in nonmonotonic reasoning. In D. Gabbay, C. Hogger, and J. Robinson, editors, Handbook of Logic in Artificial Intelligence and Logic Programming, volume 3, pages 35-110. Oxford University Press, 1994. 
[McC80] J. McCarthy. Circumscription - a form of non-monotonic reasoning. Artificial Intelligence, 13:27-39, 1980.

[McC86] J. McCarthy. Applications of circumscription to formalizing common-sense knowledge. Artificial Intelligence, 28:86-116, 1986.

[MH69] J. McCarthy and P. J. Hayes. Some philosophical problems from the standpoint of artificial intelligence. In D. Michie, editor, Machine Intelligence 4, pages 463-502. Edinburgh University Press, Edinburgh, 1969.

[Moo85] R. C. Moore. Semantical considerations on nonmonotonic logic. Artificial Intelligence, 25:75-94, 1985.

[Mor93] M. Morreau. The conditional logic of generalizations. In Proceedings of the IJCAI Workshop on Conditionals in Knowledge Representation, pages 108-118, 1993.

[Pea88] J. Pearl. Probabilistic Reasoning in Intelligent Systems. Morgan Kaufmann, San Francisco, 1988.

[Pea89] J. Pearl. Probabilistic semantics for nonmonotonic reasoning: a survey. In Proc. First International Conference on Principles of Knowledge Representation and Reasoning (KR '89), pages 505-516, 1989. Reprinted in G. Shafer and J. Pearl (Eds.), Readings in Uncertain Reasoning, pp. 699-710. San Francisco: Morgan Kaufmann, 1990.

[Pea90] J. Pearl. System Z: A natural ordering of defaults with tractable applications to nonmonotonic reasoning. In Theoretical Aspects of Reasoning about Knowledge: Proc. Third Conference, pages 121-135. 1990.

[Pol90] J. L. Pollock. Nomic Probabilities and the Foundations of Induction. Oxford University Press, Oxford, U.K., 1990.

[Poo89] D. Poole. What the lottery paradox tells us about default reasoning. In Proc. First International Conference on Principles of Knowledge Representation and Reasoning (KR '89), pages 333-340. 1989.

[Poo91] D. Poole. The effect of knowledge on belief: conditioning, specificity and the lottery paradox in default reasoning. Artificial Intelligence, 49(1-3):282-307, 1991.

[PV89] J. B. Paris and A. Vencovska. On the applicability of maximum entropy to inexact reasoning. International Journal of Approximate Reasoning, 3:1-34, 1989.

[RC81] R. Reiter and G. Criscuolo. On interacting defaults. In Proc. Seventh International Joint Conference on Artificial Intelligence (IJCAI' '81), pages 270-276, 1981.

[Rei49] H. Reichenbach. The Theory of Probability. University of California Press, Berkeley, 1949. Translation and revision of German edition, published as Wahrscheinlichkeitslehre, 1935.

[Rei80] R. Reiter. A logic for default reasoning. Artificial Intelligence, 13:81-132, 1980. 
[Sav54] L. J. Savage. Foundations of Statistics. Wiley, New York, 1954.

[Sha76] G. Shafer. A Mathematical Theory of Evidence. Princeton University Press, Princeton, N.J., 1976.

[Sha89] L. Shastri. Default reasoning in semantic networks: a formalization of recognition and inheritance. Artificial Intelligence, 39(3):285-355, 1989.

[Sha93] G. Shafer. Personal communication, 1993.

[SW49] C. Shannon and W. Weaver. The Mathematical Theory of Communication. University of Illinois Press, Urbana-Champaign, Ill., 1949.

[THT87] D. S. Touretzky, J. F. Horty, and R. H. Thomason. A clash of intuitions: the current state of nonmonotonic multiple inheritance systems. In Proc. Tenth International Joint Conference on Artificial Intelligence (IJCAI' 87 ), pages 476-482, 1987. 